\title{
Novel Engineered Nanostructured Complex Oxide for High Temperature Thermoelectric Power Generation
}

Diego F. Palacio

West Virginia University

Follow this and additional works at: https://researchrepository.wvu.edu/etd

\section{Recommended Citation}

Palacio, Diego F., "Novel Engineered Nanostructured Complex Oxide for High Temperature Thermoelectric Power Generation" (2012). Graduate Theses, Dissertations, and Problem Reports. 198.

https://researchrepository.wvu.edu/etd/198

This Thesis is protected by copyright and/or related rights. It has been brought to you by the The Research Repository @ WVU with permission from the rights-holder(s). You are free to use this Thesis in any way that is permitted by the copyright and related rights legislation that applies to your use. For other uses you must obtain permission from the rights-holder(s) directly, unless additional rights are indicated by a Creative Commons license in the record and/ or on the work itself. This Thesis has been accepted for inclusion in WVU Graduate Theses, Dissertations, and Problem Reports collection by an authorized administrator of The Research Repository @ WVU. For more information, please contact researchrepository@mail.wvu.edu. 


\title{
Novel Engineered Nanostructured Complex Oxide for High Temperature Thermoelectric Power Generation
}

\author{
Diego F. Palacio \\ Thesis submitted to the \\ Benjamin M. Statler College of Engineering and Mineral Resources \\ at West Virginia University \\ in partial fulfillment of the requirements \\ for the degree of \\ Master of Science \\ in \\ Aerospace Engineering \\ Xueyan Song, Ph.D., Chair \\ Daneesh Simien, Ph.D. \\ Ever J. Barbero, Ph.D. \\ Department of Mechanical and Aerospace Engineering \\ Morgantown, West Virginia \\ 2012
}

Keywords: Thermoelectricity; Layered Calcium Cobalt Oxide; Nanoinclusions; Power Factor Copyright 2012 Diego F. Palacio 


\section{Abstract \\ Novel Engineered Nanostructured Complex Oxide for High Temperature Thermoelectric Power Generation}

\section{Diego F. Palacio}

Thermoelectric power generators are a promising technology that can produce electrical power directly from waste heat emitted in energy production and consumption systems. The essential component of thermoelectric generators is the thermoelectric material which performs the energy conversion process. In order to make thermoelectric devices to be considered a significant contributor to US energy infrastructure, it is essential first to foster improvements in high performance thermoelectric materials. They must possess not only a high conversion efficiency, but also need be composed of nontoxic and abundantly available elemental materials having high chemical stability in air up to 800-1000 K. In comparison with the conventional thermoelectric materials (intermetallic compounds); the layered oxide materials, such as the newly developed nontoxic p-type $\mathrm{Ca}_{3} \mathrm{Co}_{4} \mathrm{O}_{9}$, are particularly promising for thermoelectric applications because of their high stability at high temperatures in air.

In recent years, the layered cobalt oxide $\mathrm{Ca}_{3} \mathrm{Co}_{4} \mathrm{O}_{9}$ system has gained great attention due to its outstanding thermoelectric performance $(Z T=0.83$ at $973 \mathrm{~K})$ for the single crystal. Single crystals, however, are too small and expensive for practical applications. The current challenge for developing oxide thermoelectric materials is to improve the conversion efficiency of the polycrystalline $\mathrm{Ca}_{3} \mathrm{Co}_{4} \mathrm{O}_{9}$ ceramic, which is currently lower than that of the conventional thermoelectric materials. The distinctive objective in this research is to optimize the energy interconversion properties of p-type $\mathrm{Ca}_{3} \mathrm{Co}_{4} \mathrm{O}_{9}$ ceramic, through nano-structure engineering approaches. Novel methods of introducing the appropriate dopants and other nano-structures into the oxide were investigated, and the effect of dopants and nano-structure engineering on the thermoelectric performance of the $\mathrm{Ca}_{3} \mathrm{Co}_{4} \mathrm{O}_{9}$ system was evaluated. The above study will be instrumental for fabricating polycrystalline $\mathrm{Ca}_{3} \mathrm{Co}_{4} \mathrm{O}_{9}$ with optimized microstructure and enhanced thermoelectric properties. 
To my dearly loved parents and brothers... 


\section{Acknowledgements}

I want to express my deep and sincere gratitude to my research advisor Dr. Xueyan Song for her contribution to my professional development by giving me the opportunity to work with her as research assistant; it has been a privilege and the most valuable experience. I'll be always grateful for all her support, guidance, motivation, patience and generosity.

I want to especially thank Dr. Yun Chen for all his guidance during the last years and for contributing with his expertise to the development of this research work.

I have also to express my sincere gratitude to Maria Torres for all her hard work in the preparation of the ceramic samples, measurement of the thermoelectric properties and SEM imaging. Completion of this thesis work wouldn't be possible without her help.

Thanks also to Dr. Xuezhang Xiao for his contribution in the measurement of the thermoelectric properties of the experimental samples.

Especial thanks to the other members of my research group: Song Chen for his assistance and expertise in the TEM operation and sample preparation, and Xueqin Chen for her assistance with the SEM imaging. Working with them has been a real pleasure.

I also want to thank the advisory committee members Dr. Daneesh Simien and Dr. Ever J. Barbero for their valuable time and support during the evaluation of this thesis work.

Finally, thanks to the Benjamin M. Statler College of Engineering and Mineral Resources at WVU, especially to the Department of Mechanical and Aerospace Engineering. Thanks to all my professors. 


\section{Table of Contents}

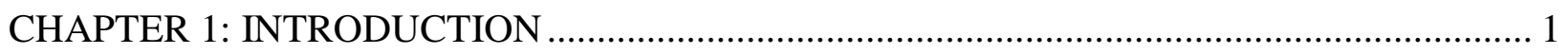

1.1 Background-Statement of the Problem ................................................................ 1

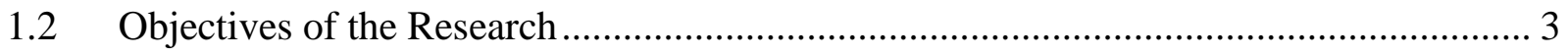

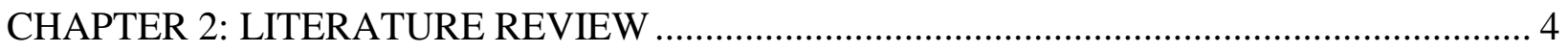

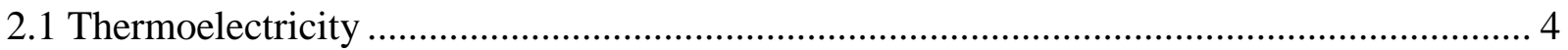

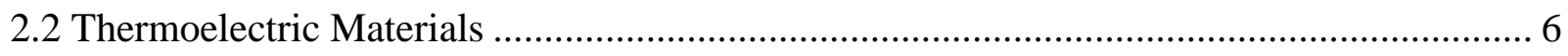

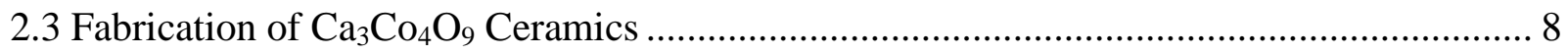

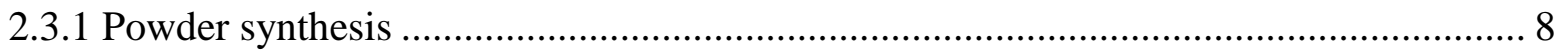

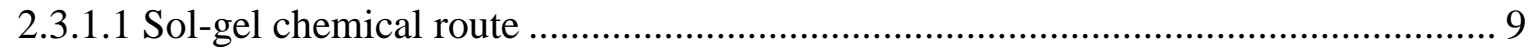

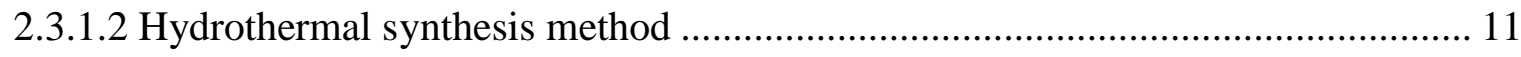

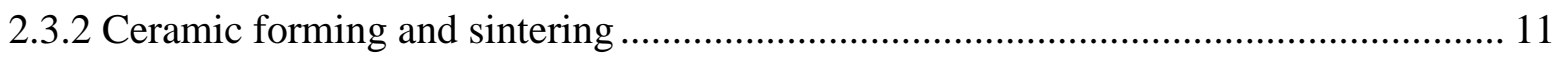

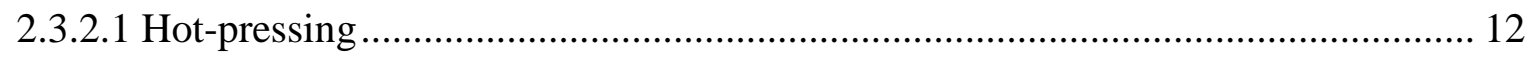

2.3.2.2 Spark plasma sintering ...................................................................... 14

2.4 Thermoelectric Properties Improvement .......................................................... 14

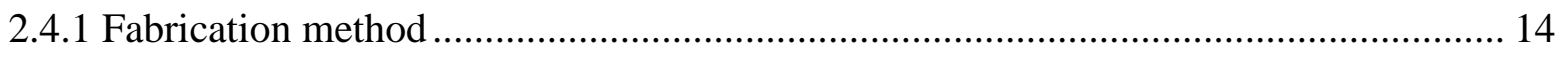

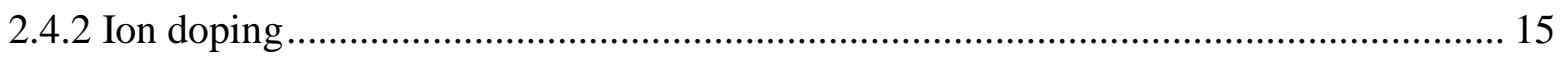

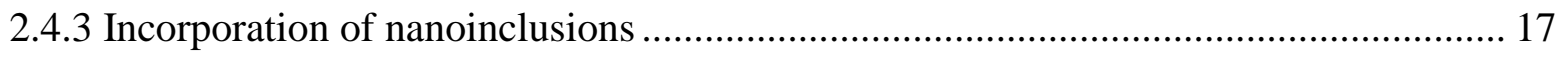

CHAPTER 3: EFFECT OF THE SOL-GEL SOLVENT ON THE THERMOELECTRIC PROPERTIES OF PURE $\mathrm{Ca}_{3} \mathrm{Co}_{4} \mathrm{O}_{9}$ CERAMICS ......................................................... 21 
3.1 Experimental Procedure

3.2 Results and Discussion

23

CHAPTER 4: EFFECT OF THE ADDITION OF OXIDE CERAMICS ON THE THERMOELECTRIC PROPERTIES OF $\mathrm{Ca}_{3} \mathrm{Co}_{4} \mathrm{O}_{9}$ CERAMICS …………………….............. 31

4.1 Effect of YSZ Addition on the Thermoelectric Properties of $\mathrm{Ca}_{3} \mathrm{Co}_{4} \mathrm{O}_{9}$ Ceramics ............. 31

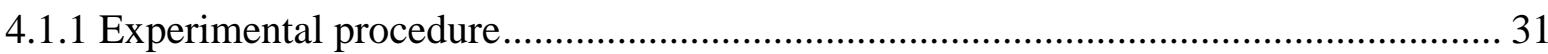

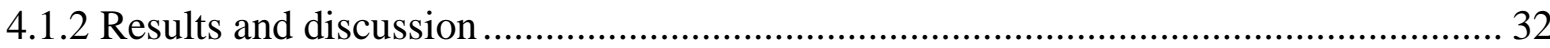

4.2 Effect of $\mathrm{CoAl}_{2} \mathrm{O}_{4}$ Addition on the Thermoelectric Properties of $\mathrm{Ca}_{3} \mathrm{Co}_{4} \mathrm{O}_{9}$ Ceramics ..... 38

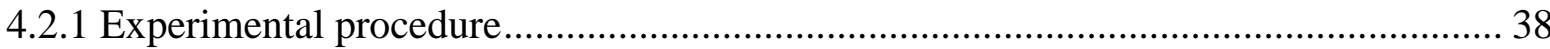

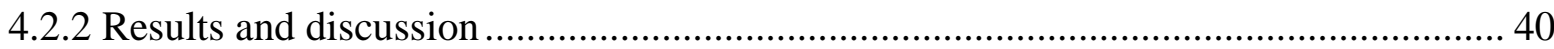

4.3 Effect of $\mathrm{NiFe}_{2} \mathrm{O}_{4}$ Addition on the Thermoelectric Properties of $\mathrm{Ca}_{3} \mathrm{Co}_{4} \mathrm{O}_{9}$ Ceramics ...... 46

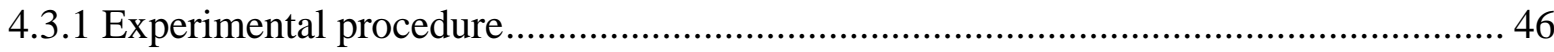

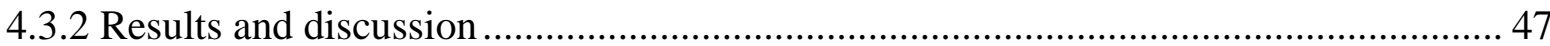

CHAPTER 5: EFFECT OF THE ADDITION OF PRECIOUS METALS ON THE THERMOELECTRIC PROPERTIES OF $\mathrm{Ca}_{3} \mathrm{Co}_{4} \mathrm{O}_{9}$ CERAMICS …......................................... 54

5.1 Effect of Pd doping on the thermoelectric properties of $\mathrm{Ca}_{3} \mathrm{Co}_{4} \mathrm{O}_{9}$ Ceramics .................... 54

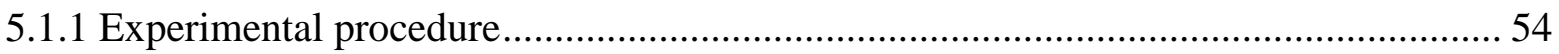

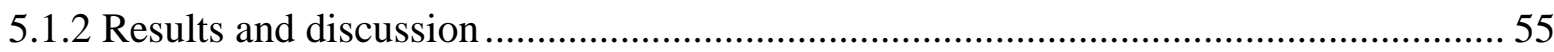

5.2 Effect of Au Addition on the Thermoelectric Properties of $\mathrm{Ca}_{3} \mathrm{Co}_{4} \mathrm{O}_{9}$ Ceramics............... 62 


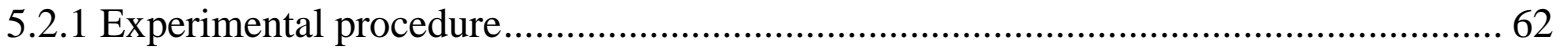

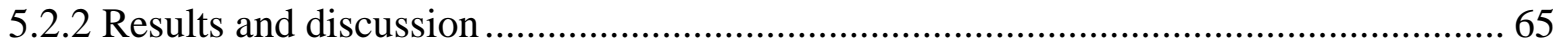

CHAPTER 6: CONCLUSIONS AND FUTURE WORK .................................................... 72

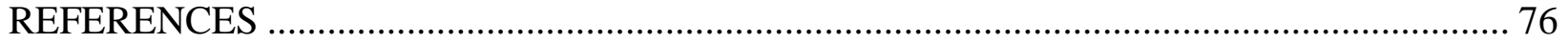




\section{List of Figures}

Figure 1. Carrier concentration dependence of $S, \rho$ and $\kappa$. Source: Ohtaki [4].

Figure 2. Dimensionless figures-of-merit for the $\mathrm{Bi}_{2} \mathrm{Te}_{3}, \mathrm{PbTe}$ and $\mathrm{Si}_{1-\mathrm{x}} \mathrm{Ge}_{\mathrm{x}}$ thermoelectric

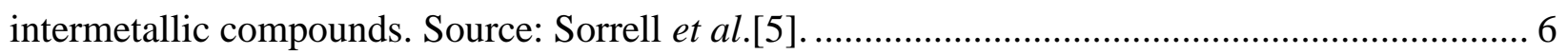

Figure 3. Layered crystal structures of different metal oxide systems: $\mathrm{NaCo}_{2} \mathrm{O}_{4}, \mathrm{Ca}_{3} \mathrm{Co}_{4} \mathrm{O}_{9}$ and

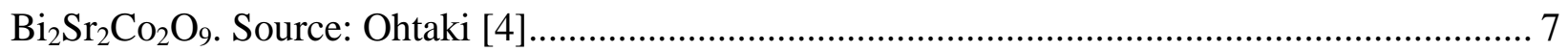

Figure 4. Seebeck coefficient, electrical resistivity and dimensionless figure-of-merit for the single crystal CCO. Source: Shikano and Funahashi [6]........................................................ 8

Figure 5. Schematic view of the hot-pressing process during powder compaction. Figure by Groza, J.R.[44]. 13

Figure 6. Improvement of the thermopower and power factor of $\mathrm{CCO}$ by doping late rare earths into the CCO. Source: Nong et al.[12]. 16

Figure 7. Flow diagram of the fabrication route of the three pure CCO ceramics synthesized: DI water CCO, EG-50\% CCO and DEG CCO. The fabrication routes differ in the solvent employed. 22

Figure 8. SEM micrographs of the synthesizes pure CCO powder using different solvents (a) DI water (b) EG-50\% and (c) DEG... 23

Figure 9. SEM micrographs from the pressed plane and cross-sectional fractured surface of the DI water CCO (a) and (b), EG-50\% CCO (c) and (d), and DEG CCO (e) and (f). The arrows indicate the direction of the applied pressure. 25

Figure 10. Nanostructure of the DI water CCO: (a) CCO grain composed by nano-lamellas, (b) nano-lamellas made up by crystalline planes and (c) electron diffraction pattern from the grain.26 
Figure 11. Nanostructure of the EG-50\% CCO: (a) CCO grain composed by nano-lamellas, (b) bundle of nano-lamellas and (c) its corresponding electron diffraction pattern. .......................... 26

Figure 12. Nanostructure of the DEG CCO: (a) CCO grains composed by nano-lamellas, (b) bundle of nano-lamellas and (c) and electron diffraction pattern............................................... 27 Figure 13. TEM micrographs of cobalt oxide grains with their respective electron diffraction patterns from: (a) and (b) DI water CCO sample, (c) and (d) EG-50\% CCO sample, and (e) and

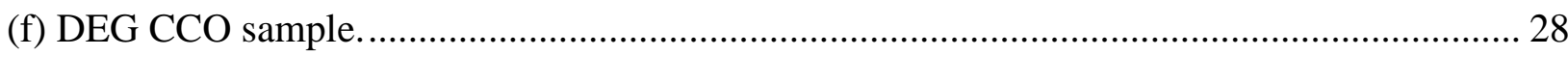

Figure 14. Seebeck coefficient, resistivity and power factor curves of the three experimental CCO samples. 30

Figure 15. Flow diagram of the fabrication route of the CCO/YSZ-2 wt $\%$ ceramic composite. . 31 Figure 16. SEM micrograph of the synthesized CCO/YSZ ceramic powder before consolidation. 32

Figure 17. SEM micrographs of the morphology of the CCO/YSZ composite showing: (a) the pressed plane and (b) the fractured cross-section. The arrow indicates the applied stress direction.

Figure 18. TEM micrographs from Ca3Co4O9/YSZ ceramic: (a) polycrystalline region and (b) HRTEM image of the atomic planes. 34

Figure 19. TEM micrographs showing ceramic regions affected by penetration of YSZ into the CCO grains. 35

Figure 20. Seebeck coefficient, resistivity and power factor of the CCO/YSZ-2 wt\% composite compared to the values for the pure $\mathrm{CCO}$ ceramic system. 38

Figure 21. Temperature profile as a function of time applied during hydrothermal synthesis of the $\mathrm{CoAl}_{2} \mathrm{O}_{4}$ nanopowder. 39 
Figure 22. Flow diagram of the fabrication route of the $\mathrm{CCO} / \mathrm{CoAl}_{2} \mathrm{O}_{4}-10 \mathrm{wt} \%$ composite ceramic. 40

Figure 23. TEM micrographs of the hydrothermal $\mathrm{CoAl}_{2} \mathrm{O}_{4}$ nanopowder synthesized: (a) bright field image and (b) selected area electron diffraction pattern. 41 Figure 24. SEM micrographs of the $\mathrm{CCO} / \mathrm{CoAl}_{2} \mathrm{O}_{4}$ composite ceramic showing the morphology of: (a) the pressed plane and (b) a fractured cross-section surface. The arrow indicates the applied stress direction.

Figure 25. TEM micrographs showing: (a) the layered structure of the $\mathrm{CCO}$ and (b) $\mathrm{a} \mathrm{Co}_{3} \mathrm{O}_{4}$ grain with its respective diffraction pattern (c). 43

Figure 26. TEM micrographs showing the amorphous Al-O phase found within the CCO grains.

Figure 27. Seebeck coefficient, resistivity and power factor of the experimental $\mathrm{CCO} / \mathrm{CoAl}_{2} \mathrm{O}_{4}$ alloy compared with the baseline. 46

Figure 28. Flow diagram of the fabrication route of the $\mathrm{Ca}_{3} \mathrm{Co}_{4} \mathrm{O}_{9} / \mathrm{NiFe}_{2} \mathrm{O}_{4}-2$ wt $\%$ composite ceramic.

Figure 29. SEM micrographs of the $\mathrm{CCO} / \mathrm{NiFe}_{2} \mathrm{O}_{4}-2$ wt $\%$ composite ceramic showing the morphology of: (a) the pressed plane and (b) a fractured cross-section surface. The arrow indicates the applied stress direction. 48 Figure 30. TEM micrographs from the $\mathrm{CCO} / \mathrm{NiFe}_{2} \mathrm{O}_{4}$ composite: (a) bright field image, (b) HRTEM image showing the atomic planes and nano-lamellas and (c) its respective electron diffraction pattern. The direction of the $c$-axis is indicated.

Figure 31. TEM micrographs and diffraction patterns from: (a) and (c) the $\mathrm{Co}_{3} \mathrm{O}_{4}$ phase, and (b) and (d) the $\mathrm{Co}_{0.5} \mathrm{Ni}_{0.5} \mathrm{O}$ phase. 50 
Figure 32. (a) $\mathrm{TEM}$ micrograph from a $\mathrm{Co}_{2} \mathrm{NiO}_{4}$ grain located within the $\mathrm{CCO}$ and its respective electron diffraction pattern (b) corresponding to the [011] zone axis. 51

Figure 33. Seebeck coefficient, resistivity and power factor curves of the $\mathrm{Ca}_{3} \mathrm{Co}_{4} \mathrm{O}_{9} / \mathrm{NiFe}_{2} \mathrm{O}_{4}-2$ wt $\%$ composite compared to the baseline data. 53

Figure 34. Fabrication route for the $\mathrm{Ca}_{3} \mathrm{Co}_{4-\mathrm{x}} \mathrm{Pd}_{\mathrm{x}} \mathrm{O}_{9}(\mathrm{x}=0,0.01$ and 0.05$)$ ceramics. 54

Figure 35. SEM micrographs of the $\mathrm{x}=0.01$ ceramic system showing two surfaces: (a) the pressed plane and (b) a fractured cross-section. The arrow indicates the applied stress direction. 56 Figure 36. SEM micrographs of the $\mathrm{x}=0.05$ ceramic system showing two surfaces: (a) the pressed plane and (b) a fractured cross-section. The arrow indicates the applied stress direction. 56 Figure 37. TEM bright field images (a) and (b) of nanoinclusions within ceramic grains of the $\mathrm{x}=0.01$ system. (c) HRTEM image of the grain boundary between a nanoparticle (left) and the CCO substrate (right) 57

Figure 38. TEM bright field images (a) and (b) of the nanoinclusions within the ceramic grains of the $\mathrm{x}=0.05$ system. (c) HRTEM image of the grain boundary between a nanoparticle (right) and the CCO substrate (left). 57 Figure 39. (a) $\mathrm{TEM}$ micrograph from the $\mathrm{x}=0.01$ system showing a $\mathrm{Pd}_{3.5} \mathrm{O}_{4}$ nanoparticle on the $\mathrm{CCO}$, (b) and (c) show the electron diffraction patterns from the $\mathrm{CCO}$ substrate and the $\mathrm{Pd}_{3.5} \mathrm{O}_{4}$ nanoinclusion respectively. 59 Figure 40. TEM micrograph showing a $\mathrm{Pd}_{3.5} \mathrm{O}_{4}$ nanoinclusion embedded within the $\mathrm{CCO}$ grain the $\mathrm{x}=0.01$ ceramic system. 59 Figure 41. TEM micrograph and two electron diffraction patterns from a nanoparticle of the $\mathrm{x}=0.05$ ceramic. The diffraction patterns were indexed as the [100] and [111] zone axes of the cubic $\mathrm{Pd}_{3.5} \mathrm{O}_{4}$ phase. 60 
Figure 42. Seebeck coefficient, electrical resistivity and power factor of the experimental $\mathrm{CCO} / \mathrm{Pd}$ systems $(\mathrm{x}=0,0.01$ and 0.05$)$.

Figure 43. Temperature profile as a function of time applied during hydrothermal Au deposition.

Figure 44. Flow diagram of the fabrication route of the CCO/Au-10 wt\% composite ceramic... 64

Figure 45. TEM micrograph of the hydrothermally synthesized CCO/Au powder. 65

Figure 46. SEM micrograph of the prepared $\mathrm{CCO} / \mathrm{Au}$ composite ceramic showing $\mathrm{Au}$ nanoparticles uniformly distributed all over the CCO. 66

Figure 47. SEM micrographs from: (a) the pressed plane and (b) the fractured cross-section of the $\mathrm{CCO} / \mathrm{Au}$ pellet. The arrow indicates the direction of the applied pressing. 66 Figure 48. TEM micrographs from the nanostructure of the $\mathrm{CCO} / \mathrm{Au}$ ceramic system. $\mathrm{Au}$ nanoparticles can be observed all over the CCO grains.

Figure 49. (a) TEM micrograph of an $\mathrm{Au}$ nanoinclusion embedded within the layered nanostructure of the $\mathrm{CCO}$, (b) and (c) electron diffraction patterns of the CCO substrate and the Au nanoparticle respectively. 68 Figure 50. Bright field TEM micrographs of Au nanoparticles located (a) within the CCO and (b) at the grain boundary. 69

Figure 51. Seebeck coefficient, resistivity and power factor of the $\mathrm{CCO} / \mathrm{Au}-10 \mathrm{wt} \%$ system compared with the baseline data. 71 


\section{List of Tables}

Table 1. Representative EDS data from the polycrystalline region of the CCO/YSZ ceramic.... 34

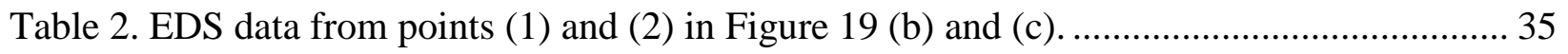

Table 3. Representative EDS data from the $\mathrm{CoAl}_{2} \mathrm{O}_{4}$ nanoparticles. .................................... 41

Table 4. Representative EDS data from the CCO and cobalt oxide grain presented in Figure 25

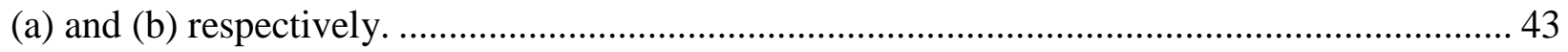

Table 5. Representative EDS data from the Al-O amorphous phase imaged in Figure 26. ........ 44

Table 6. Representative EDS data from the $\mathrm{CCO} / \mathrm{NiFe}_{2} \mathrm{O}_{4}$ ceramic. ..................................... 49

Table 7. EDS data from the grains presented in Figure 31 (a) and (b)................................. 50

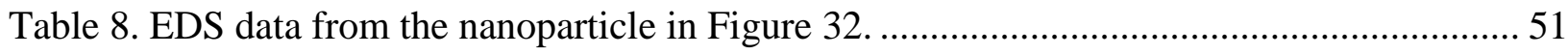

Table 9. Representative EDS data from the ceramic grains and nanoparticles. ........................ 58 


\section{CHAPTER 1: INTRODUCTION}

\subsection{Background-Statement of the Problem}

Current society is supported by the massive consumption of energy; electricity and fossil fuels as coal, petroleum and natural gas are the main motors sustaining its accelerated development. Statistical projections reveal that the rates of energy consumption and carbon emissions will continue rising very fast leading to the imminent depletion of primary energy sources (as petroleum) and causing an environmental crisis. Consequently, different alternatives have been proposed to solve these problems, between them: to implement the use of renewable environmental-friendly energy sources and to improve the efficiency of the current energy consuming processes [1].

This research work intends to contribute to the improvement of the efficiency of the current energy consumption processes by the development of high-efficiency thermoelectric (TE) materials. TE materials are capable to produce electrical energy from heat and vice versa when implemented in a TE device. TE devices are an energy conversion technology known to be efficient and environmentally-friendly with potential to recover unexploited energy emitted as heat in energy production and consumption systems. It has been claimed that about $70 \%$ of the total energy employed in systems such as automobiles and factories is wasted in the form of heat [2]. Due to their reversible operation, simplicity (no moving parts required) and possibility to be employed in the nano-scale, TE devices possess many other applications of interest in the industry and technology sectors [3].

The heart of the TE device is the TE material which performs the energy conversion through the electrons. Thermoelectricity relates the thermal and electric phenomena in solids; the most 
important TE effects are the Seebeck effect and Peltier effect. The first one consists on inducing a voltage from a thermal gradient while the second one acts in the opposite way, producing a thermal gradient from an electric current. The conversion efficiency of a TE material is evaluated using the figure-of-merit $Z\left[K^{-1}\right]$ which is expressed as $Z=S^{2} / \rho \kappa$ where $S$ is the Seebeck coefficient, $\rho$ is the electrical resistivity and $\kappa$ is the thermal conductivity [4]. From this relation, thermoelectric materials with high $S$ and low $\rho$ and $\kappa$ are desired. The relation between $S^{2} / \rho$ (called the power factor) is usually employed as performance indicator in the preliminary stages of TE materials design.

TE materials are divided in two main categories: the conventional intermetallic compounds that are degenerate semiconductors (e.g., $\mathrm{Bi}_{2} \mathrm{Te}_{3}, \mathrm{PbTe}$ and $\mathrm{SiGe}$ ) and the recent metal oxides (e.g., $\mathrm{NaCo}_{2} \mathrm{O}_{4}$ and $\mathrm{Ca}_{3} \mathrm{Co}_{4} \mathrm{O}_{9}$ ) [5]. The layered metal oxides present significant advantages over the conventional TE materials such as chemical stability at high temperature in air, low cost and the use of non-toxic constituting elements. The misfit layered calcium cobaltite $\mathrm{Ca}_{3} \mathrm{Co}_{4} \mathrm{O}_{9}(\mathrm{CCO})$ system is studied in this research work due its high potential as p-type TE oxide for the fabrication of high efficiency TE devices operating at high temperature. TE performance of this material is known to be exceptional for the single crystal with $Z T=0.83$ at $973 \mathrm{~K}$ [6]; its applicability, however, is limited due to manufacturing difficulties and high cost. As a consequence, in the last years, many efforts have been made in the development of polycrystalline $\mathrm{CCO}$ ceramics with conversion efficiencies close to that of the single crystal. Improvement of the TE performance of the polycrystalline $\mathrm{CCO}$ has been reported to be effective by the single or combined action of chemistry modification (ion doping) [7-20], metallic nanoinclusions [21-26] and microstructure control (texturing) [27-35]. This study aims to determine the TE effect of adding different materials such as oxides and metals to the CCO. 


\subsection{Objectives of the Research}

The objective of this research work is to optimize the TE performance of the polycrystalline CCO ceramic by the addition of different alloying agents to modify its chemistry and to induce the formation of nanoinclusions within the $\mathrm{CCO}$ grains. For this purpose, two different kinds of materials will be tested as alloying agents: oxide ceramics (YSZ, $\mathrm{CoAl}_{2} \mathrm{O}_{4}$ and $\mathrm{NiFe}_{2} \mathrm{O}_{4}$ ) and precious metals $(\mathrm{Pd}$ and $\mathrm{Au})$.

The different ceramic systems will be synthesized using the sol-gel route and consolidated using hot-pressing followed by sintering. The addition of the alloying agents will vary between samples in order to test different synthesis and deposition mechanisms. The resultant systems will be examined using the SEM, TEM and EDS analytical techniques in order to determine whether doping and/or the formation of nanoinclusions occur.

Seebeck coefficient, electrical resistivity and power factor measurements will be performed for all the experimental systems and compared to a baseline. Pure CCO ceramics prepared under identical conditions will be employed as the baselines. Since this research work corresponds to a preliminary stage of TE materials design, thermal conductivity measurements are postponed for future work.

Additionally, the effect of the sol-gel solvent in the TE properties of pure CCO samples synthesized using the sol-gel route will be tested. Three different solvents are to be employed: $100 \%$ deionized water, $100 \%$ diethylene glycol and a mixture of deionized water and ethylene glycol (50-50 volume \%). The ceramics will be inspected using SEM and TEM in order to observe any micro/nano-structural variation of the CCO. Finally, the Seebeck coefficient, electrical resistivity and power factor of the samples will be measured and compared. 


\section{CHAPTER 2: LITERATURE REVIEW}

\subsection{Thermoelectricity}

Thermoelectricity is a physical phenomenon occurring at conducting solids which directly relates two of the most important phenomena studied by science and applied in engineering: thermodynamics and electricity. This fact explains the great interest thermoelectricity has generated in the scientific community since the German physicist Thomas Seebeck first observed it in 1821 [36]. Thermoelectricity has been traditionally employed for accurate temperature measurement with thermocouples and just from a few decades ago (due to the development of new and more efficient thermoelectric materials) this phenomenon has been applied for direct power generation.

Thermoelectricity in conducting solids is a reversible process that directly converts heat into electrical energy and vice versa by means of two fundamental and interrelated effects: the Seebeck effect and the Peltier effect. The conversion is performed by the electrons when migrate from one region of a solid to another due to a thermal gradient, producing an electric field and consequently an electric current.

The Seebeck effect consists in the generation of an electromotive force or voltage $(V)$ between two points of a conducting material that present a temperature gradient $(\Delta T)$. This relation can be expressed as $V=S \Delta T$ where $S$ is the Seebeck coefficient or thermopower. In contrast, the Peltier effect is considered, to some extent, the reverse process of the Seebeck effect. It consists in the reversible emission/absorption of heat $(Q)$ at the junctions of two TE materials when an electric (I) current passes through. The Peltier relation can be expressed as $Q=\Pi I$ where $\Pi$ is the Peltier coefficient. $S$ and $\Pi$ are related by the Onsager relation where $\Pi=S$ T [5]. Since the two 
thermoelectric effects are interrelated, measurement of only one of the coefficients is enough when studying the thermoelectric behavior of materials. The Seebeck coefficient has been traditionally used for this purpose.

The performance of a TE material is evaluated using the figure-of-merit $Z\left[K^{-1}\right]$ which is expressed as $Z=S^{2} / \rho \kappa$ where $S$ is the thermopower, $\rho$ is the electrical resistivity and $\kappa$ is the thermal conductivity. This relation is usually multiplied by the average operating temperature to obtain the dimensionless figure-of-merit $(Z T)$. According to the previous relation, TE materials with large $S$ along with low $\rho$ and $\kappa$ are sought [4]. The three parameters of $Z(S, \rho$ and $\kappa)$, however, are function of the carrier density $n$ as depicted in Figure 1. According to the figure, large thermopower is associated with low carrier concentration.

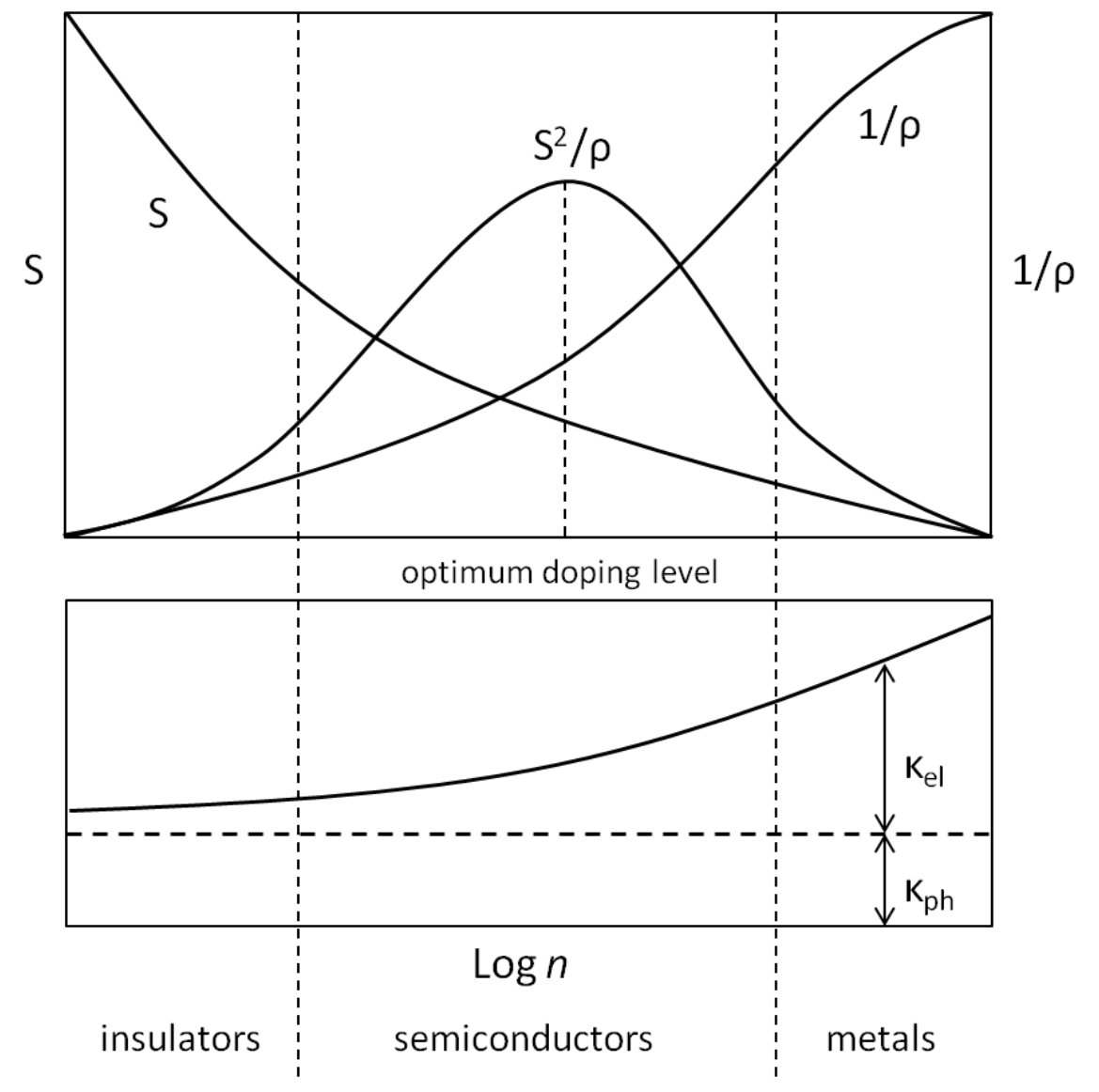

Figure 1. Carrier concentration dependence of $S, \rho$ and $\kappa$. Source: Ohtaki [4]. 


\subsection{Thermoelectric Materials}

TE materials possess the ability to directly convert a heat gradient into electricity and vice versa by action of the electrons under the TE effects. TE materials are classified in two main categories: the conventional intermetallic compounds and the recent metal oxides.

Conventional intermetallic compounds are binary systems that behave as high-mobility degenerate semiconductors which transport mechanism can be described using the conventional band theory [37]. The most widely used intermetallic compounds are $\mathrm{Bi}_{2} \mathrm{Te}_{3}, \mathrm{PbTe}$ and $\mathrm{SiGe}$; these thermoelectric materials have been successfully applied in thermoelectric devices [5]. Their dimensionless figures-of-merit $(Z T)$ are presented in Figure 2. Intermetallic compounds, however, present several disadvantages that limit their extended application like poor durability at high temperature and the use of volatile (e.g., Te), toxic, scarce and expensive precursor elements [22].

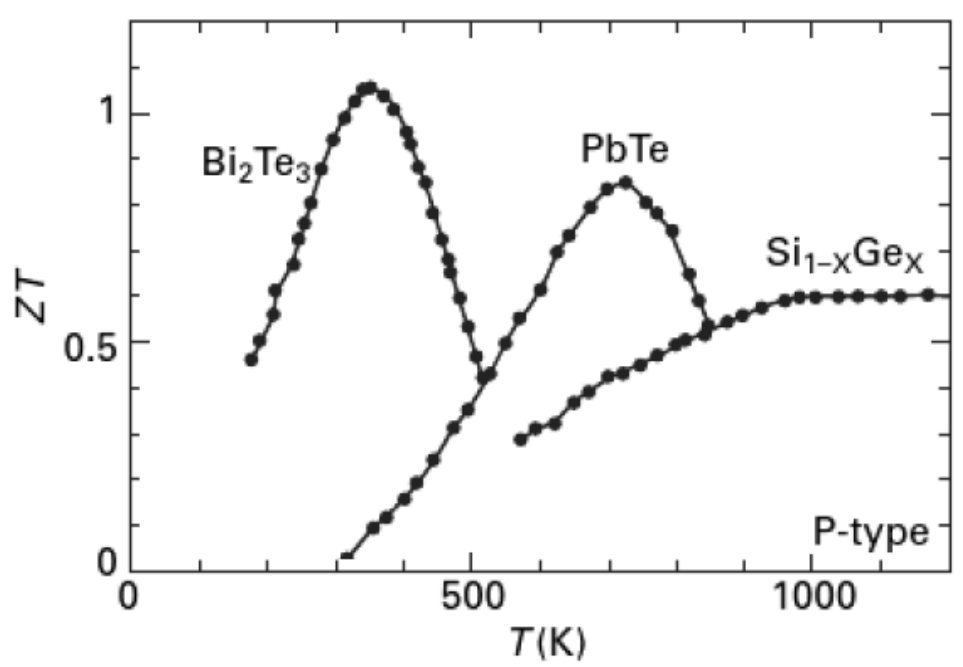

Figure 2. Dimensionless figures-of-merit for the $\mathrm{Bi}_{2} \mathrm{Te}_{3}, \mathrm{PbTe}$ and $\mathrm{Si}_{1-\mathrm{x}} \mathrm{Ge}_{\mathrm{x}}$ thermoelectric intermetallic compounds. Source: Sorrell et al.[5]. 
In contrast, metal oxide thermoelectric materials have been extensively studied since the discovery of the outstanding TE properties of the transition-metal oxide $\mathrm{NaCo}_{2} \mathrm{O}_{4}$ system by Terasaki in 1997 [38]; this material presents large thermopower coupled with low resistivity, which is unusual for metal oxides due to their low mobility. This exceptional TE performance is attributed to the layered nature of the $\mathrm{NaCo}_{2} \mathrm{O}_{4}$ system where $\mathrm{Na}$ ions and $\mathrm{CoO}_{2}$ layers are alternately stacked along the $c$-axis [37]. As a consequence the physical properties of this material are highly anisotropic. Other layered cobalt oxides with large TE performance and crystal structures related to that of $\mathrm{NaCo}_{2} \mathrm{O}_{4}$ have been also discovered in the last years; that is the case of the layered $\mathrm{Ca}_{3} \mathrm{Co}_{4} \mathrm{O}_{9}$ and $\mathrm{Bi}_{2} \mathrm{Sr}_{2} \mathrm{Co}_{2} \mathrm{O}_{9}$ systems that present the same hexagonal CdI2-type $\mathrm{CoO}_{2}$ layer [5]. Crystal structures of the mentioned layered metal oxide systems are presented in Figure 3. Metal oxide materials, contrary to the intermetallic compounds, are chemically stable at high temperature in air.
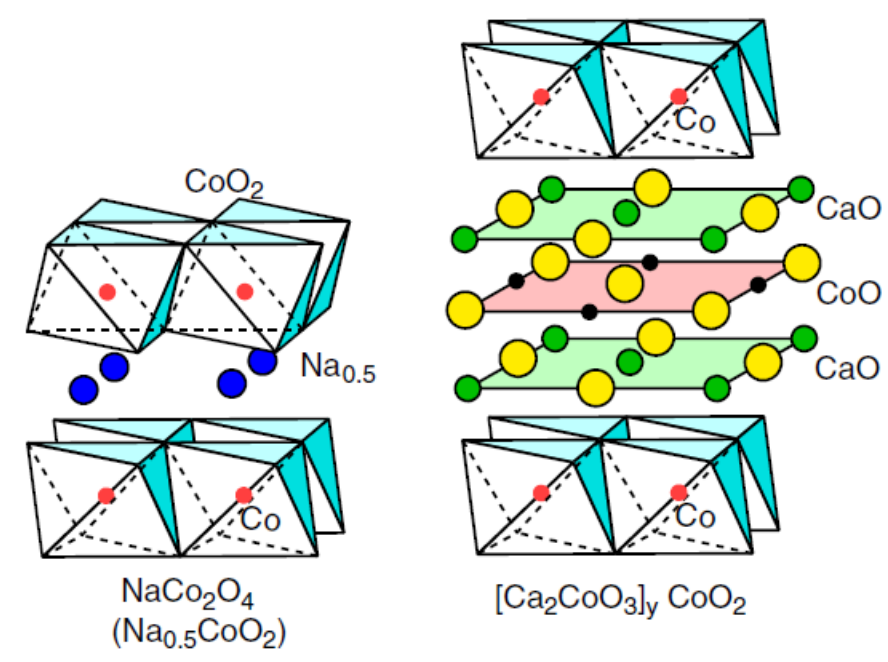

$$
\left[\mathrm{Ca}_{2} \mathrm{CoO}_{3}\right]_{\mathrm{y}} \mathrm{CoO}_{2}
$$

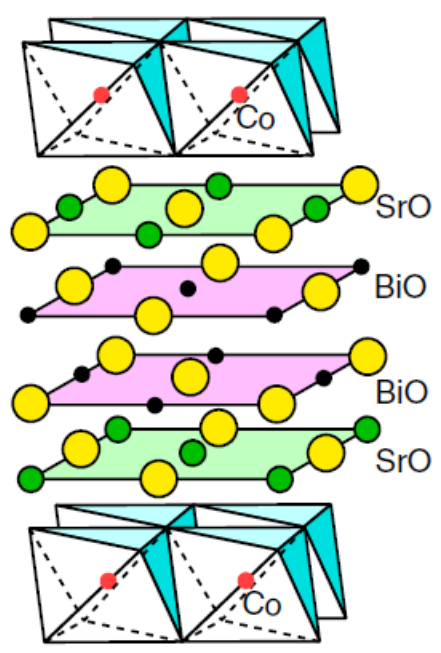

$$
\left[\mathrm{Bi}_{2} \mathrm{Sr}_{2} \mathrm{O}_{4}\right]_{y} \mathrm{CoO}_{2}
$$

Crystal structures of layered cobalt oxides.

Figure 3. Layered crystal structures of different metal oxide systems: $\mathrm{NaCo}_{2} \mathrm{O}_{4}, \mathrm{Ca}_{3} \mathrm{Co}_{4} \mathrm{O}_{9}$ and $\mathrm{Bi}_{2} \mathrm{Sr}_{2} \mathrm{Co}_{2} \mathrm{O}_{9}$. Source: Ohtaki [4]. 
The layered $\mathrm{Ca}_{3} \mathrm{Co}_{4} \mathrm{O}_{9}$ is one of the most promising $\mathrm{TE}$ materials for power generation applications at high temperature as demonstrated by Shikano and Funahashi [6]. They grew plate-like CCO single crystals using a modified strontium chloride flux technique and measured their thermoelectric properties along the $a b$-plane. It was shown that the $S-T$ curve of CCO presents metallic behavior, increasing monotonically with temperature. The positive values of $S$ in the whole measured temperature region indicates p-type conduction, meaning that the hole carrier is dominant. On the other hand, the $\rho$-T curve presented a waving behavior with broad peaks and bottoms, showing metal-like behavior below $600 \mathrm{~K}$ and semiconductor-like behavior above this temperature. These curves can be observed in Figure 4 along with the ZT-T curve. A $Z T$ of 0.83 at $973 \mathrm{~K}$ along the $a b$-plane was obtained.
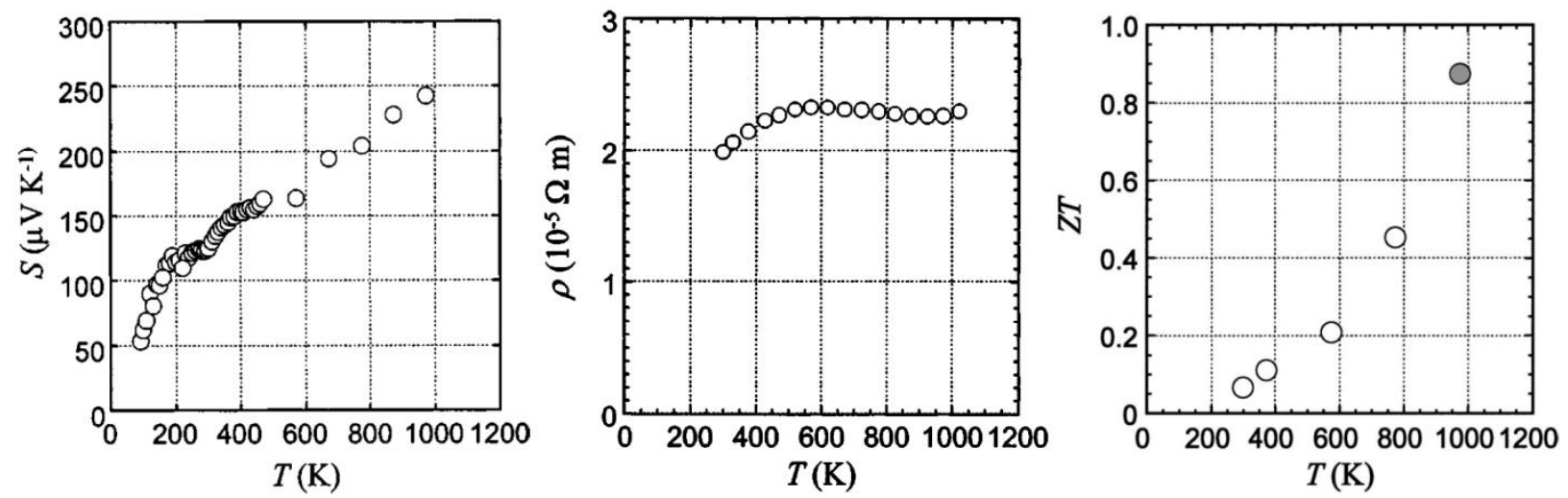

Figure 4. Seebeck coefficient, electrical resistivity and dimensionless figure-of-merit for the single crystal CCO. Source: Shikano and Funahashi [6].

\subsection{Fabrication of $\mathrm{Ca}_{3} \mathrm{Co}_{4} \mathrm{O}_{9}$ Ceramics}

\subsubsection{Powder synthesis}

It is well known that ceramic materials are highly sensitive to the preparation method employed for their synthesis [39]. Traditionally, ceramic powders have been synthesized using 
the solid-state reaction method which consists in the direct reaction of precursor powders at high temperature. This technique, however, presents some major drawbacks that limit its applicability such as difficulties to produce metastable phases and to control powder size and morphology (powder size over $10 \mu \mathrm{m}$ in diameter is usually obtained) [40]. Additionally, incomplete reaction and inhomogeneities typically occur. These drawbacks forced material science scientists to develop alternative methods for ceramic synthesis.

Solution synthesis methods such as sol-gel chemical route and hydrothermal synthesis are alternative methods for the synthesis of nanostructured ceramics. These methods present many advantages over the solid-state reaction method. The use of solutions as reaction media offer higher reactivity and the capability to produce fine and homogeneous ceramics with tailored properties. Ceramic properties such as size, chemical composition, morphology, etc., can be attained by controlling the precursor solution and process parameters. The sol-gel chemical route and the hydrothermal synthesis methods are discussed in more detail in the following sections.

\subsubsection{Sol-gel chemical route}

The sol-gel method is a wet chemical route used for the preparation of metal oxide and hydroxide particles [40]. It involves the hydrolysis, condensation and pyrolytic decomposition of the metal precursors producing fine and monodispersed metal oxides. This method allows (contrary to the traditional solid-state reaction method) to control the shape and morphology of the synthesized solid particles. Additionally, it can produce metastable phases as well as powders with superior homogeneity and purity while requiring lower temperature [41].

The Pechini method [41] or polymerizable complex (PC) method, a derivation of the sol-gel method, is a widely used process employed for ceramic powder synthesis due primarily to its 
implementation simplicity; neither highly specialized equipment nor inert atmosphere is required. Specifically, this method consists in the dissolution of precursor metal salts into a polymeric gel solvent: ethylene glycol (EG) containing citric acid (CA). Here, the role of the CA is to react with the metal salts to produce a metal-citrate complex and (after the temperature is increased to around $100-130^{\circ} \mathrm{C}$ ) to induce the polymerization of the solution by reacting with the EG. Due to the temperature increment, the viscosity of the solution increases drastically producing the "gelation" of the solution. It has been reported that optimal CA concentration in EG ranges from 50 to 60 mole \% for the preparation of complex oxides ever since viscosity of gel reaches its maximum into that range [41]. Peptization of the solution using nitric acid is also required to avoid particle agglomeration by counteracting the Van der Waals attractive forces through the generation of electrostatic repulsion forces between the suspended particles. A polymeric gel is obtained after elimination of excess EG which is treated at higher temperature $\left(450-600^{\circ} \mathrm{C}\right)$ to oxidize it and produce the metal oxide phases with the stoichiometry of the metal ions. As a result, fine and homogeneous complex oxide materials can be obtained by this route. Some residual carbonates, however, from the pyrolysis of the organic compounds could remain.

Alternatively the amorphous metal complex method [41] appears as a variant of the PC method. This method employs an aqueous precursor instead of a polymeric gel precursor, avoiding the formation of undesired carbonate impurities produced along with the metal oxide powder by reducing the amount of organic compounds employed for gelation. In this method EG solvent is replaced with water, this fact simplifies the process not only because of the safety and low cost of water but because a reduction of the processing temperature by almost $100^{\circ} \mathrm{C}$ can be obtained. The main difference with the PC method lies in the gelation process where an amorphous non-polymeric gel is formed. This method, however, presents several drawbacks 
limiting its application; for example, it can't be used with specific cations such as $\mathrm{Ti}$ and $\mathrm{Nb}$ that form unstable complexes with CA [41]. Additionally, the level of homogeneity of the powders obtained is inferior to that of the PC method.

\subsubsection{Hydrothermal synthesis method}

Hydrothermal method is a sophisticated technology for the preparation of fine engineered ceramic powders where the precursor materials react in an autoclave under the action of pressure and temperature. Hydrothermal synthesis offers direct crystallization of the ceramic materials from the aqueous solution at moderate temperature and pressure $\left(\mathrm{T}>25^{\circ} \mathrm{C}, \mathrm{P}>100 \mathrm{kPa}\right)$ [42]. This process can produce fine powders (much smaller than $1 \mu \mathrm{m}$ in size) with good homogeneity, narrow size distribution and weak agglomeration [43]. Additionally, specific powder features such as size, morphology, chemical composition, defect structure, etc., can be precisely controlled. The benefits of this method extend to powders production; it is also suitable to generate fibers, single crystals, films and even bulk ceramics with tailored properties. Although highly advanced, the hydrothermal synthesis is a very cost-effective technology (compared to the traditional ceramic synthesis methods) due to its time efficiency and low energy consumption (moderate temperature and pressure) for the crystallization of ceramic materials. In order to obtain the desired product; a prime quality solution is required. Pure and homogeneous precursors, along with specific additives are required for solution preparation; furthermore, precise control of $\mathrm{pH}$, solubility, chemical composition, particle dispersion, etc., must be assured.

\subsubsection{Ceramic forming and sintering}

It is known for the polycrystalline $\mathrm{CCO}$ ceramic that the better the grain alignment and packing density, the closer the TE properties will be to those of the single crystal ceramic. This 
means that the TE properties of the polycrystalline $\mathrm{Ca}_{3} \mathrm{Co}_{4} \mathrm{O}_{9}$ ceramic are highly dependent on the fabrication method employed to produce it. For ceramics in general, the fabrication method embraces all the processing steps performed to the synthesized ceramic powders with the purpose of getting a consolidated polycrystalline material. These steps include: powder dispersion, forming, drying, organic binder removal and sintering. Powder dispersion, when required, is accomplished by the use of dispersants such as polymers and other plasticizers agents. A variety of forming methods are available for bulk ceramic preparation, namely: dry and hot pressing, extrusion, casting methods, etc. The sintering is the heat treatment process performed as the last step of the ceramic fabrication; it transforms the green body into a dense polycrystalline product. During sintering, the high surface energy contained in the powder leads to grain growth (reduction of surface area) by bonging of particles in direct contact through atomic diffusion. Grain growth and aggregation result in densification and strength augment of the green body.

Traditionally, dry- and hot-pressing followed by sintering have been the most commonly employed methods for the fabrication of polycrystalline CCO ceramics. In the last few years, though, newer and more sophisticated methods that combine pressing and sintering steps in one single operation have been increasingly used by scientists for fabrication of polycrystalline CCO. This is the case of high-pressure sintering and spark plasma sintering methods. Discussion of hot-pressing and spark plasma sintering methods will be extended in the following sections.

\subsubsection{Hot-pressing}

Hot-pressing is a densification method that allows controlling the microstructure of the ceramics by the application of uniaxial pressure assisted by heat. This method is employed when 
high density is required along with a textured microstructure (i.e., grain alignment). Additionally, hot-pressing produces homogeneous and low porosity products with superior mechanical properties. The hot-pressing is performed by two aligned rigid punches that compact the ceramic powder contained within a die while heat is provided to the compact by electrical resistance heating, induction heating or radiation. The applied stress produces rearrangement and plastic flow of the ceramic particles, while the heat promotes atomic diffusion [44]. A schematic view of the hot-pressing process is presented in Figure 5. The hot-pressing process can be adapted to simultaneously perform pressing and sintering when compositions with superior density and hardness are required. For that purpose, the pressing/heating hold time is extended sufficiently to allow grain coarsening to occur. Such compositions, however, may result too brittle requiring a post-sintering annealing treatment in order to relief internal stresses and reduce the brittleness.

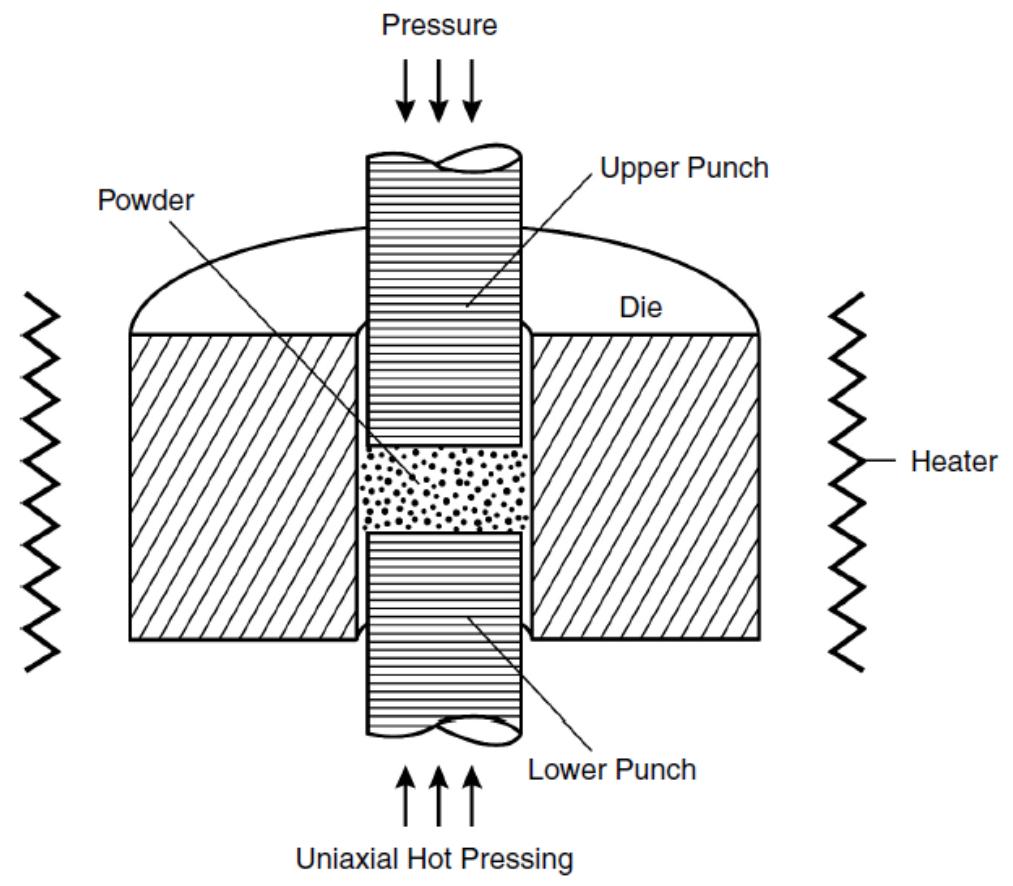

Figure 5. Schematic view of the hot-pressing process during powder compaction. Figure by Groza, J.R.[44]. 


\subsubsection{Spark plasma sintering}

Spark plasma sintering (SPS) is a consolidation method used to obtain highly dense bulk ceramic materials. This technique consists in the generation of a high-temperature spark plasma between the grains of the ceramic powder while it is pressed within a punch and die assembly. The spark plasma is generated by the application of a pulsed high-frequency electric current discharge that passes through the die. The plasma generated between the powder grains produce a rapid and severe heating that intensifies the sintering rate. Compared to the conventional densification/sintering methods such as hot-pressing, SPS can consolidate ceramics with higher density (larger than $99 \%$ of the theoretical density) in shorter times and at lower temperatures [45].

\subsection{Thermoelectric Properties Improvement}

The layered CCO ceramic system is well known to present a very remarkable TE performance for the single crystal. A $Z T$ value of 0.83 at $973 \mathrm{~K}$ has been reported by Shikano and Funahashi [6]. The single crystal ceramic, however, is not suitable for practical application in TE devices due primarily to production difficulties and high costs. For that reason, several strategies have been developed to improve the TE performance of polycrystalline $\mathrm{CCO}$, which is easier and cheaper to produce. The most significant approaches to optimize the TE performance of the polycrystalline ceramic are presented in the following sections.

\subsubsection{Fabrication method}

Grain alignment is known to be an effective way to reduce the electrical resistivity of CCO ceramics while keeping $S$ unaltered. Prevel et al. have analyzed the effect of using thermo- 
forging processes for the consolidation of $\mathrm{CCO}$ ceramics [35]. According to their results, grain growth is favored by the hot-pressing sintering process due to the improved contact between grains when stress is applied. Ceramic densities of $94.5 \%$ of the theoretical value were obtained by hot-pressing the powders under $16 \mathrm{MPa}$; this is a substantial improvement respect to the $63 \%$ value for the non-pressed ceramic. Additionally to grain growth and density, the electrical conductivity of the $\mathrm{CCO}$ was also found to be improved by stress application. A substantial resistivity reduction for the pressed ceramics was obtained at low temperatures. This resistivity reduction was attributed to the ceramic densification and to the alignment of the CCO $a b$-planes produced by the hot-pressing. This also explains the resistivity anisotropy found in the pressed ceramics; resistivity in the direction parallel to the stress axis was found to be 4 times the resistivity at the perpendicular direction. Zhou et al. also reported the reduction of the resistivity of CCO samples consolidated by the combination of the magnetic alignment technique with spark plasma sintering [46].

\subsubsection{Ion doping}

Several research works have reported that the thermoelectric performance of the CCO can be modified by the addition of dopants [7-20]. Partial substitution of the $\mathrm{Ca}^{2+}$ and $\mathrm{Co}^{3+}$ cations can result in the improvement of the Seebeck coefficient and reduction of the resistivity and thermal conductivity through the enhancement of the carrier concentration and the modification of the carrier mobility. Different materials have been reported to successfully improve the thermoelectric performance of the CCO. For example, Nong et al. have investigated the effect of doping trivalent late rare earths (Dy, $\mathrm{Er}, \mathrm{Ho}$ and $\mathrm{Lu}$ ) in the $\mathrm{Ca}^{2+}$ site for $\mathrm{CCO}$ samples prepared by solid state reaction followed by hot-pressing [12]. An improvement of the Seebeck coefficient 
and power factor was obtained for most of the tested systems as can be observed in Figure 6 . The resistivity, however, was observed to increase for all the systems due to the deterioration of the CCO microstructure by the action of the dopants. Nevertheless, the overall effect on ZT was positive; a $Z T$ of 0.36 at $1073 \mathrm{~K}$ was obtained using $\mathrm{Lu}$.
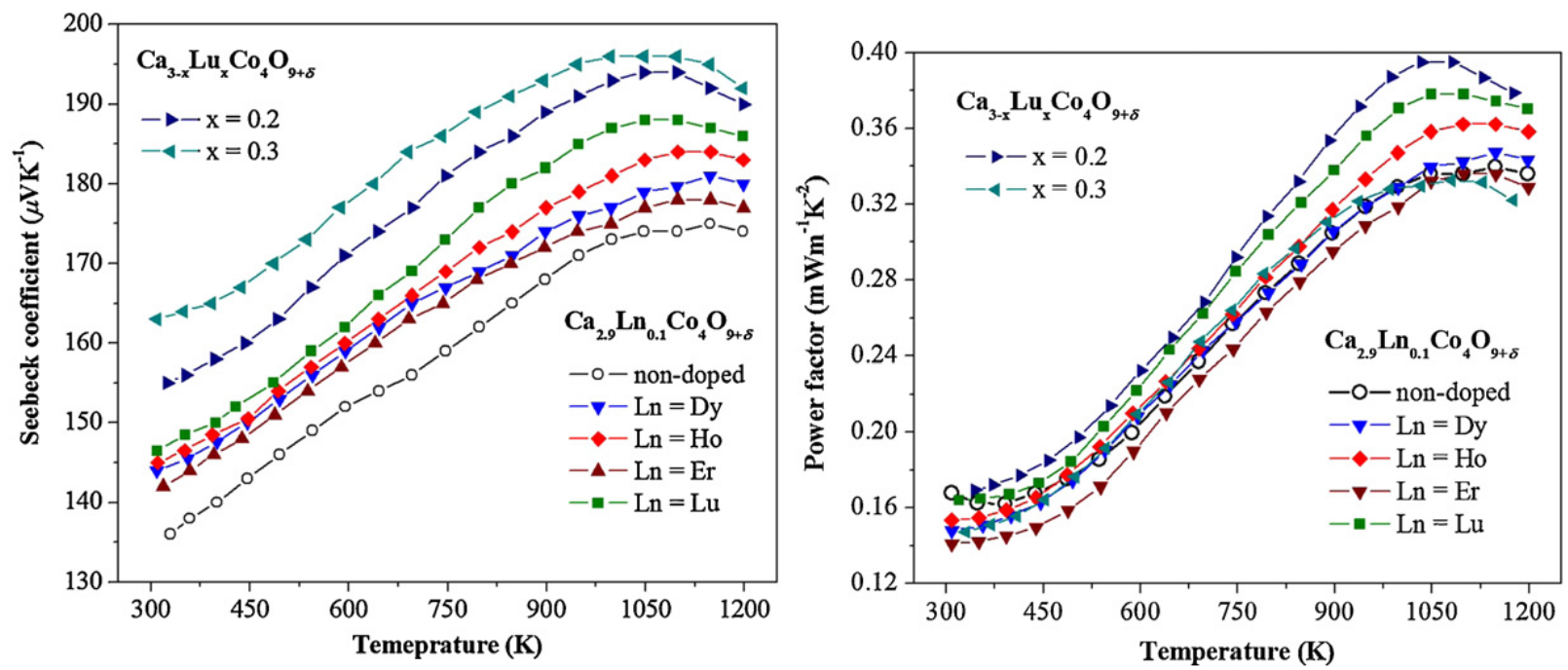

Figure 6. Improvement of the thermopower and power factor of $\mathrm{CCO}$ by doping late rare earths into the CCO. Source: Nong et al.[12].

Thermoelectric properties of Fe-doped $\mathrm{CCO}$ have been analyzed by Liu et al. at low temperature (from 0 to $300 \mathrm{~K}$ ) [11]. They prepared the $\mathrm{Ca}_{3} \mathrm{Co}_{4-\mathrm{x}} \mathrm{Fe}_{\mathrm{x}} \mathrm{O}_{9}$ system $(\mathrm{x}=0.05,0.1,0.15$ and 0.2 ) where $\mathrm{Fe}^{2+}$ partially substitutes $\mathrm{Co}^{3+}$. This substitution increased the conductivity of the $\mathrm{CCO}$ due to the increment of the hole carrier concentration. Although a reduction of the Seebeck coefficient was expected from the addition of hole carriers, an improvement of the thermopower was observed. Wang et al. also doped the CCO using Fe and also reported the simultaneous reduction of the resistivity and the increment of the Seebeck coefficient [17]. The reduction of the resistivity was attributed to the introduction of hole carriers by the $\mathrm{Fe}^{2+}$ substitution in the 
$\mathrm{Co}^{3+}$ site, while the improvement of the Seebeck coefficient was attributed to the enhancement of the electronic specific heat.

The use of Ag for doping the CCO has also been extensively studied. Wang et al. [18] reported a simultaneous improvement of the Seebeck coefficient, reduction of the resistivity and suppression of the thermal conductivity from the substitution of the $\mathrm{Ca}^{2+}$ by univalent $\mathrm{Ag}^{+}$. This substitution produces a variation of the Fermi level of the system (and consequently of the carrier mobility) which results in the improvement of the Seebeck coefficient. The reduction of the resistivity is attributed to the increment of the hole carriers resulting from the substitution.

Other transition metals like Yttrium have also been tested as CCO dopant. Wang et al. substituted $\mathrm{Y}^{3+}$ by $\mathrm{Ca}^{2+}$ to obtain the $\mathrm{Ca}_{3-\mathrm{x}} \mathrm{Y}_{\mathrm{x}} \mathrm{Co}_{4} \mathrm{O}_{9}$ system and measured its $\mathrm{TE}$ properties at high temperature [15]. This system offers a wide doping range due to the size match between the $\mathrm{Y}^{3+}$ and $\mathrm{Ca}^{2+}$ cations. The thermopower and the resistivity of the $\mathrm{CCO}$ were increased because of the reduction of the carrier concentration. Similar results were obtained by Liu et al. [19] for the $\mathrm{Ca}_{2.7} \mathrm{Y}_{0.3} \mathrm{Co}_{4} \mathrm{O}_{9}$ system; a reduction of the carrier concentration leading to the increment of the resistivity and thermopower.

\subsubsection{Incorporation of nanoinclusions}

The incorporation of nanoinclusions within the $\mathrm{CCO}$ is another strategy aimed to produce high performance polycrystalline CCO for the fabrication of practical TE devices [21-26]. The objective of this approach is to improve the electrical connections between the ceramic grains (resulting in the improvement of the electrical conductivity) and to reduce the thermal conductivity by phonon scattering effects. This technique is usually combined with doping of $\mathrm{CCO}$ to influence its electrical and thermal conductivities by acting on the carrier concentration 
and lattice parameters of the material. One example of this strategy was reported by Wang et al. where a $Z T$ of 0.5 was obtained at $1000 \mathrm{~K}$ for cold pressed polycrystalline $\mathrm{CCO}$ as the result of the combined doping and alloying action of $\mathrm{Ag}^{+}$in the $\mathrm{Ca}_{2.7} \mathrm{Ag}_{0.3} \mathrm{Co}_{4} \mathrm{O}_{9} / \mathrm{Ag}-10 \mathrm{wt} \%$ composite system [18].

Significant enhancements in the power factor of CCO ceramic by addition of metallic particles have been reported. Mikami et al. tested a hot-forged CCO/Ag composite containing dispersed Ag particles [21]. Addition of Ag resulted in a significant decrease of the electrical resistivity accompanied by a minor degradation of the Seebeck coefficient. The overall effect in the power factor, however, was favorable due to the dominant effect over $\rho$. The objective of this approach consists in reducing $\rho$ by the addition of a material with a lower $\rho$ at the grain boundaries of the ceramic in order to assist the electrical conductivity between grains. The optimal Ag amount was found to be $10 \mathrm{wt} \% \mathrm{Ag}$.

As stated by Kim et al. a substantial reduction of the thermal conductivity of semiconductors (by phonon scattering effects) can be achieved using substitutional alloying along with the incorporation of secondary-phase nanoinclusions [26]. The model suggests that at the "alloy limit", point defects produced by atomic substitutions can scatter short-wavelength phonons due to differences in mass and/or bond stiffness. In addition, mid-to-long-wavelength phonons are estimated to be scattered by the nanoparticles randomly incorporated throughout the material. The authors reported a significant reduction of $\kappa$ for the thermoelectric $\operatorname{In}_{0.53} \mathrm{Ga}_{0.47}$ As alloy system with epitaxially added ErAs nanoparticles. The combined action of both atomic substitution and nanoparticles resulted in a $Z T$ improvement by a factor of two.

Faleev and Leonard have presented a theoretical model (based on the band bending concept) for the enhancement of the TE properties of semiconductor materials containing randomly 
distributed metallic nanoinclusions [25]. This model deals with the electron scattering phenomenon occurring at the metal/semiconductor interfaces of metallic nanoinclusions within a bulk semiconductor material. According to the model, charge transfer at the metal/semiconductor interface produce a band bending away from it. This band bending is characterized by an electrostatic potential able to scatter low energy electrons resulting in an energy filtering effect that favors the Seebeck coefficient. Additionally, a reduction of thermal conductivity by scattering of mid-to-long-wavelength phonons is also attributed to nanoinclusions in crystalline semiconductors [26]. The combined action of $S$ improvement along with the $\kappa$ reduction caused by electron scattering and phonon scattering respectively, can result in a substantial $Z T$ improvement by much as $430 \%$ for semiconductor/metallic-nanoinclusions systems as claimed by Faleev and Leonard. One significant benefit from this model is that allows determining the optimal parameters of the nanoinclusions for the design of superior thermoelectric semiconductors, such as volume fraction or radius. Finally, the authors employed nanoinclusion radii sizes between 2.5 and $6 \mathrm{~nm}$, along with a volume fraction of $5 \%$ for the reported calculations. It is important to highlight, though, the difficulty that represents to obtain such small nanoparticles uniformly distributed into a bulk material.

Finally, Nong et al. have reported the formation of metallic nanoinclusions within the $\mathrm{Ca}_{2} \mathrm{CoO}_{3}$ subsystem from $\mathrm{Ag}^{+}$and $\mathrm{Lu}^{3+}$ doping, for SPS processed specimens [22]. From the XRD and TEM analyses of a synthesized $\mathrm{Ca}_{2.8} \mathrm{Ag}_{0.05} \mathrm{Lu}_{0.15} \mathrm{Co}_{4} \mathrm{O}_{9+\delta}$ system could be concluded that $\mathrm{Ag}^{+}$precipitated as a secondary phase (that agglomerated at the grain boundaries) after lattice saturation. $\mathrm{Lu}^{3+}$ on the other hand, fully substituted the $\mathrm{Ca}^{2+}$ site since no evidence of an additional secondary phase was found. A $Z T$ value of 0.61 at $1118 \mathrm{~K}$ was obtained for this system which is very superior to the $Z T$ value of 0.25 of the non-doped specimen. TE analysis of 
the doped system showed an improvement of $S$ along with a reduction of $\rho$ and $\kappa$. The improvement of $S$ as well as the suppression of $\kappa$ was attributed to the effect of $\mathrm{Ca}^{2+}$ substitution by $\mathrm{Ag}^{+}$and $\mathrm{Lu}^{3+}$ while the $\rho$ reduction was attributed to the electric connector action of the $\mathrm{Ag}$ phase precipitated between the cobaltite grains. 


\section{CHAPTER 3: EFFECT OF THE SOL-GEL SOLVENT ON THE THERMOELECTRIC PROPERTIES OF PURE $\mathrm{Ca}_{3} \mathrm{Co}_{4} \mathrm{O}_{9}$}

CERAMICS

\subsection{Experimental Procedure}

In order to compare their $\mathrm{TE}$ properties, three different experimental $\mathrm{CCO}$ ceramics were produced applying the sol-gel route followed by hot pressing and sintering processes. The main difference between their fabrication routes was the solvent used as gel precursor: the first CCO was prepared using deionized water as solvent, the second one used a mixture of deionized water and ethylene glycol as solvent (50\%-50\% in volume) and the third one was prepared using diethylene glycol as solvent. The samples will be denoted as: DI water CCO, EG-50\% CCO and DEG CCO respectively.

In order to produce the three $\mathrm{CCO}$ powders, aqueous solutions were prepared by dissolution of citric acid monohydrate, ethylene glycol, polyethylene glycol and nitric acid into the respective solvents. The addition of metal precursor salts (calcium nitrate tetrahydrate and cobalt nitrate hexahydrate) in stoichiometric ratios ( $\mathrm{Ca}$ to $\mathrm{Co}$ ratio of 3:4) completed the solutions. Small amounts of polyethylene glycol (PEG) and ethylene glycol (EG) were added for all the solutions as dispersants while nitric acid was used as peptizer agent. Additionally, citric acid monohydrate was used as complexing agent.

The resulting solutions were thoroughly mixed and stirred by three hours at $80^{\circ} \mathrm{C}$ and 200 rpm, until gel viscosity reached its maximum. Then the viscous solutions were dried in a box furnace at $500^{\circ} \mathrm{C}$ by two hours in order to volatize all the organic matter contained in the 
mixture. The resulting ashes were subsequently grounded before performing calcination in a tube furnace with oxygen at $650^{\circ} \mathrm{C}$ during four hours. The calcined powders were uniaxially hotpressed into pellets under $1 \mathrm{GPa}$ pressure at $150^{\circ} \mathrm{C}$ during 30 minutes. Finally, the pressed pellets were sintered in a box furnace during 18 hours at $920^{\circ} \mathrm{C}$. A flow diagram of the previously described procedures is presented in Figure 7.

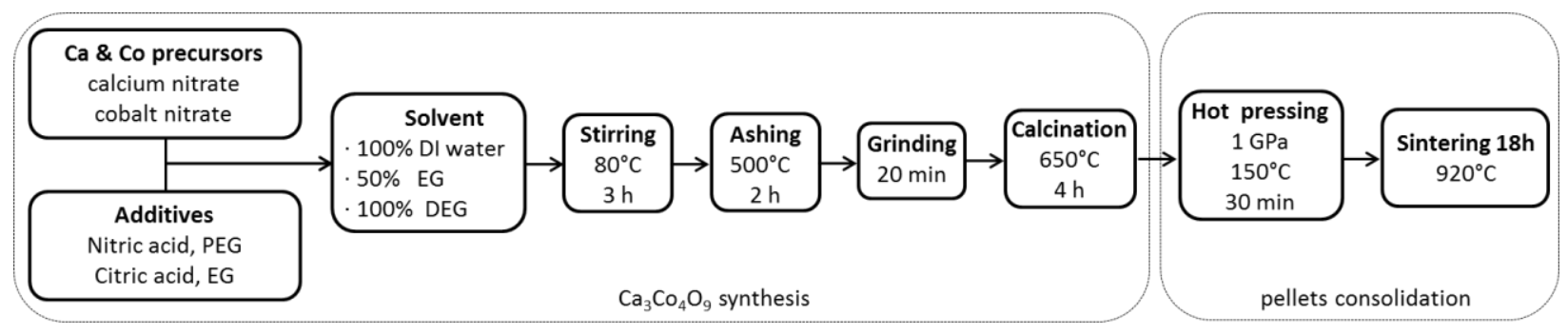

Figure 7. Flow diagram of the fabrication route of the three pure CCO ceramics synthesized: DI water CCO, EG-50\% CCO and DEG CCO. The fabrication routes differ in the solvent employed.

In order to observe the morphology and texture of the synthesized ceramic powders and pellets, a SEM study of the samples was performed using a JEOL JSM-7600F electron microscope. Two different surfaces of the pellets were imaged: the pressed surface and a fractured cross-section. Additionally, TEM examination of the ceramic pellets was also performed to detail the composition and crystal structure of the materials at the nano-scale. TEM analysis was performed using a JEOL TEM-2100 equipment operating at $200 \mathrm{kV}$ (with point resolution of $0.23 \mathrm{~nm}$ ) equipped with energy dispersive spectroscopy (EDS) analyzer. The TEM specimens were prepared by conventional polishing of the samples using abrasive films. Indexing of the electron diffraction patterns was assisted by the ICDD PDF-4+ version 4.1203 software. To conclude, $S$ and $\rho$ of the ceramics were measured in the direction parallel to the 
pressed plane from room temperature to $800^{\circ} \mathrm{C}$ using a Linseis LSR-1100 Seebeck \& Electric Resistivity Unit in a He environment.

\subsection{Results and Discussion}

Synthesized ceramic powders were imaged using SEM before pellet consolidation. The three ceramic powders present irregular shapes and wide size distribution as can be observed in Figure 8. No major differences arising from the use of different solvents are noticeable from the SEM micrographs.
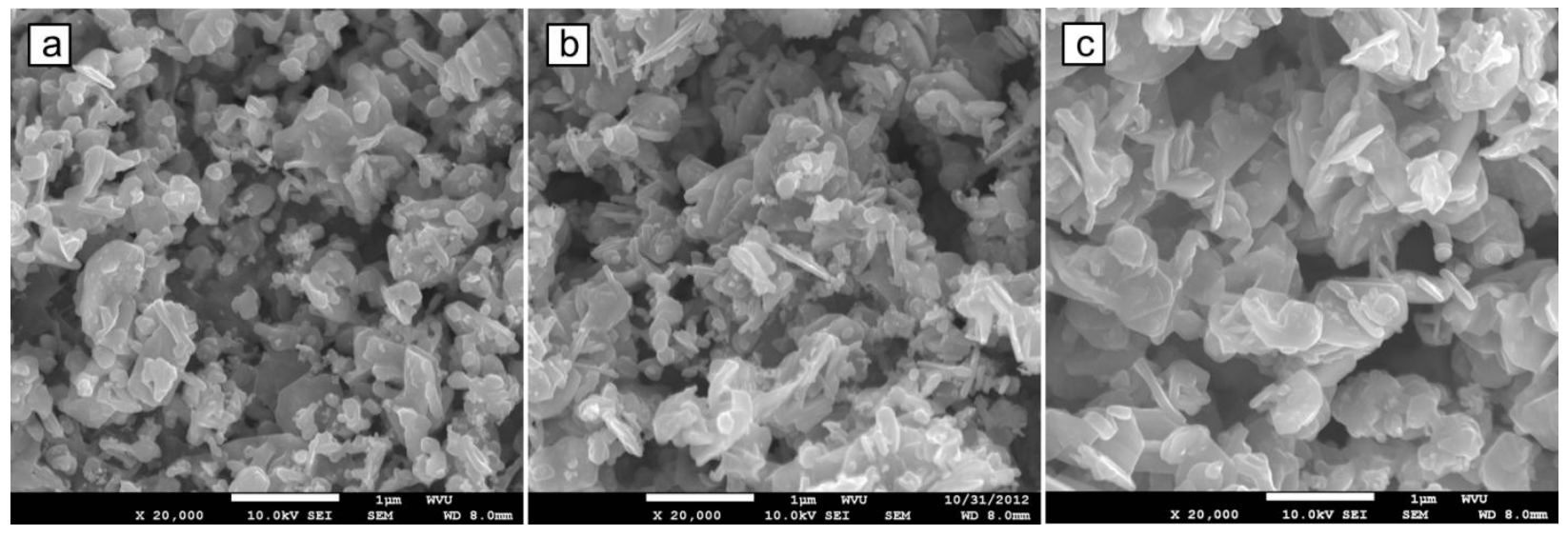

Figure 8. SEM micrographs of the synthesizes pure CCO powder using different solvents (a) DI water (b) EG-50\% and (c) DEG.

SEM micrographs from the pressed plane and fractured cross-sectional surface of the DI water CCO, EG-50\% CCO and DEG CCO samples are presented in Figure 9. The size and shape of the ceramic grains as well as the ceramic texture after hot-pressing and sintering can be appreciated. In terms of morphology, the three ceramics present plate-like grains; with rounded shapes when observed from top (pressed plane) and elongated shapes in the lateral view (crosssection). The ceramic texture can also be appreciated with the platelets tending to be aligned perpendicular to the applied stress axis. 
In general, the powder morphology as well as the ceramic microstructure and texture of the three ceramic samples are very similar; no significant differences are evident in the SEM micrographs. The alignment of all the samples is poor and porosity is high, characteristics that negatively affect the thermoelectric properties of the ceramic. It is believed that the alignment and high density produced by hot-pressing the powders got deteriorated during the sintering process where residual stresses were released and grain growth occurred without mechanical constraints. According to this, a simultaneous pressing-sintering process is expected to produce ceramics with superior texture and density.

TEM analysis of the samples was also performed in order to reveal the nanostructure of the ceramics. TEM micrographs allow, in more detail, to observe the polycrystalline structure of the material, the grain alignment and the porosity. Figure 10 shows some representative features of the nanostructure of the DI water CCO. Figure 10 (a) shows a typical CCO grain composed by numerous, well defined CCO layers or nano-lamellas stacked up as can be observed detailed in Figure 10 (b). The nano-lamellas are the well-defined, different-contrast strips constituting the crystal grain. Furthermore, the regular atomic planes building up the nano-lamellas can be observed as the parallel lines within the nano-lamellas. The main characteristic of these grains is that all the nano-lamellas have the $c$-axis parallel and, at the same time, perpendicular to their longer dimension [9]. The different contrast between the nano-lamellas arises from different orientations of the $a b$-planes. It can also be observed that nano-lamellas vary in thickness from very thin ones made up by just a couple of crystal planes, to much thicker ones comprising hundreds of crystal planes. Figure 10 (c) presents an electron diffraction pattern taken from the imaged CCO grain; it corresponds to the $[1 \overline{1} 0]$ zone axis of the monoclinic $\mathrm{Ca}_{3} \mathrm{Co}_{4} \mathrm{O}_{9}$ system. The direction of the $c$-axis is indicated in it. 

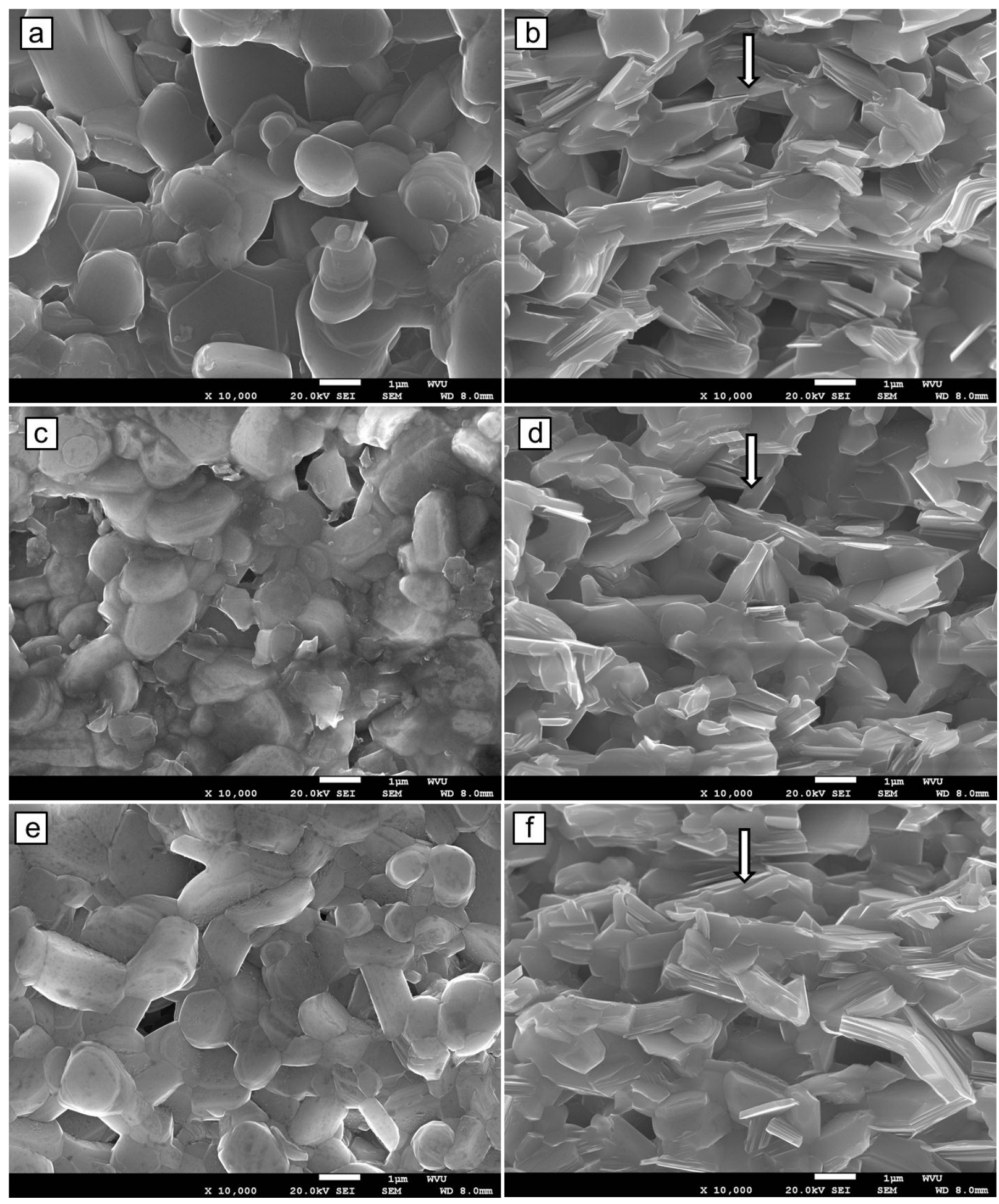

Figure 9. SEM micrographs from the pressed plane and cross-sectional fractured surface of the

DI water CCO (a) and (b), EG-50\% CCO (c) and (d), and DEG CCO (e) and (f). The arrows indicate the direction of the applied pressure. 

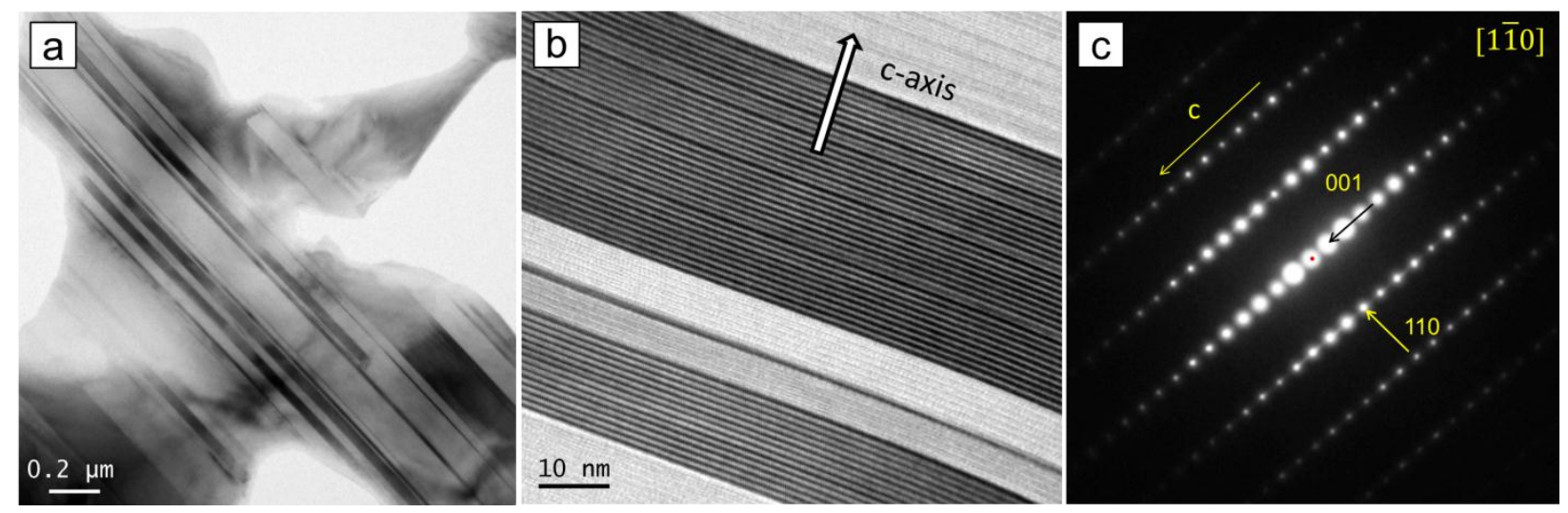

Figure 10. Nanostructure of the DI water CCO: (a) CCO grain composed by nano-lamellas, (b)

nano-lamellas made up by crystalline planes and (c) electron diffraction pattern from the grain.

The EG-50\% and DEG CCO samples were also imaged using TEM. Figure 11 and Figure 12 show that these two ceramics are also composed by layered $\mathrm{Ca}_{3} \mathrm{Co}_{4} \mathrm{O}_{9}$ grains. Nano-lamellas of these ceramics are comparable to those observed in the DI water CCO ceramic.
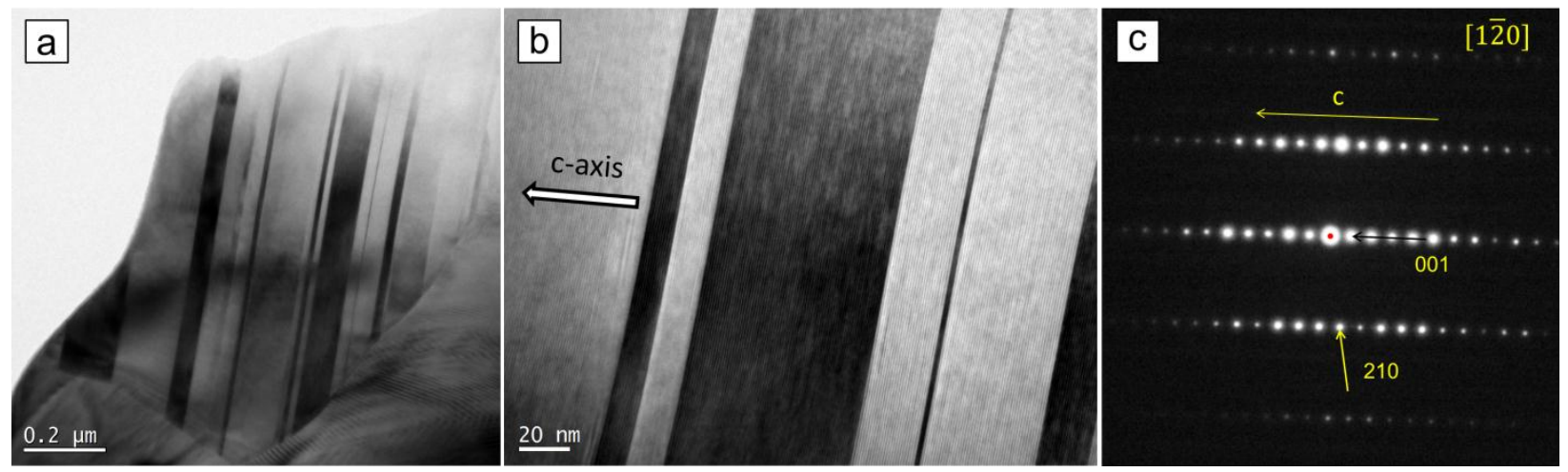

Figure 11. Nanostructure of the EG-50\% CCO: (a) CCO grain composed by nano-lamellas, (b) bundle of nano-lamellas and (c) its corresponding electron diffraction pattern. 

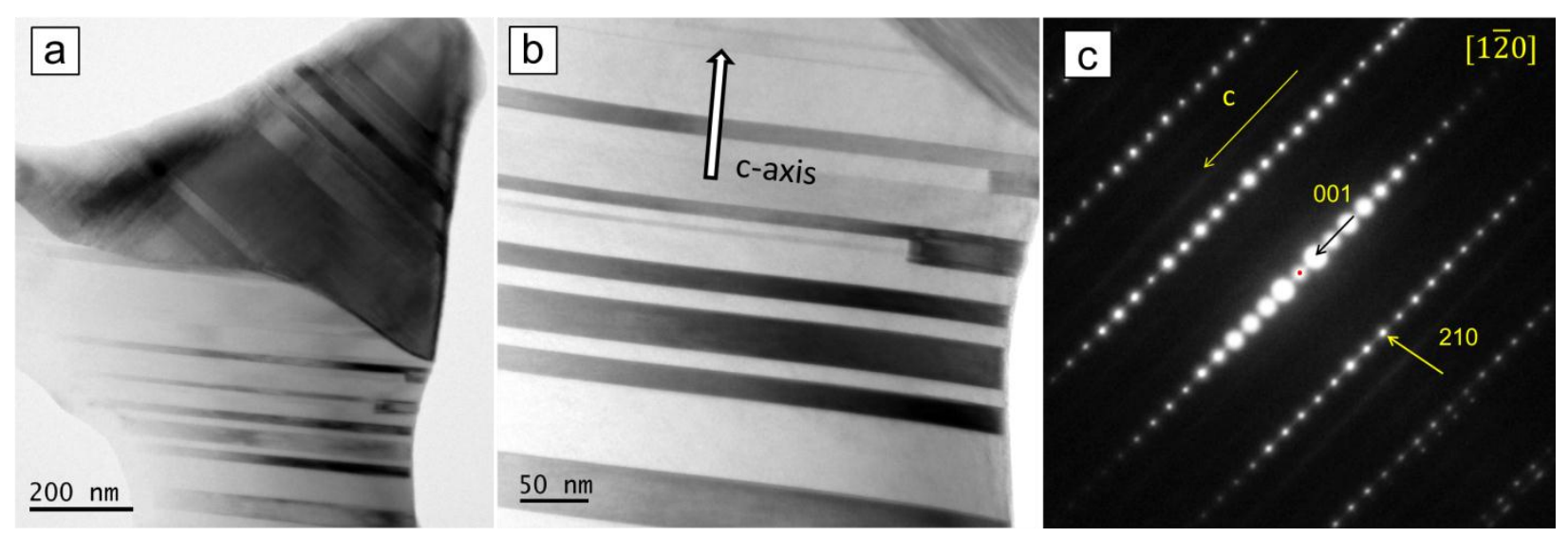

Figure 12. Nanostructure of the DEG CCO: (a) CCO grains composed by nano-lamellas, (b) bundle of nano-lamellas and (c) and electron diffraction pattern.

TEM-EDS analysis revealed the existence of crystalline cobalt oxide grains in the three experimental CCO samples as can be observed in Figure 13. Image (a) shows a cobalt oxide grain found within the $\mathrm{CCO}$ in the DI water CCO sample, (b) presents an electron diffraction pattern taken from this crystal and was indexed as the [001] zone axis of the cubic CoO system. The grain enclosed in (c) was imaged in the EG-50\% CCO sample; its electron diffraction pattern (d) was indexed as the [111] zone axis of the cubic $\mathrm{Ca}_{3} \mathrm{O}_{4}$ system. (e) and (f) correspond to the DEG CCO sample, the imaged crystallite was also characterized as cubic $\mathrm{Ca}_{3} \mathrm{O}_{4}$. These crystalline grains were observed all through the ceramic samples; their occurrence, however, is known to be common in the CCO ceramics [14]. 

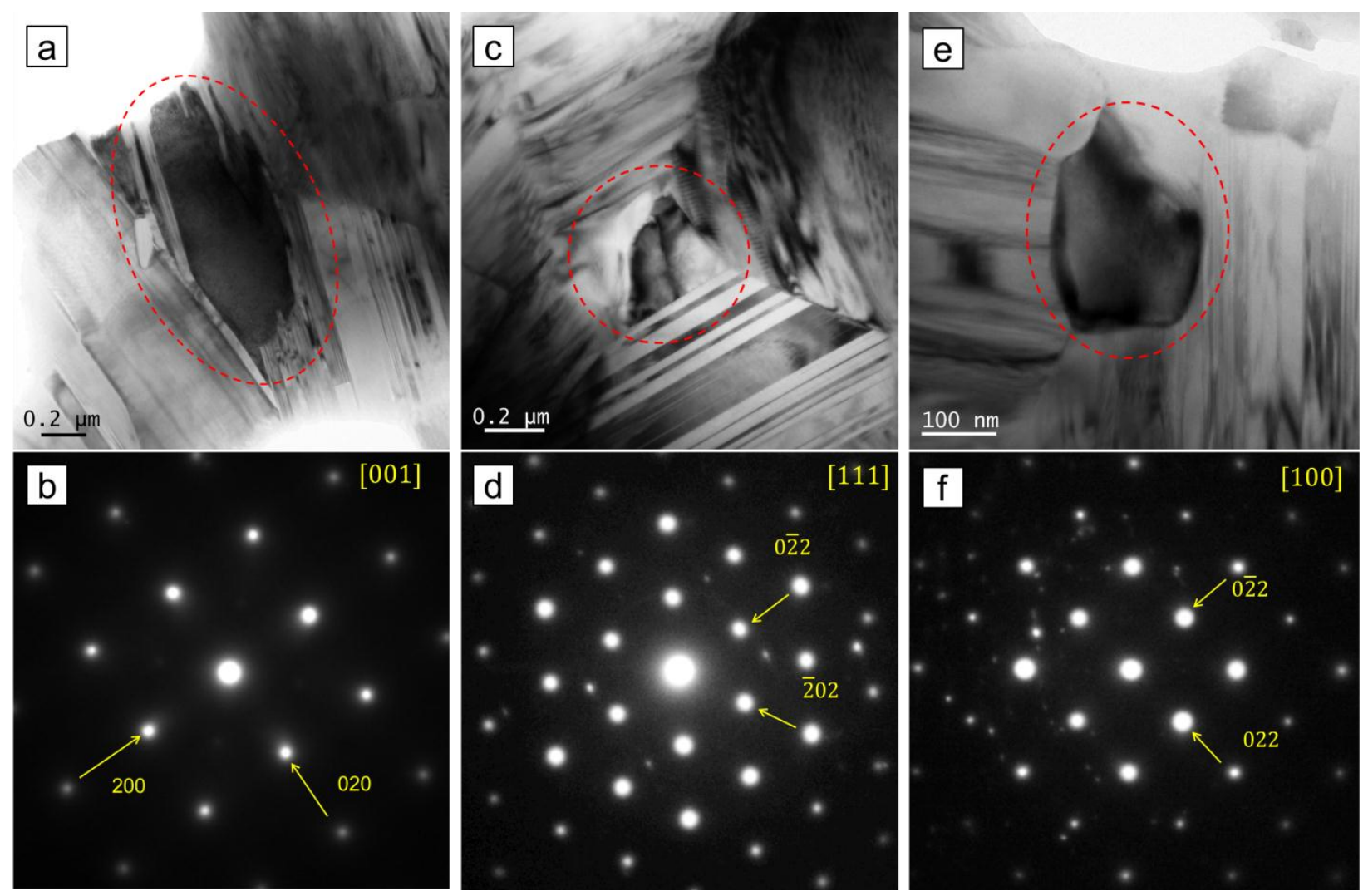

Figure 13. TEM micrographs of cobalt oxide grains with their respective electron diffraction patterns from: (a) and (b) DI water CCO sample, (c) and (d) EG-50\% CCO sample, and (e) and (f) DEG CCO sample.

$S$ and $\rho$ of the CCO samples were measured from room temperature to $800^{\circ} \mathrm{C}$ and power factors were calculated. The corresponding $S-T, \rho-T$ and power factor- $T$ curves are presented in Figure 14. For the three samples, the thermopower curves increase with temperature and show ptype thermoelectric behavior (positive $S$ for the entire temperature interval measured) indicating that hole carriers dominate the transport properties of the materials [7]. The best thermopower performance was obtained from the EG-50\% CCO sample which presents the largest $S$ above $330^{\circ} \mathrm{C}$. At the highest temperature measured $\left(800^{\circ} \mathrm{C}\right)$ the largest $S$ corresponds to this sample with $200 \mu \mathrm{VK}^{-1}$ while the DEG CCO sample presents the lowest thermopower value of 168 
$\mu \mathrm{VK}^{-1}$. The DI water CCO sample presents $S$ values just above of those of the DEG CCO sample at $800^{\circ} \mathrm{C}$. Below $330^{\circ} \mathrm{C}$, however, the DEG CCO sample presents the best performance.

Resistivity curves of the different samples present different transport behaviors, with marked nonmetal to metal transitions at elevated temperatures. Below $450^{\circ} \mathrm{C}$, the $\mathrm{EG}-50 \% \mathrm{CCO}$ presents the lowest resistivity values while the DEG CCO does above this temperature. Resistivity values for the DI water $\mathrm{CCO}$ are the largest up to $600^{\circ} \mathrm{C}$; over this temperature the $\mathrm{EG}-50 \% \mathrm{CCO}$ sample present the largest values. For the measured temperature range, the EG-50\% CCO shows the largest power factor followed by the DEG CCO sample (up to $730^{\circ} \mathrm{C}$ ). The DI water CCO, consequently, presents the lowest power factor.

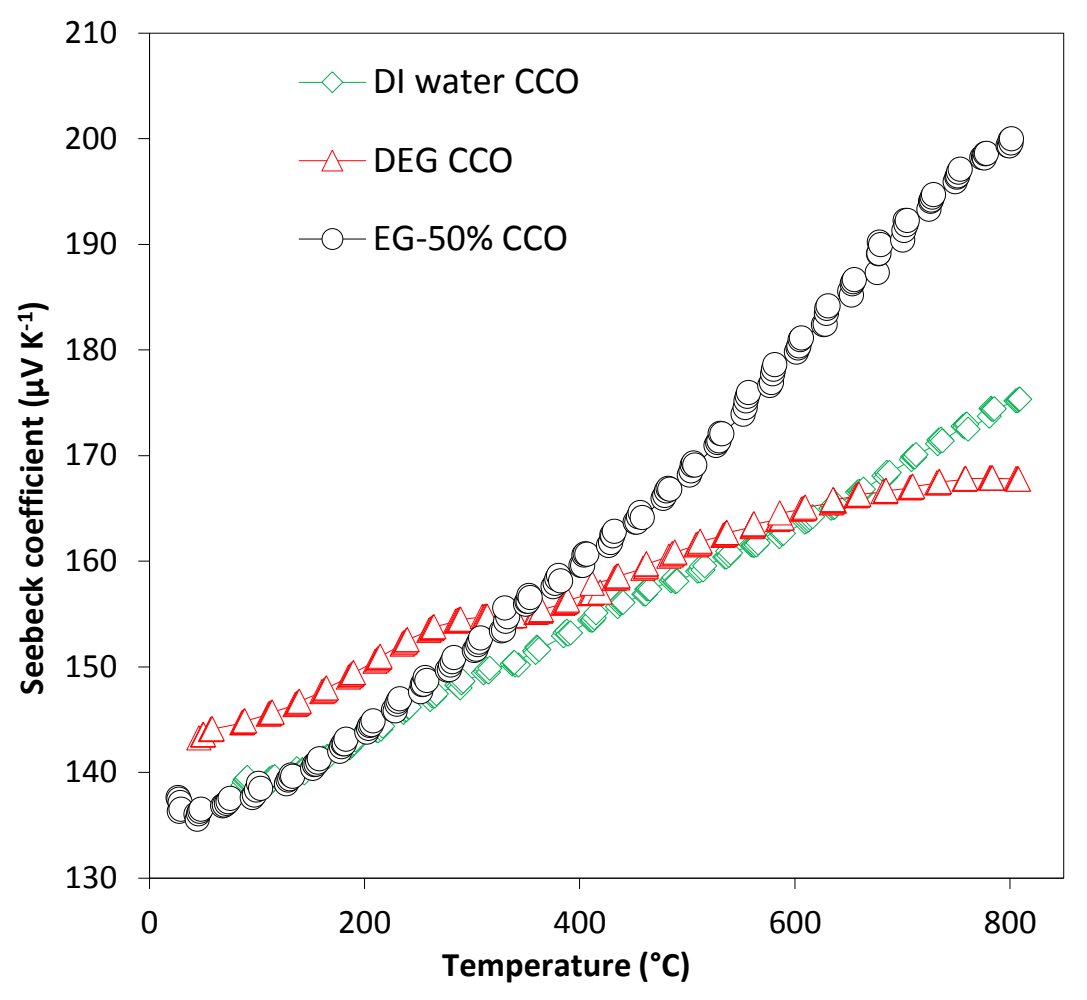



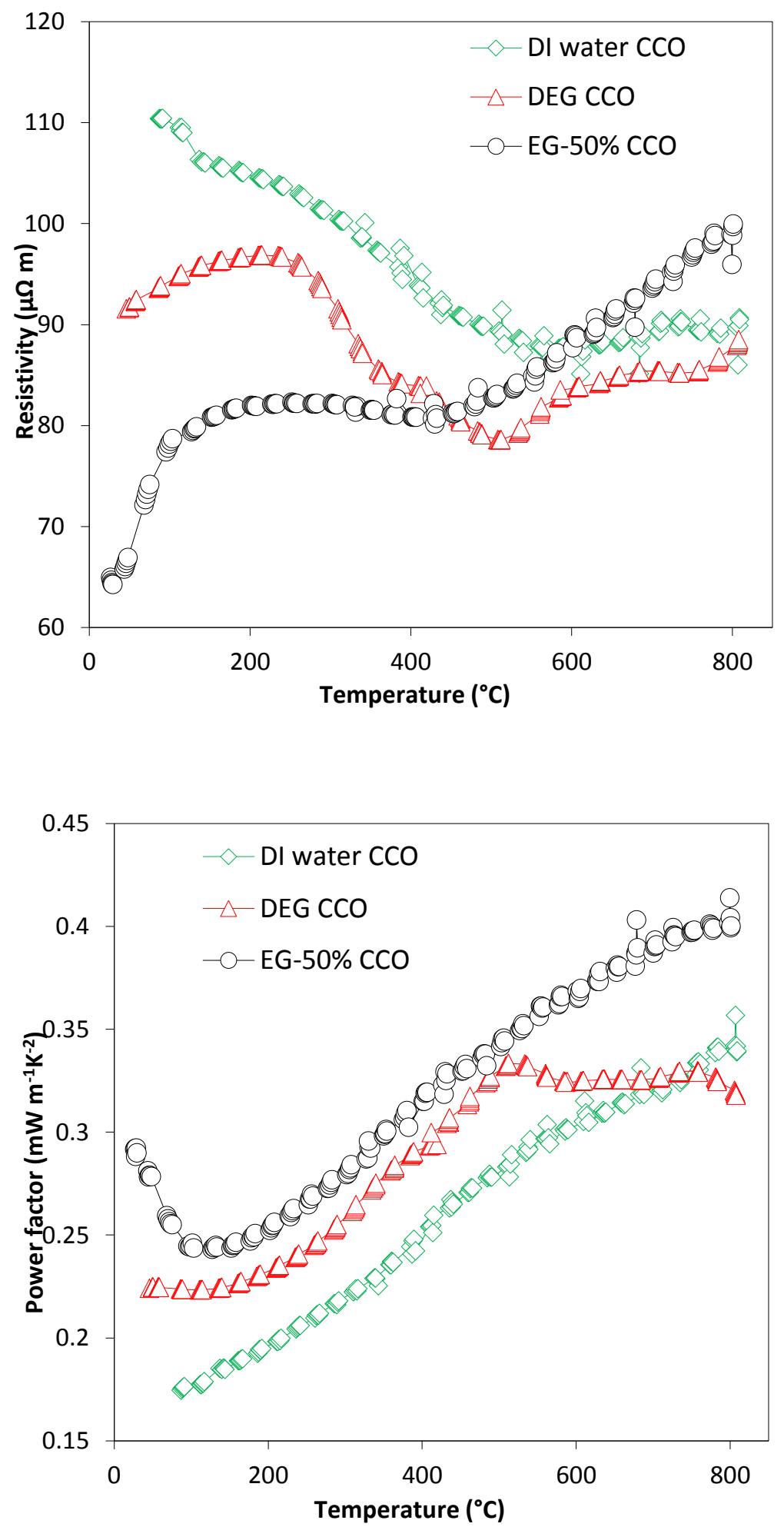

Figure 14. Seebeck coefficient, resistivity and power factor curves of the three experimental CCO samples. 


\section{CHAPTER 4: EFFECT OF THE ADDITION OF OXIDE}

\section{CERAMICS ON THE THERMOELECTRIC PROPERTIES OF}

\section{$\mathrm{Ca}_{3} \mathrm{Co}_{4} \mathrm{O}_{9}$ CERAMICS}

\subsection{Effect of YSZ Addition on the Thermoelectric Properties of $\mathrm{Ca}_{3} \mathrm{Co}_{4} \mathrm{O}_{9}$ Ceramics}

\subsubsection{Experimental procedure}

The experimental composite ceramic system $\mathrm{Ca}_{3} \mathrm{Co}_{4} \mathrm{O}_{9} / \mathrm{YSZ}-2$ wt $\%$ was synthesized by the sol-gel route with the addition of Yttria-Stabilized Zirconia $\left[\left(\mathrm{Y}_{2} \mathrm{O}_{3}\right)_{0.08}\left(\mathrm{ZrO}_{2}\right)_{0.92}\right](8$ mole \%) nanopowders during the sol-gel solution preparation. Precursor sol-gel solution was prepared using the same procedure employed for the synthesis of the pure CCO system using deionized water as solvent, plus the addition of the respective amount of YSZ nanopowders in order to obtain the desired weight ratio of $2 \mathrm{wt} \%$ YSZ. The resultant sol-gel solution was processed until pellet consolidation. The flow diagram of the entire process is illustrated in Figure 15. An additional CCO ceramic without YSZ was prepared under the same conditions to serve as baseline for the $S, \rho$ and power factor measurements.

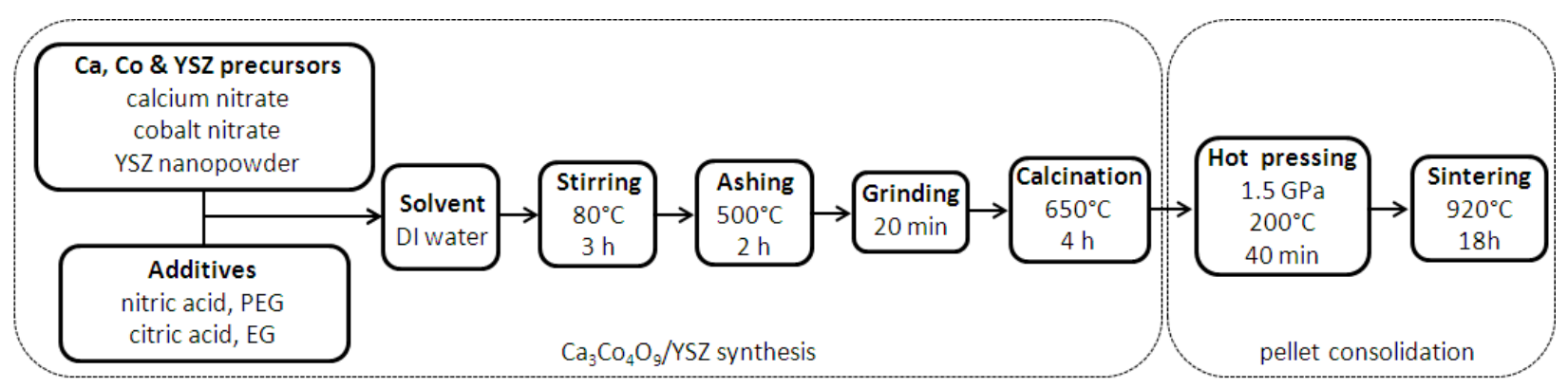

Figure 15. Flow diagram of the fabrication route of the CCO/YSZ-2 wt\% ceramic composite. 
The synthesized powder as well as the pressed plane and fractured cross-section of the alloyed ceramic were imaged using a JEOL JSM-7600F SEM. TEM analysis of the composite ceramic was also executed using a JEOL TEM-2100 coupled with EDS analyzer. The TEM specimens were prepared by conventional polishing of the samples using abrasive films. Indexing of the electron diffraction patterns was assisted by the ICDD PDF-4+ version 4.1203 software. Lastly, a Linseis LSR-1100 unit was employed to simultaneously measure $S, \rho$ and power factor of both the pure $\mathrm{CCO}$ ceramic and the CCO/YSZ-2 wt\% composite. Measurements were performed in the direction parallel to the pressed plane from room temperature up to $800^{\circ} \mathrm{C}$ in a $\mathrm{He}$ environment.

\subsubsection{Results and discussion}

The calcined CCO/YSZ-2 wt\% ceramic powder was imaged using SEM before pellet consolidation. A fine and homogeneous powder was obtained. The powder is composed by faceted plate-like crystal grains with size below $1 \mu \mathrm{m}$. A micrograph of a powder cluster can be appreciated in Figure 16.

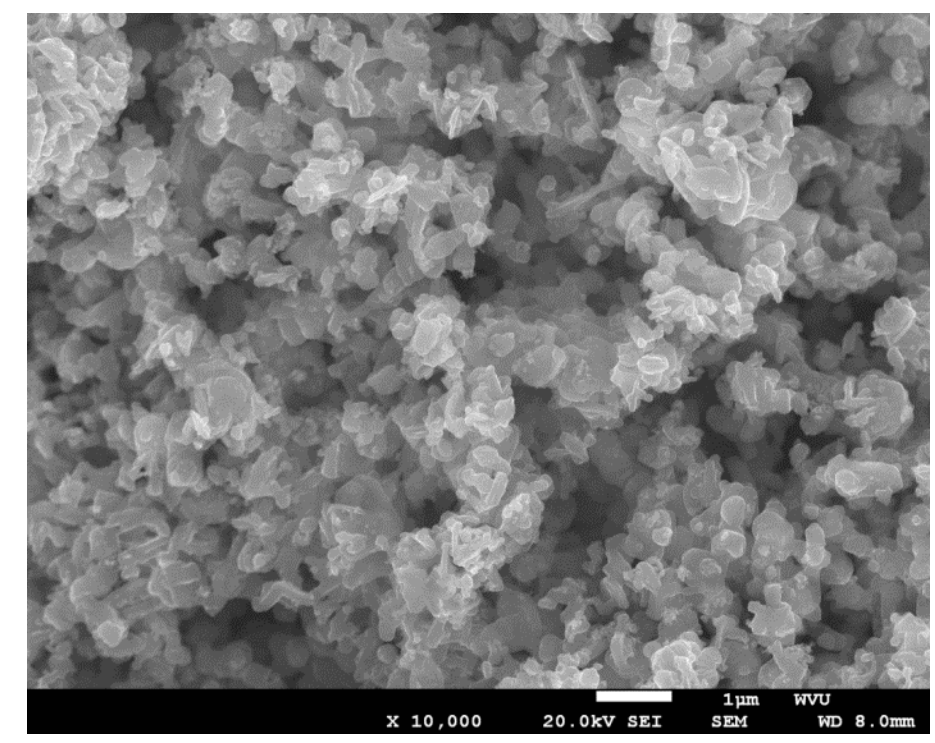

Figure 16. SEM micrograph of the synthesized CCO/YSZ ceramic powder before consolidation. 
As mentioned before, the composite ceramic was also imaged using SEM; Figure 17 shows the microstructure of two different surfaces: the pressed plane (a) and the fractured cross-section (b). The difference between the two surfaces denotes the texture produced by the stress exerted to the powders during pellet consolidation. The pressed plane image (a) presents the characteristic rounded shape of the pressed ceramic grains when observed from top. No prefferential alignment of the material is noticeable. Cross-sectional image (b), in contrast, shows the elongated plate-shaped ceramic grains aligned perpendicular to the applied stress direction. The ceramic grains present thicknesses under $1 \mu \mathrm{m}$ and lengths of several microns.
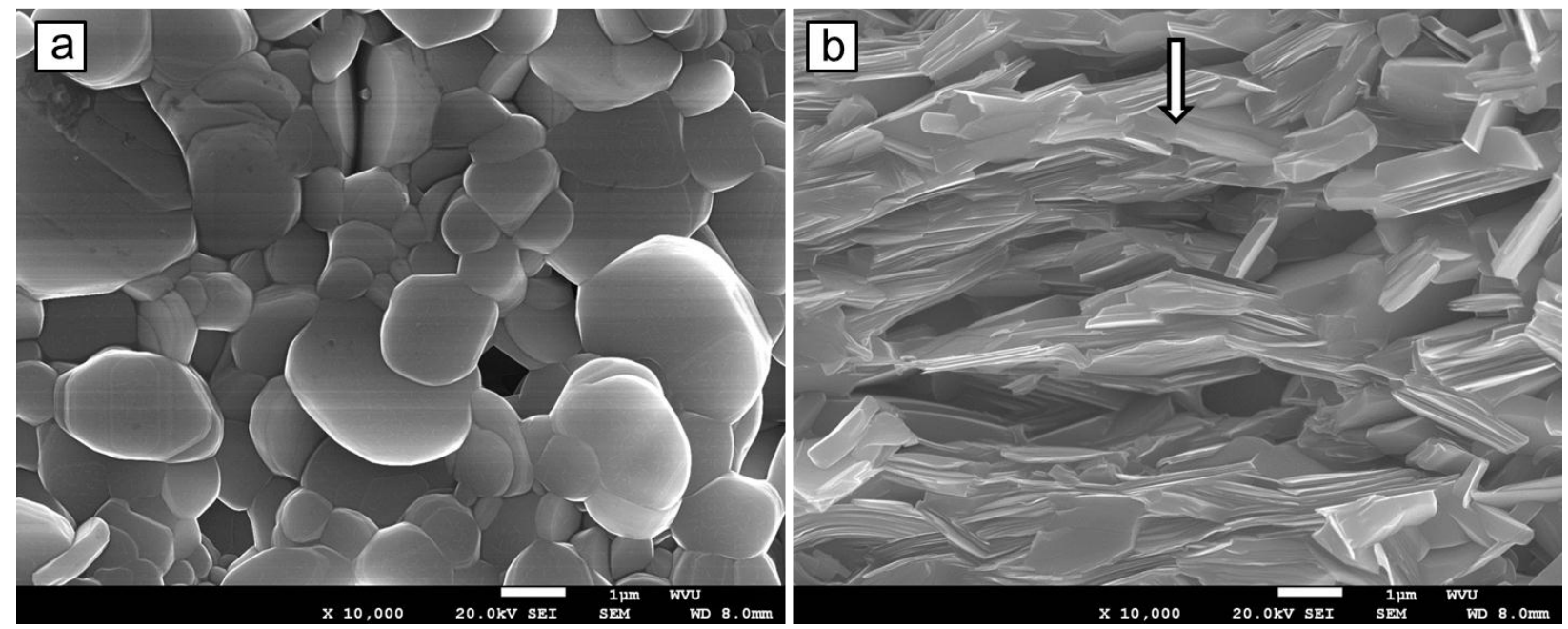

Figure 17. SEM micrographs of the morphology of the CCO/YSZ composite showing: (a) the pressed plane and (b) the fractured cross-section. The arrow indicates the applied stress direction.

TEM analysis of the specimen revealed the nanostructure of the CCO/YSZ composite ceramic. Figure 18 (a) shows a polycrystalline region with grains composed by bundles of nanolamellas and (b) shows the atomic planes that constitute the nano-lamellas. The EDS chemical analysis revealed the presence of $\mathrm{Zr}$ in the elemental composition of the crystals (see Table 1). This fact indicates that $\mathrm{Zr}$ doped the $\mathrm{CCO}$ material. 


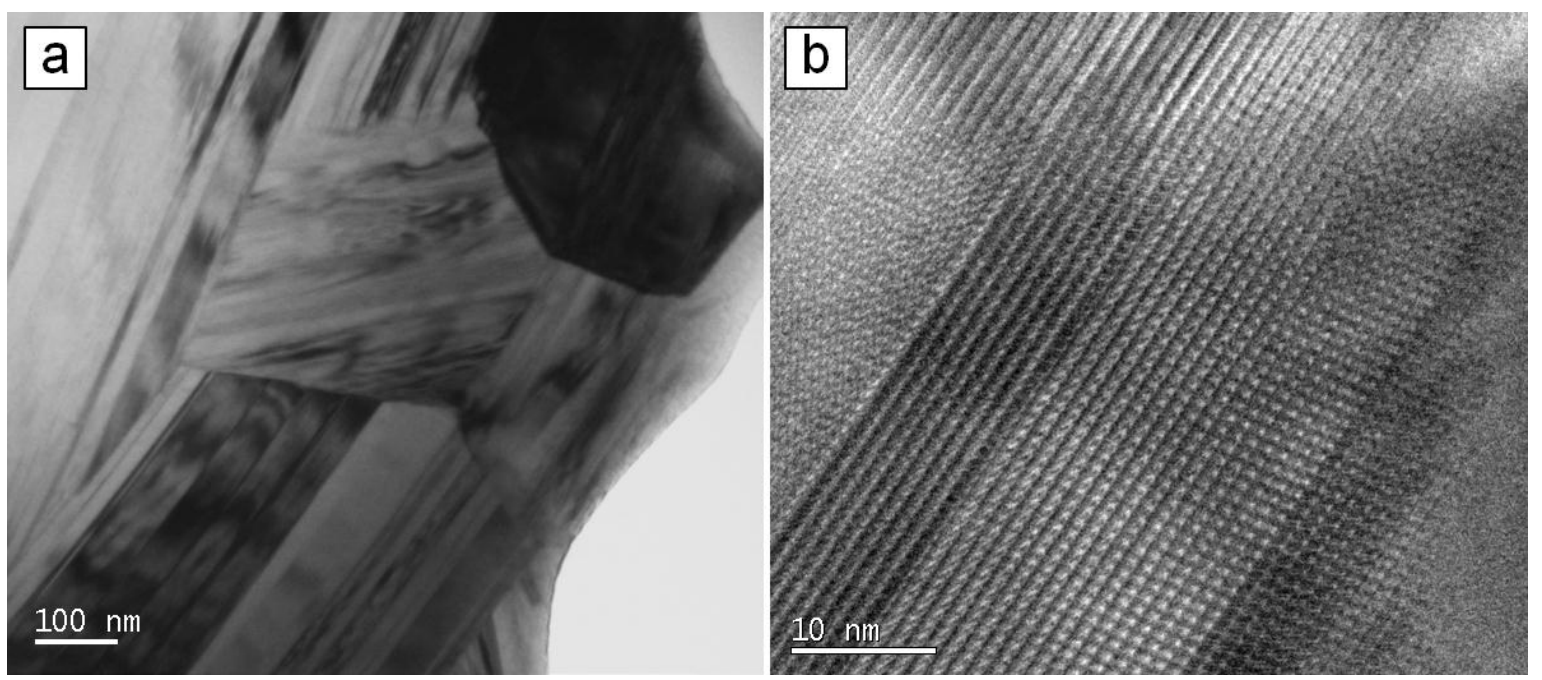

Figure 18. TEM micrographs from Ca3Co4O9/YSZ ceramic: (a) polycrystalline region and (b)

HRTEM image of the atomic planes.

Table 1. Representative EDS data from the polycrystalline region of the CCO/YSZ ceramic.

\begin{tabular}{|c|c|c|c|}
\hline \multicolumn{4}{|c|}{ atomic \% } \\
\hline O & Ca & Co & $\mathrm{Zr}$ \\
\hline 46.83 & 21.97 & 30.94 & 0.25 \\
\hline
\end{tabular}

The CCO/YSZ composite nanostructure, however, is not entirely uniform as shown in the previous image. Further TEM examination revealed the existence of YSZ clusters and regions (with several microns in length) of interpenetrated CCO and YSZ crystals. It can be observed in Figure 19Figure 19 where CCO grains appear clearly affected by the penetrating action of YSZ. Nonstoichiometric CCO grains with $\mathrm{Ca}$ or $\mathrm{Co}$ deficiencies coexist in these regions as evidenced by the EDS analysis presented in Table 2 where the Ca to Co ratio for the CCO widely vary from grain to grain. As a consequence, the neat configuration of the ceramic planes into nano-lamellas was severely impaired by the YSZ action. The nanostructure of these clusters is composed by 
polycrystalline nonstoichiometric CCO-YSZ regions with highly defective lattice arrangements as detailed in Figure 19.

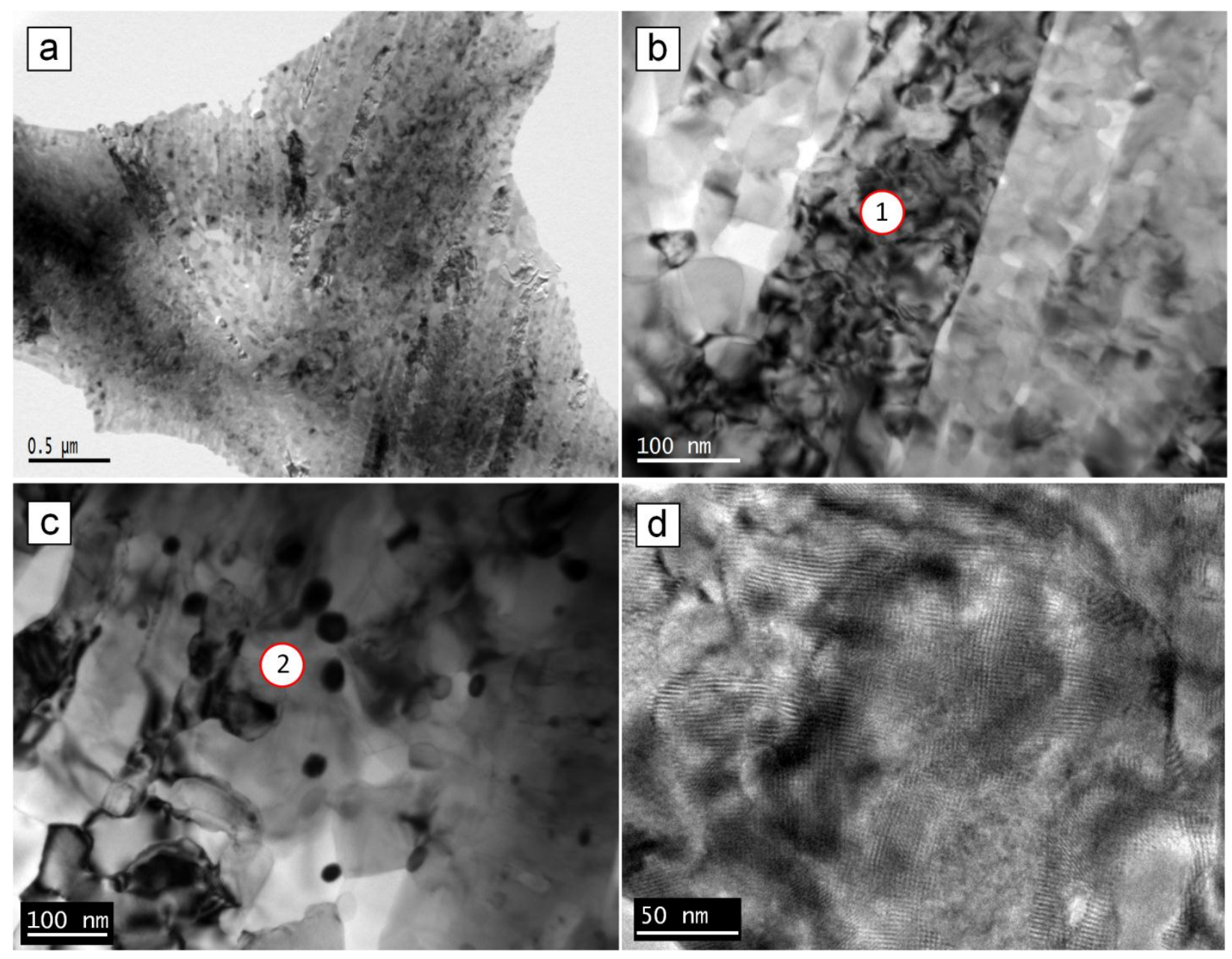

Figure 19. TEM micrographs showing ceramic regions affected by penetration of YSZ into the CCO grains.

Table 2. EDS data from points (1) and (2) in Figure 19 (b) and (c).

\begin{tabular}{|c|c|c|c|c|c|}
\hline & \multicolumn{5}{|c|}{ atomic \% } \\
\hline & $\mathrm{O}$ & $\mathrm{Ca}$ & $\mathrm{Co}$ & $\mathrm{Y}$ & $\mathrm{Zr}$ \\
\hline$(1)$ & 41.70 & 31.12 & 22.00 & 1.11 & 4.07 \\
\hline$(2)$ & 48.29 & 20.45 & 31.15 & 0.03 & 0.09 \\
\hline
\end{tabular}


$S, \rho$, and power factor of the CCO/YSZ composite are compared with the corresponding values of the pure CCO baseline in Figure 20. The $S$-T curve of the CCO/YSZ composite and the pure CCO baseline present similar behavior. Both curves correspond to p-type CCO (positive $S$ for the entire temperature range) and increase monotonically with temperature. The curves, as can be observed, present minor differences in magnitude: $S$ was improved below $560^{\circ} \mathrm{C}$ by the addition of YSZ, and reduced over this temperature. The improvement at room temperature was $4.0 \%$ while the reduction at $800^{\circ} \mathrm{C}$ was $2.3 \%$ meaning that $S$ was not substantially modified by the YSZ addition. This magnitude variation could be attributed to the doping action of $\mathrm{Zr}$ into the CCO lattice.

The effect of YSZ addition in the electrical resistivity of the CCO was also minor. Both the $\mathrm{CCO} / \mathrm{YSZ}$ and the baseline curves present oscillations and increase while increasing temperature over $500^{\circ} \mathrm{C}$. A favorable reduction in the resistivity magnitude can be observed for temperatures over $600^{\circ} \mathrm{C}$. The highest difference took place at $800^{\circ} \mathrm{C}$ where the resistivity decreased $11.0 \%$ respect to the baseline.

Finally, due to the minor variations registered for the $S$ and $\rho$ of the CCO after YSZ addition, no significant improvement of the power factor was obtained as can be observed in Figure 20. Only slight enhancement of the power factor can be appreciated below $460^{\circ} \mathrm{C}$ and above $730^{\circ} \mathrm{C}$. At $800^{\circ} \mathrm{C}$, the power factor improvement obtained was $7.1 \%$. 

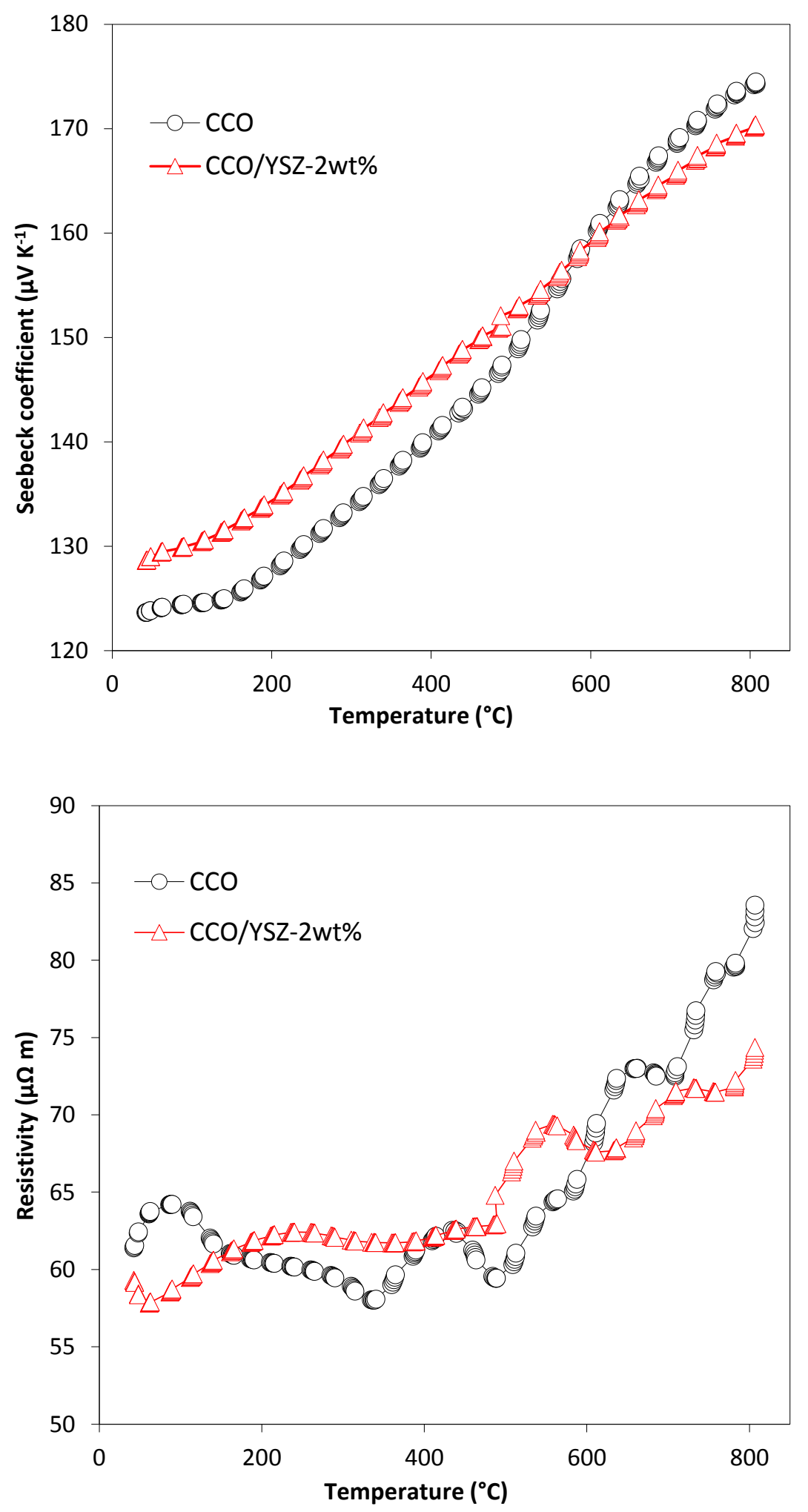


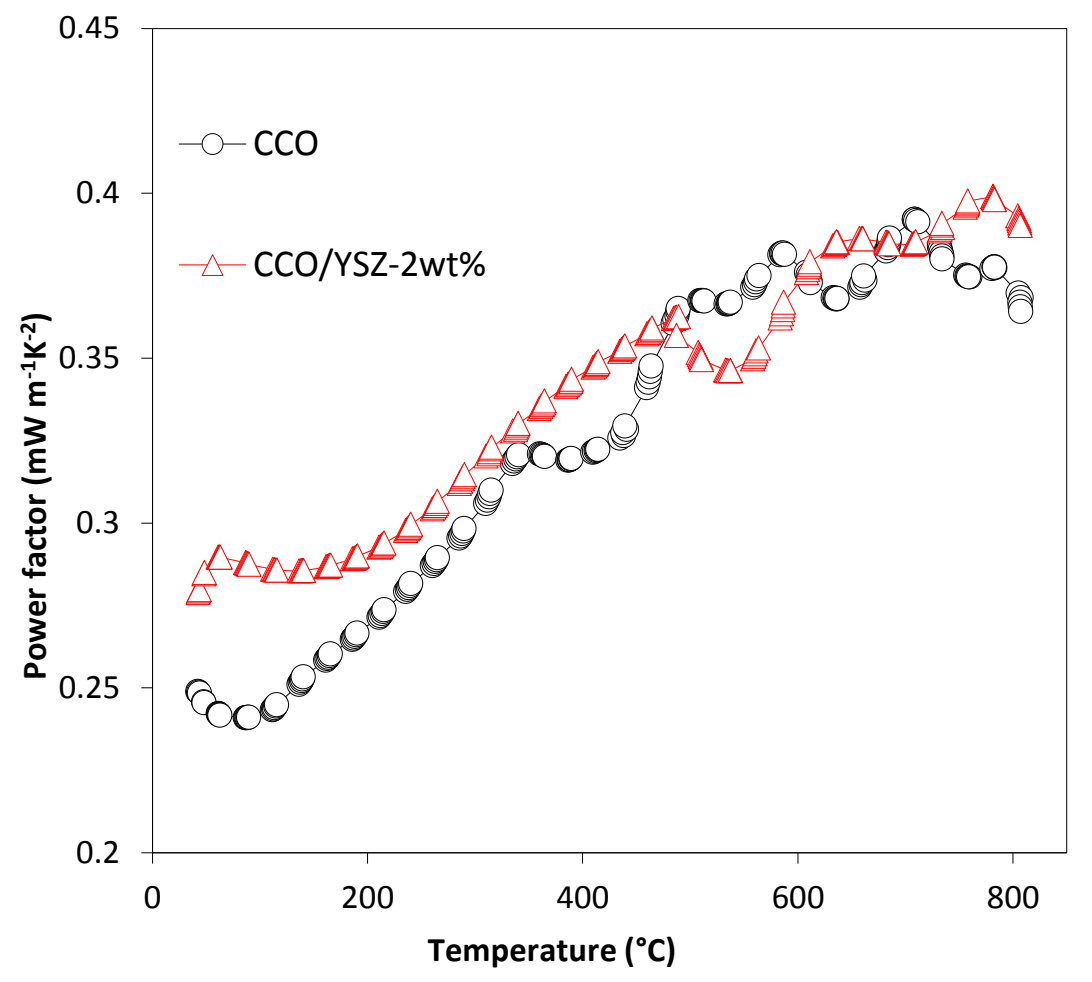

Figure 20. Seebeck coefficient, resistivity and power factor of the CCO/YSZ-2 wt $\%$ composite compared to the values for the pure $\mathrm{CCO}$ ceramic system.

\subsection{Effect of $\mathrm{CoAl}_{2} \mathrm{O}_{4}$ Addition on the Thermoelectric Properties of $\mathrm{Ca}_{3} \mathrm{Co}_{4} \mathrm{O}_{9}$ Ceramics}

\subsubsection{Experimental procedure}

The experimental $\mathrm{CCO} / \mathrm{CoAl}_{2} \mathrm{O}_{4}-10 \mathrm{wt} \%$ composite ceramic was produced by sol-gel method. The solution preparation process employed for obtaining the CCO powder was the same used previously for the synthesis of the pure $\mathrm{CCO}$ powder using deionized water as solvent. The particularity of this process was the addition of hydrothermal $\mathrm{CoAl}_{2} \mathrm{O}_{4}$ nanoparticles as the $\mathrm{CoAl}_{2} \mathrm{O}_{4}$ precursor instead of adding aluminum containing salts directly into the sol-gel solution. 
$\mathrm{CoAl}_{2} \mathrm{O}_{4}$ nanoparticles were prepared by hydrothermal synthesis method. Cobalt nitrate hexahydrate, aluminum nitrate nonahydrate and sodium hydroxide in stoichiometric ratios were dissolved and mixed in DEG. After obtaining a uniform solution, the mixture was placed into the hydrothermal reactor where the thermal cycle illustrated in Figure 21 was performed. A temperature of $230^{\circ} \mathrm{C}$ was applied for a dwell time of $48 \mathrm{~h}$. Reaction of the reagents occurred during this process to form a colloid of $\mathrm{CoAl}_{2} \mathrm{O}_{4}$ nanoparticles that were washed and collected by centrifugation.

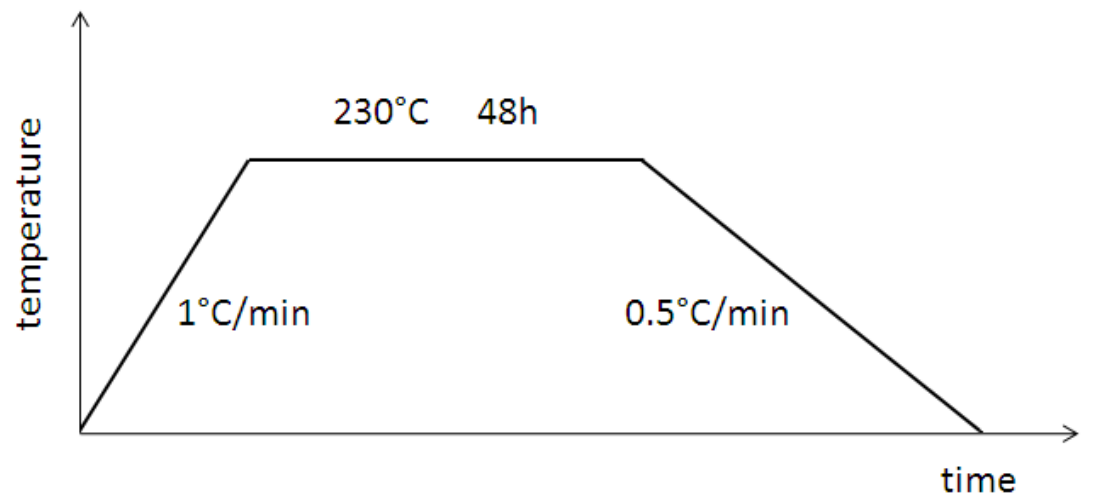

Figure 21. Temperature profile as a function of time applied during hydrothermal synthesis of the $\mathrm{CoAl}_{2} \mathrm{O}_{4}$ nanopowder.

$\mathrm{CoAl}_{2} \mathrm{O}_{4}$ nanoparticles were added to the $\mathrm{CCO}$ sol-gel solution just before stirring at $80^{\circ} \mathrm{C}$. The resulting solution was thoroughly mixed and stirred during 3 hours at $80^{\circ} \mathrm{C}$ and $200 \mathrm{rpm}$ until gelation. Then, the gel was dried in a box furnace at $500^{\circ} \mathrm{C}$ by two hours and the resultant ashes were subsequently grounded in a mortar by 20 minutes before performing calcination in a tube furnace with oxygen at $650^{\circ} \mathrm{C}$ for 4 hours. The obtained powders were consolidated into pellets by hot-pressing under $1 \mathrm{GPa}$ pressure at $150^{\circ} \mathrm{C}$ during 30 minutes followed by sintering in a box furnace during 18 hours at $920^{\circ} \mathrm{C}$. A flow diagram of the previously described 
procedure is presented in Figure 22. An additional pure CCO ceramic was prepared under the same conditions to serve as baseline for the $S, \rho$ and power factor measurements.

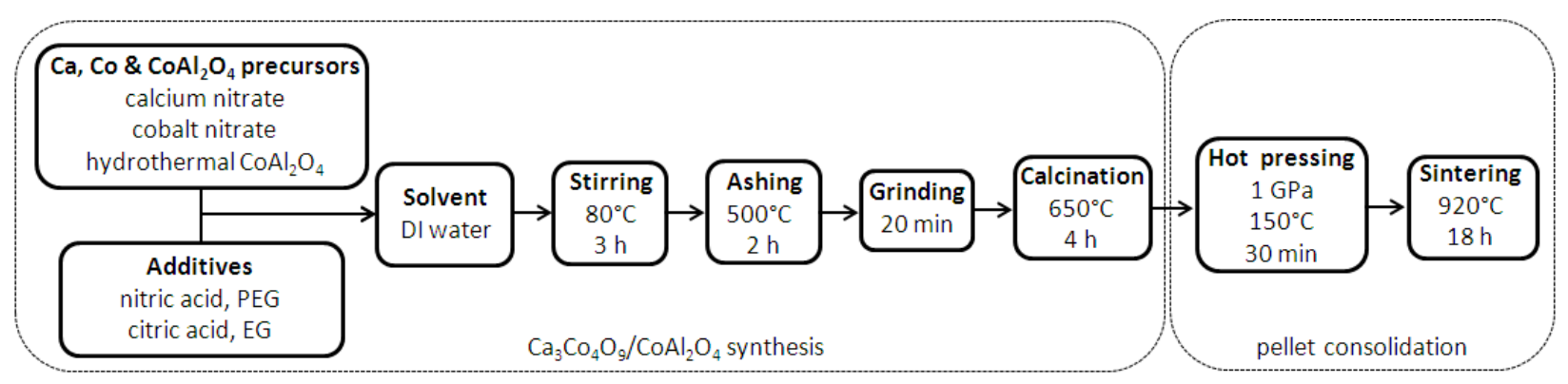

Figure 22. Flow diagram of the fabrication route of the $\mathrm{CCO} / \mathrm{CoAl}_{2} \mathrm{O}_{4}-10 \mathrm{wt} \%$ composite ceramic.

The pressed plane and fractured cross-section of the composite pellet were imaged using a JEOL JSM-7600F SEM. TEM analyses of the hydrothermal $\mathrm{CoAl}_{2} \mathrm{O}_{4}$ nanopowder and composite pellet were performed using a JEOL TEM-2100 operating at $200 \mathrm{kV}$ equipped with EDS analyzer. The TEM specimens were prepared by conventional polishing of the samples using abrasive films. Indexing of the electron diffraction patterns was assisted by the ICDD PDF-4+ version 4.1203 software. Finally, a Linseis LSR-1100 unit was employed to simultaneously measure $S, \rho$ and power factor of both the pure $\mathrm{CCO}$ ceramic and the $\mathrm{CCO} / \mathrm{CoAl}_{2} \mathrm{O}_{4}-10 \mathrm{wt} \%$ composite. Measurements were performed in the direction parallel to the pressed plane from room temperature up to $800^{\circ} \mathrm{C}$ in a He environment.

\subsubsection{Results and discussion}

TEM analysis was performed on the $\mathrm{CoAl}_{2} \mathrm{O}_{4}$ nanoparticles prepared by hydrothermal synthesis method. The TEM micrograph in Figure 23 (a) shows the highly fine and uniform nanoparticles obtained with spherical shape and size around $5 \mathrm{~nm}$ while Figure 23 Figure 23(b) displays the obtained electron diffraction ring pattern corresponding to the cubic $\mathrm{CoAl}_{2} \mathrm{O}_{4}$ phase. 
Representative EDS data from the nanopowder is presented in Table 3. The atomic elemental composition obtained corresponds to that of $\mathrm{CoAl}_{2} \mathrm{O}_{4}$.
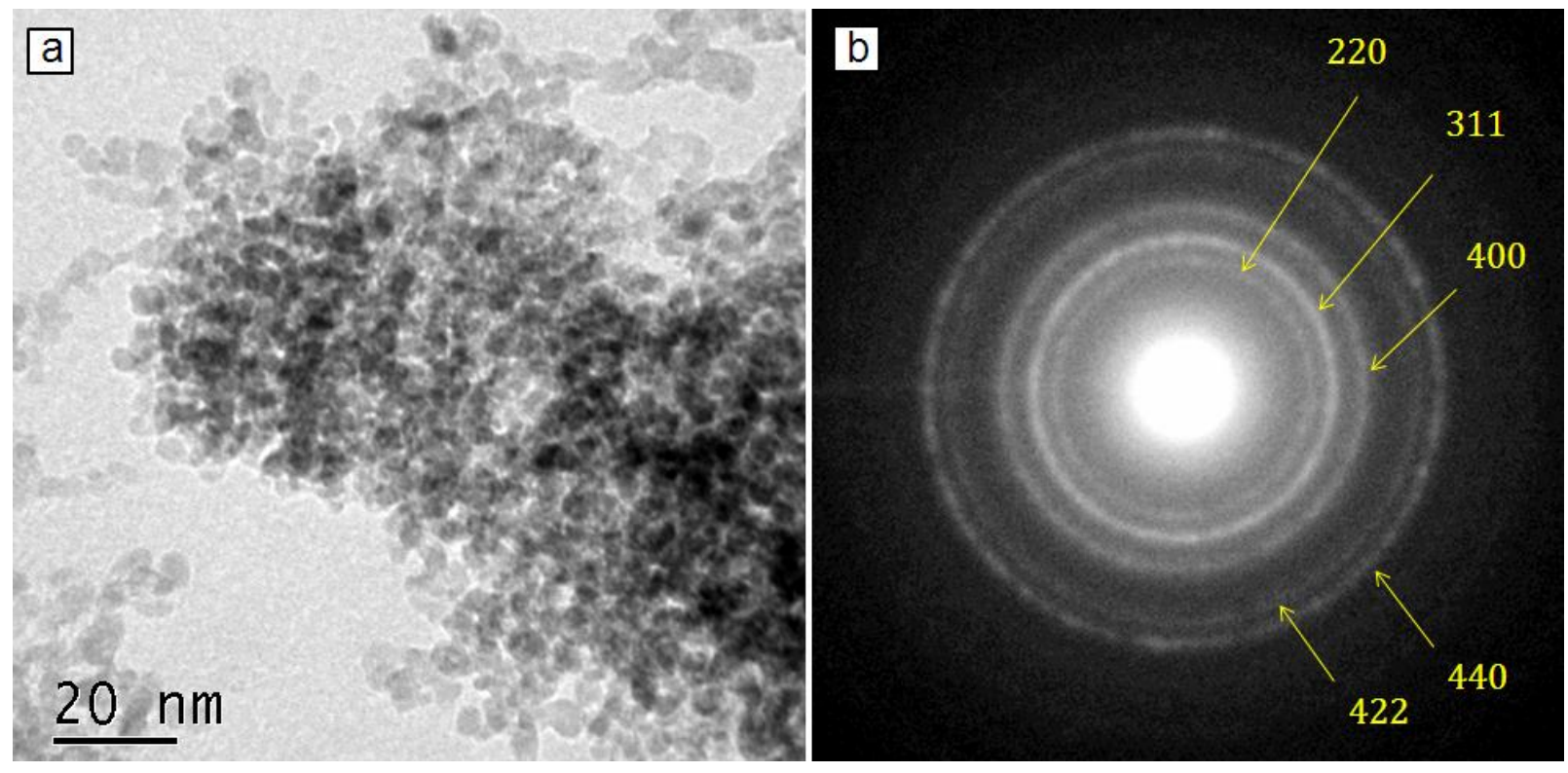

Figure 23. TEM micrographs of the hydrothermal $\mathrm{CoAl}_{2} \mathrm{O}_{4}$ nanopowder synthesized: (a) bright field image and (b) selected area electron diffraction pattern.

Table 3. Representative EDS data from the $\mathrm{CoAl}_{2} \mathrm{O}_{4}$ nanoparticles.

\begin{tabular}{|c|c|c|}
\hline \multicolumn{3}{|c|}{ atomic \% } \\
\hline $\mathrm{O}$ & $\mathrm{Al}$ & $\mathrm{Co}$ \\
\hline 59.42 & 27.91 & 12.67 \\
\hline
\end{tabular}

SEM micrographs of the pressed plane and fractured cross-section of the prepared $\mathrm{CCO} / \mathrm{CoAl}_{2} \mathrm{O}_{4}$ ceramic are presented in Figure 24. This specimen presents platelet-shaped grains, high porosity and a poor grain alignment after performing hot-pressing and sintering. 

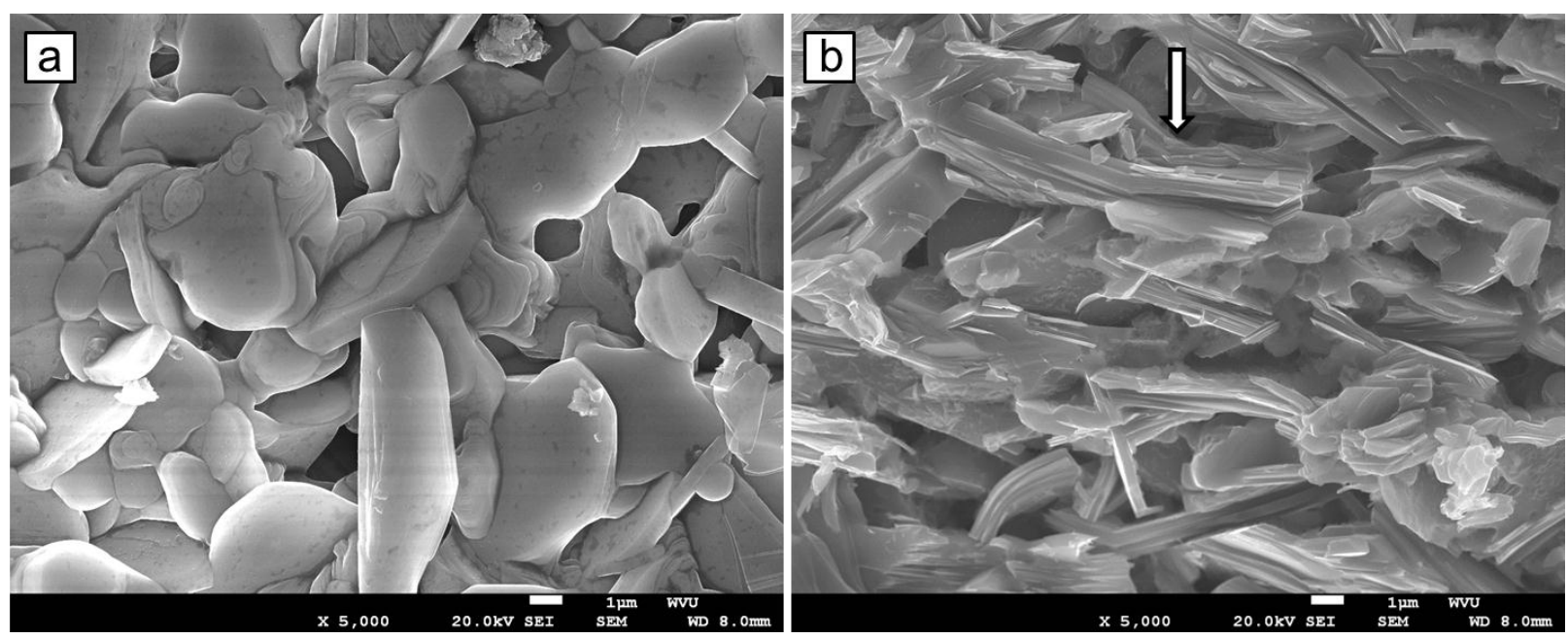

Figure 24. SEM micrographs of the $\mathrm{CCO} / \mathrm{CoAl}_{2} \mathrm{O}_{4}$ composite ceramic showing the morphology of: (a) the pressed plane and (b) a fractured cross-section surface. The arrow indicates the applied stress direction.

TEM analysis of the $\mathrm{CCO} / \mathrm{CoAl}_{2} \mathrm{O}_{4}$ composite allowed observing the structure of the ceramic at the nano-scale. The prepared composite ceramic presents the typical layered structure composed by bundles of nano-lamellas as detailed in Figure 25 (a). EDS analysis of several regions revealed the existence of $\mathrm{Al}$ ions within the $\mathrm{CCO}$ crystal structure as doping agent (see Table 4). Average concentration of $\mathrm{Al}$ within the $\mathrm{CCO}$ was found to be around 1.4 atomic $\%$. The ceramic was also found to present crystalline cobalt oxide grains distributed throughout the microstructure as the one observed in Figure 25 (b). These grains possess irregular shapes and a wide size distribution (with size ranging from a fraction of micron to several microns). From the EDS data in Table 4 and the electron diffraction pattern in Figure 25 (c) it was determined that cobalt oxide grain present cubic $\mathrm{Co}_{3} \mathrm{O}_{4}$ structure. As mentioned before, the formation of small amounts of this phase is common in the CCO ceramics [14]. 

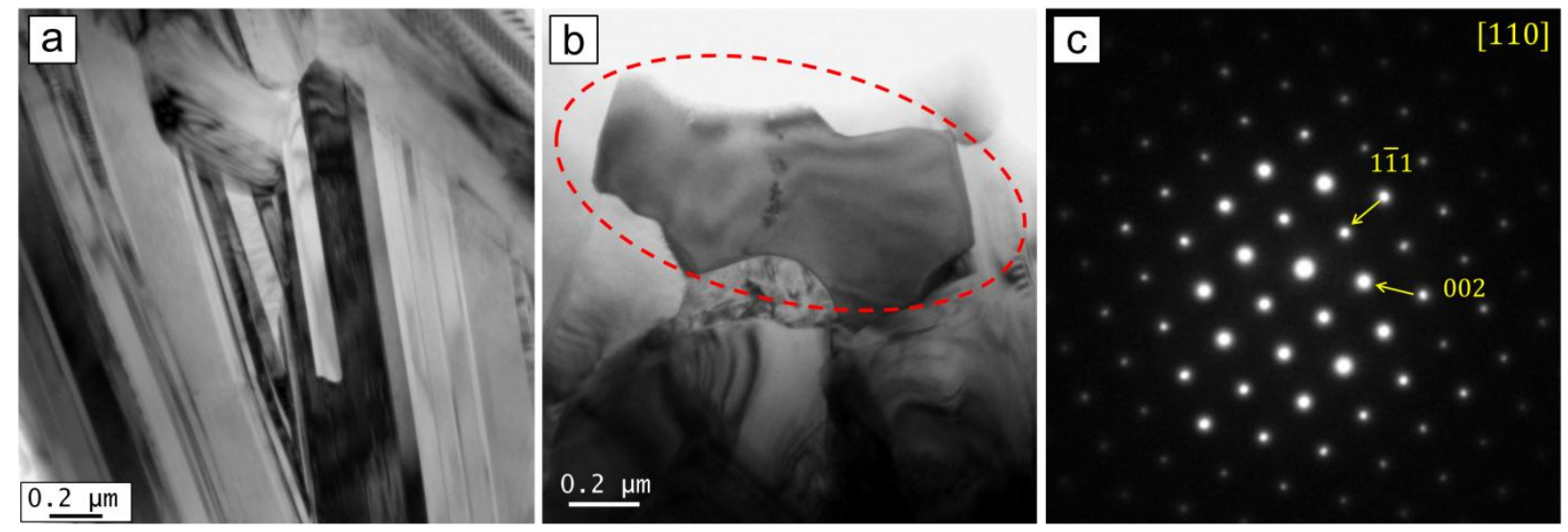

Figure 25. TEM micrographs showing: (a) the layered structure of the $\mathrm{CCO}$ and (b) $\mathrm{a} \mathrm{Co}_{3} \mathrm{O}_{4}$ grain with its respective diffraction pattern (c).

Table 4. Representative EDS data from the CCO and cobalt oxide grain presented in Figure 25

(a) and (b) respectively.

\begin{tabular}{|c|c|c|c|c|}
\hline & \multicolumn{4}{|c|}{ atomic \% } \\
\hline & O & Al & Ca & Co \\
\hline (a) & 52.91 & 1.44 & 20.11 & 25.55 \\
\hline (b) & 59.46 & 1.34 & - & 39.19 \\
\hline
\end{tabular}

Additional features were observed during the TEM survey, principally the occurrence of an amorphous circular phase composed primarily by $\mathrm{Al}$ and $\mathrm{O}$ and located within the $\mathrm{CCO}$ grains. These features were observed throughout the ceramic microstructure. The amorphous phase is presented in the TEM micrographs in Figure 26. Representative EDS data from these amorphous regions is presented in Table 5; minor Ca concentration was also detected within. 

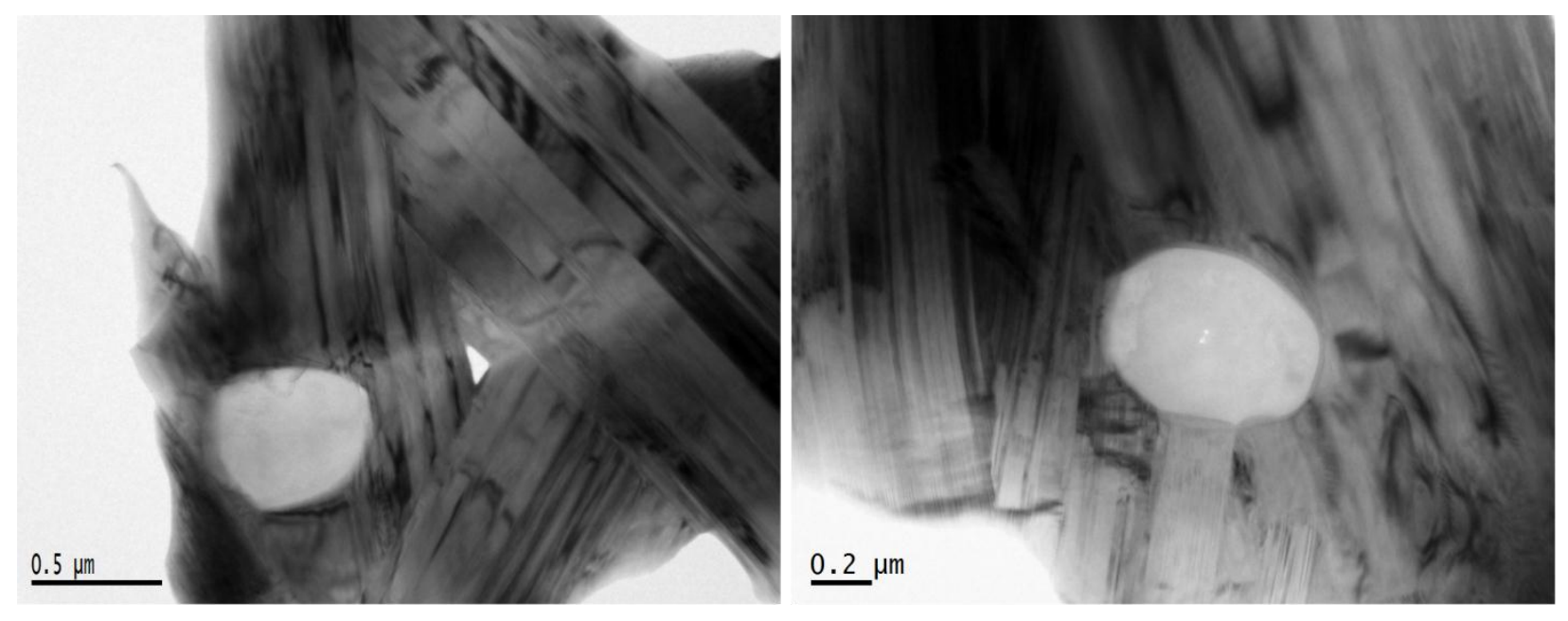

Figure 26. TEM micrographs showing the amorphous Al-O phase found within the CCO grains.

Table 5. Representative EDS data from the Al-O amorphous phase imaged in Figure 26.

\begin{tabular}{|c|c|c|}
\hline \multicolumn{3}{|c|}{ atomic \% } \\
\hline $\mathrm{O}$ & $\mathrm{Al}$ & $\mathrm{Ca}$ \\
\hline 59.40 & 36.58 & 4.02 \\
\hline
\end{tabular}

Seebeck coefficient, resistivity and power factor of the experimental $\mathrm{CCO} / \mathrm{CoAl}_{2} \mathrm{O}_{4}$ alloy are presented in Figure 27 along with the baseline data (pure CCO). A significant reduction of $S$ at low temperature resulted from the addition of $\mathrm{CoAl}_{2} \mathrm{O}_{4}$ to the $\mathrm{CCO}$ as can be observed in the $S-T$ curve. At low temperature, a $30.7 \%$ reduction occurred. At $800^{\circ} \mathrm{C}$, in contrast, the two curves present identical magnitudes. The electrical resistivity was also affected by the $\mathrm{CoAl}_{2} \mathrm{O}_{4}$ addition; especially at high temperature were $\rho$ of the alloyed sample doubles the value of the baseline. The behavior of the resistivity curve changed from semiconducting-like to metallic-like behavior. As a consequence of the $S$ reduction and the most critical $\rho$ increment, the power factor was severely deteriorated by the addition of $\mathrm{CoAl}_{2} \mathrm{O}_{4}$. At high temperature the power factor was reduced to half of the value of the baseline. 

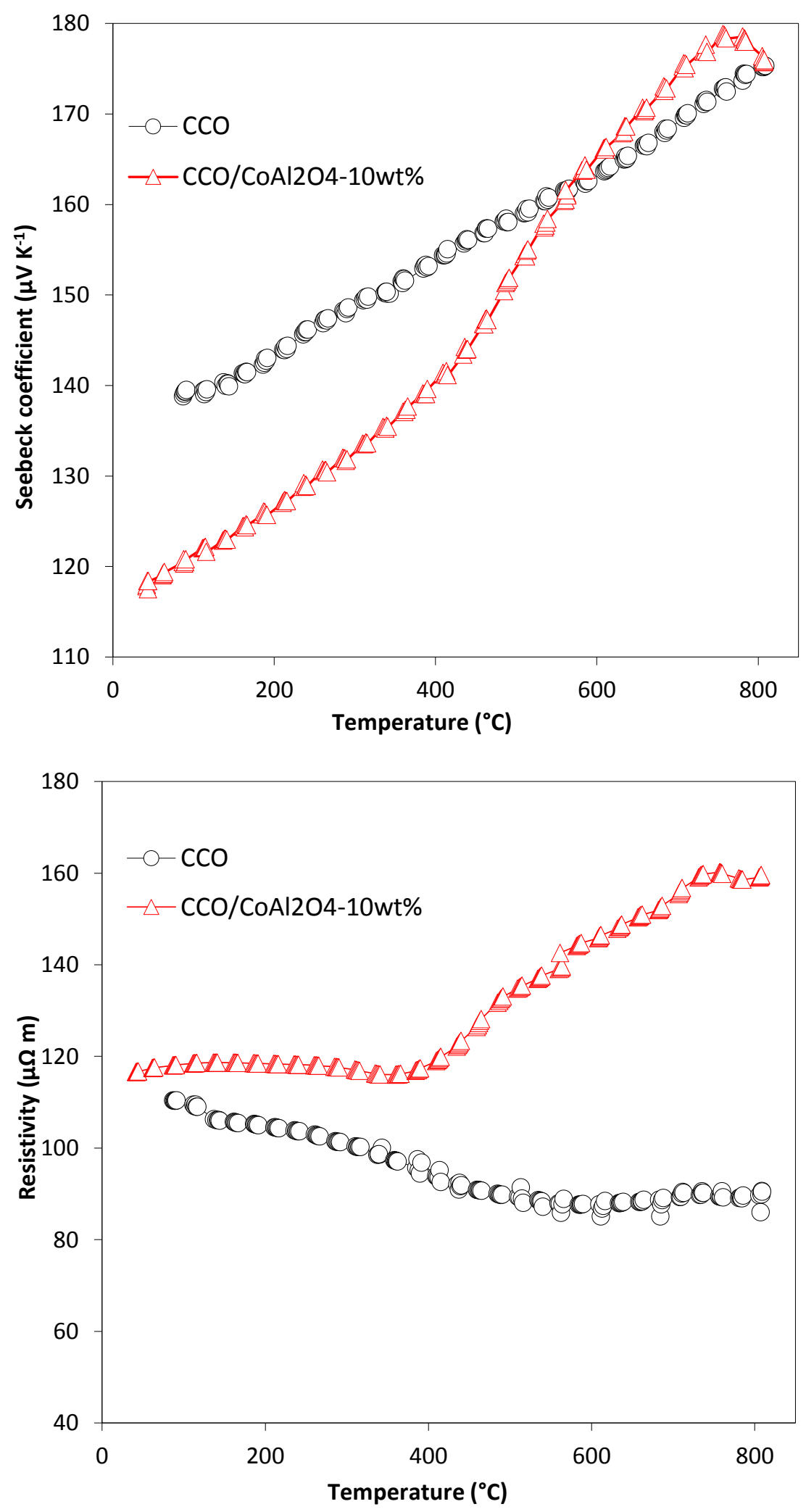


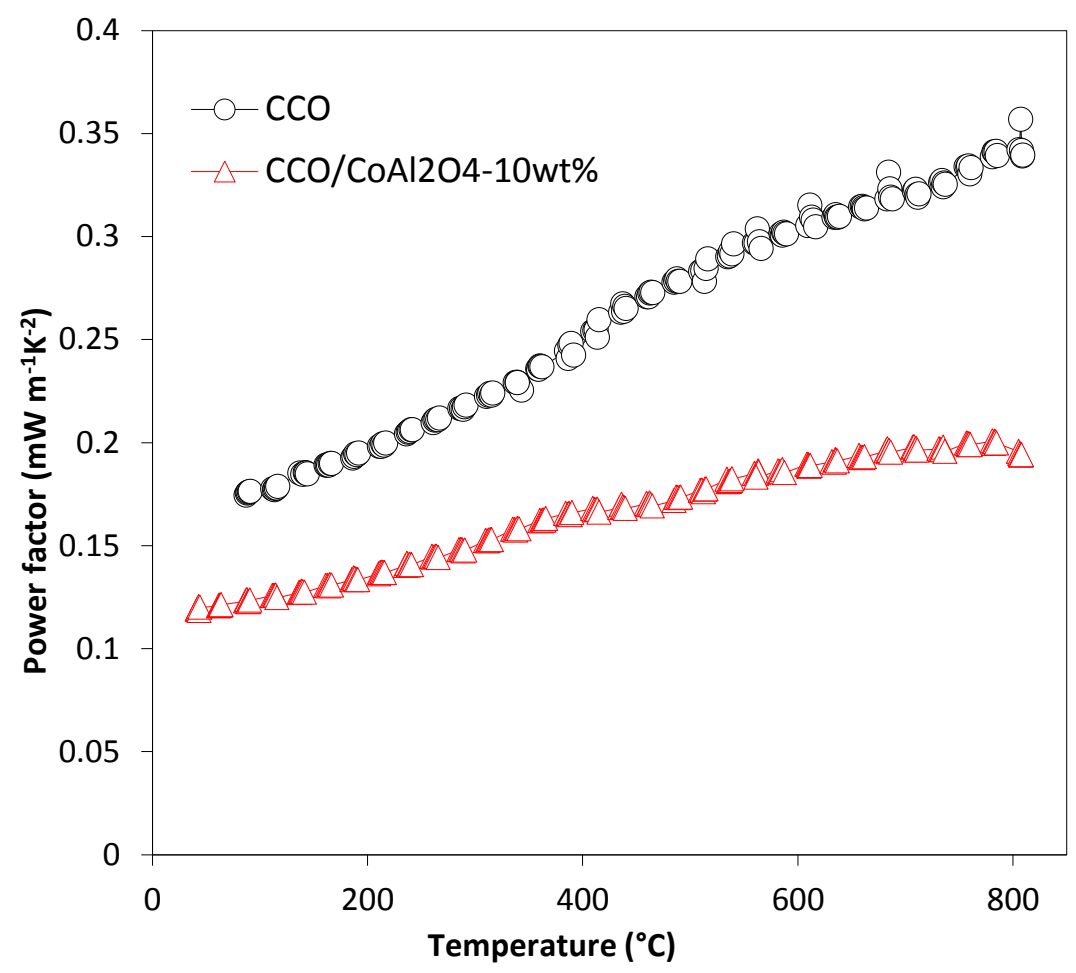

Figure 27. Seebeck coefficient, resistivity and power factor of the experimental $\mathrm{CCO} / \mathrm{CoAl}_{2} \mathrm{O}_{4}$ alloy compared with the baseline.

\subsection{Effect of $\mathrm{NiFe}_{2} \mathrm{O}_{4}$ Addition on the Thermoelectric Properties of $\mathrm{Ca}_{3} \mathrm{Co}_{4} \mathrm{O}_{9}$ Ceramics}

\subsubsection{Experimental procedure}

The experimental $\mathrm{Ca}_{3} \mathrm{Co}_{4} \mathrm{O}_{9} / \mathrm{NiFe}_{2} \mathrm{O}_{4}-2 \mathrm{wt} \%$ composite ceramic was synthesized by the solgel route with the addition of $\mathrm{NiFe}_{2} \mathrm{O}_{4}$ nanoparticles during solution preparation. The procedure employed for the sol-gel preparation was the same used in the synthesis of the pure $\mathrm{Ca}_{3} \mathrm{Co}_{4} \mathrm{O}_{9}$ system using deionized water as solvent, plus the addition $\mathrm{NiFe}_{2} \mathrm{O}_{4}$ nanopowder as the alloying agent. The resultant sol-gel solution was processed until pellet consolidation by following the route summarized in Figure 28. 


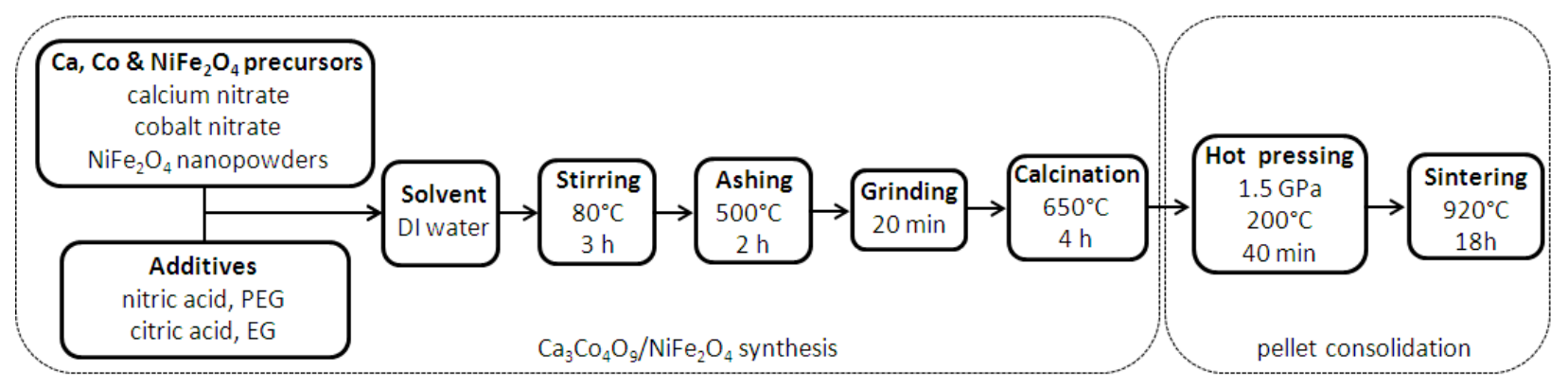

Figure 28. Flow diagram of the fabrication route of the $\mathrm{Ca}_{3} \mathrm{Co}_{4} \mathrm{O}_{9} / \mathrm{NiFe}_{2} \mathrm{O}_{4}-2$ wt $\%$ composite ceramic.

A JEOL JSM-7600F microscope was employed for SEM analysis of the pressed plane and fractured cross-section of the composite pellet. TEM analysis of the composite ceramic was also performed using a JEOL TEM-2100 equipped with EDS analyzer. The TEM specimen was prepared by conventional polishing of the samples using abrasive films. Indexing of the electron diffraction patterns was assisted by the ICDD PDF-4+ version 4.1203 software. Additionally, $S$, $\rho$ and power factor of the ceramics were measured with a Linseis LSR-1100 unit in the direction parallel to the pressed plane from room temperature up to $800^{\circ} \mathrm{C}$ in a $\mathrm{He}$ environment. The baseline data used for the CCO/YSZ-2 wt\% analysis was also used in this section since the samples were prepared following identical experimental procedures.

\subsubsection{Results and discussion}

SEM images of the pressed plane and fractured cross-section of the prepared $\mathrm{CCO} / \mathrm{NiFe}_{2} \mathrm{O}_{4}$ ceramic are presented in Figure 29. This specimen presents high porosity and a poor texture after performing hot-pressing and sintering operations. Ceramic grains present platelet shape with size ranging from 1 to $10 \mu \mathrm{m}$. 

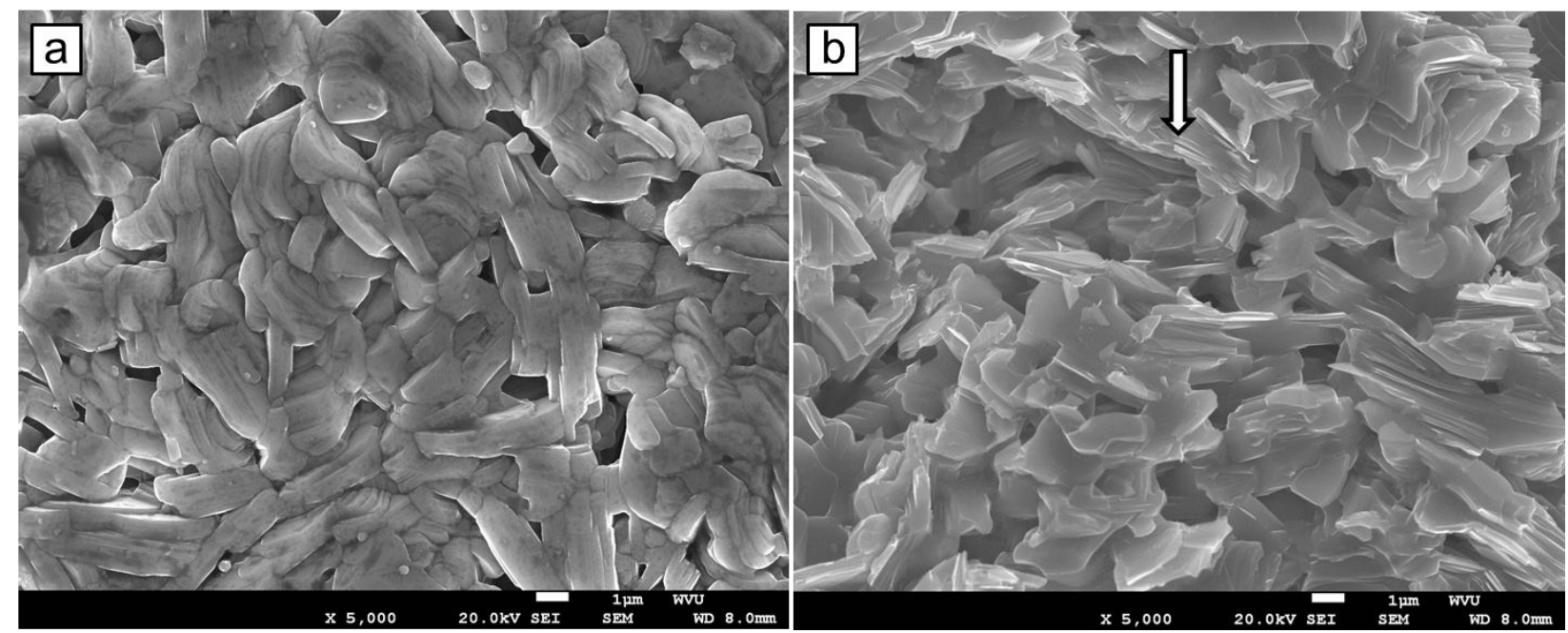

Figure 29. SEM micrographs of the $\mathrm{CCO} / \mathrm{NiFe}_{2} \mathrm{O}_{4}-2 \mathrm{wt} \%$ composite ceramic showing the morphology of: (a) the pressed plane and (b) a fractured cross-section surface. The arrow indicates the applied stress direction.

TEM micrographs of the $\mathrm{CCO} / \mathrm{NiFe}_{2} \mathrm{O}_{4}$ ceramic nanostructure are presented in Figure 30. It can be observed that ceramic grains are constituted by layers of $\mathrm{CCO}$ nano-lamellas having parallel $c$-axis. The electron diffraction pattern presented in Figure 30 (c) corresponds to the [110] zone axis of the CCO system and shows that the direction of $c$-axis is perpendicular to the largest dimension of nano-lamellas. EDS analysis from composite ceramic reveals the existence of Fe as doping agent in the $\mathrm{CCO}$ as indicated in Table 6. Fe concentration throughout the specimen was found to be around 1.5 atomic $\%$. The substitution of $\mathrm{Co}^{3+}$ by $\mathrm{Fe}^{2+}$ is known to increase the hole carrier concentration of the CCO leading to the reduction of $\rho$ and the improvement of the $S$ at room temperature $[11,17]$. 

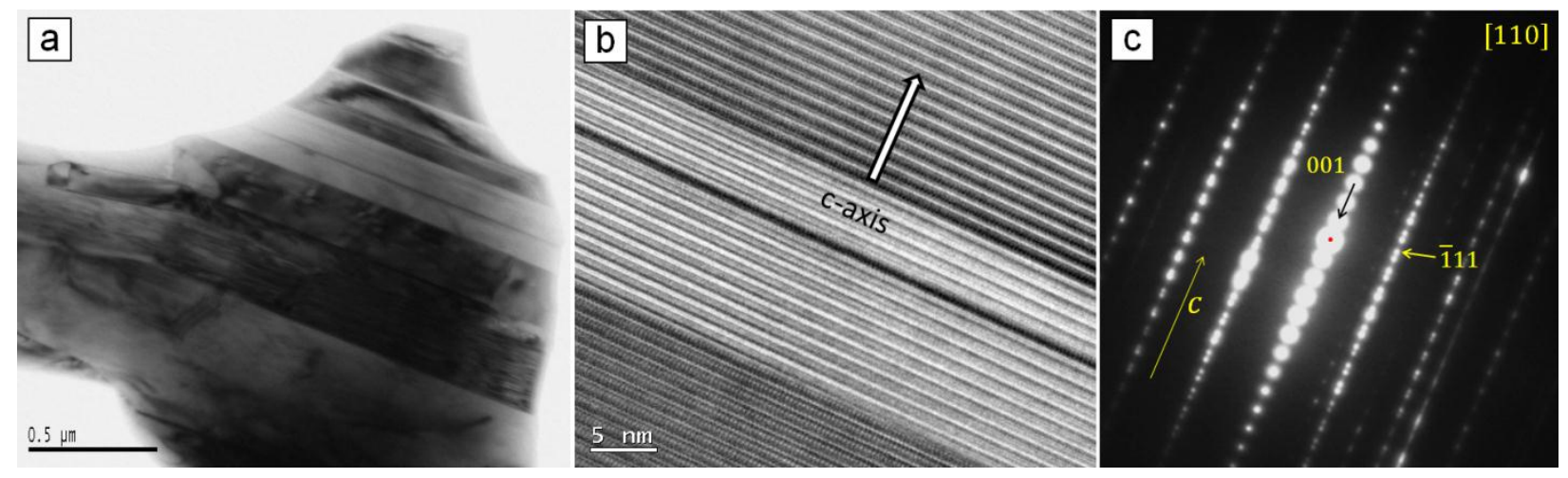

Figure 30. TEM micrographs from the $\mathrm{CCO} / \mathrm{NiFe}_{2} \mathrm{O}_{4}$ composite: (a) bright field image, (b)

HRTEM image showing the atomic planes and nano-lamellas and (c) its respective electron diffraction pattern. The direction of the $c$-axis is indicated.

Table 6. Representative EDS data from the $\mathrm{CCO} / \mathrm{NiFe}_{2} \mathrm{O}_{4}$ ceramic.

\begin{tabular}{|c|c|c|c|}
\hline \multicolumn{4}{|c|}{ atomic \% } \\
\hline $\mathrm{O}$ & $\mathrm{Ca}$ & $\mathrm{Co}$ & $\mathrm{Fe}$ \\
\hline 58.34 & 16.90 & 23.63 & 1.13 \\
\hline
\end{tabular}

Different crystal phases were found along with the $\mathrm{CCO}$ in the composite microstructure. That is the case of large cobalt oxide grains located throughout the composite ceramic. Figure 31 (a) and (c) present a $\mathrm{Co}_{3} \mathrm{O}_{4}$ grain with its respective electron diffraction pattern corresponding to the [110] zone axis. Nickel containing phases were also observed in the ceramic; Figure 31 (b) and (d) present an image of a cobalt nickel oxide with its respective electron diffraction pattern. This phase was identified as the cubic $\mathrm{Co}_{0.5} \mathrm{Ni}_{0.5} \mathrm{O}$ crystal system. EDS data from both the $\mathrm{Co}_{3} \mathrm{O}_{4}$ and $\mathrm{Co}_{0.5} \mathrm{Ni}_{0.5} \mathrm{O}$ phases is summarized in Table 7. 

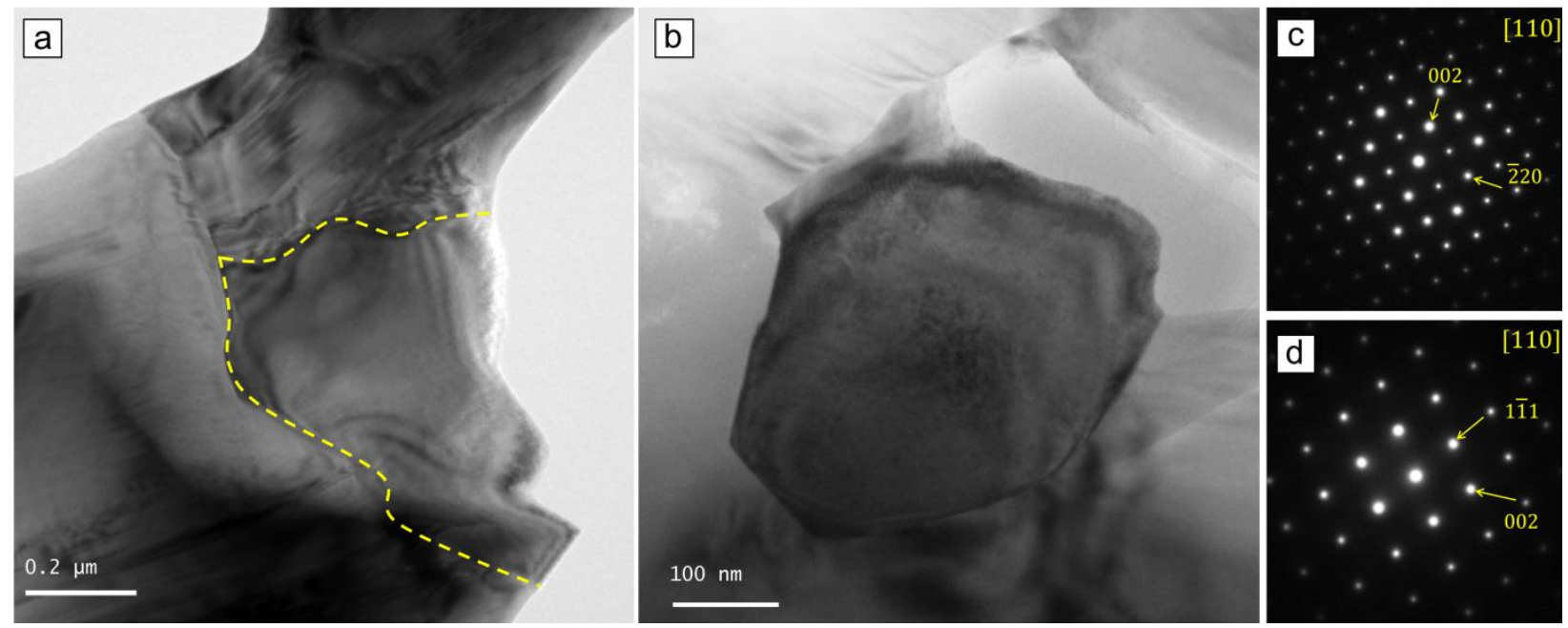

Figure 31. TEM micrographs and diffraction patterns from: (a) and (c) the $\mathrm{Co}_{3} \mathrm{O}_{4}$ phase, and (b) and (d) the $\mathrm{Co}_{0.5} \mathrm{Ni}_{0.5} \mathrm{O}$ phase.

Another cobalt oxide phase was observed in the $\mathrm{CCO} / \mathrm{NiFe}_{2} \mathrm{O}_{4}$ composite and imaged in Figure 32 (a). It was identified as the cubic $\mathrm{Co}_{2} \mathrm{NiO}_{4}$ crystal system by electron diffraction and EDS analyses. Figure 32 (b) presents the electron diffraction pattern corresponding to the [011] zone axis and the EDS data is reported in Table 8. These crystal particles with size ranging from 100 to $500 \mathrm{~nm}$ were found to be dispersed throughout the specimen.

Table 7. EDS data from the grains presented in Figure 31 (a) and (b).

\begin{tabular}{|c|c|c|c|c|}
\hline \multicolumn{5}{|c|}{ atomic \% } \\
\hline & O & $\mathrm{Ca}$ & $\mathrm{Co}$ & $\mathrm{Ni}$ \\
\hline (a) & 56.50 & 0.43 & 43.07 & - \\
\hline (b) & 41.76 & 0.68 & 28.50 & 29.06 \\
\hline
\end{tabular}




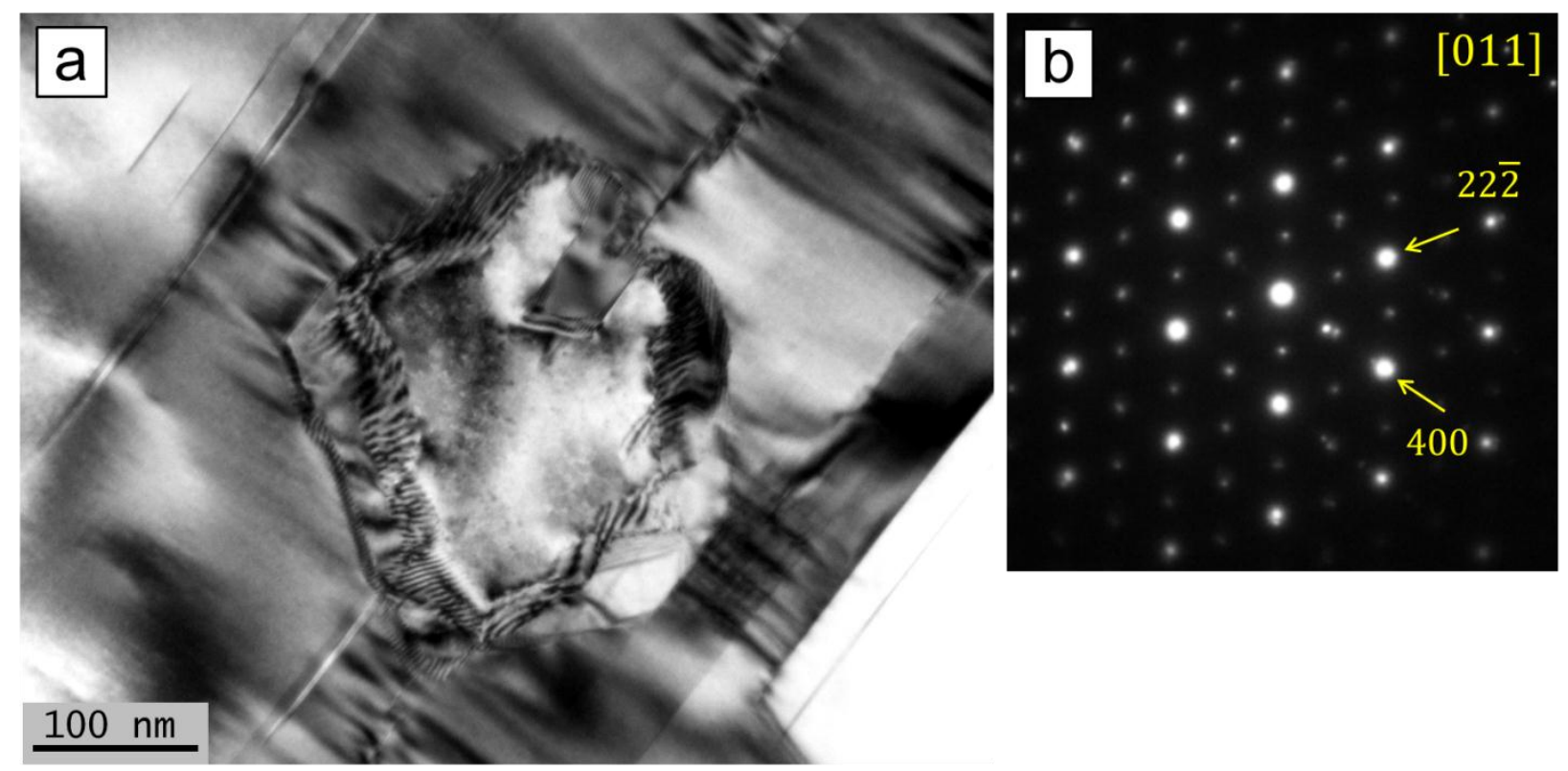

Figure 32. (a) TEM micrograph from a $\mathrm{Co}_{2} \mathrm{NiO}_{4}$ grain located within the $\mathrm{CCO}$ and its respective electron diffraction pattern (b) corresponding to the [011] zone axis.

Table 8. EDS data from the nanoparticle in Figure 32.

\begin{tabular}{|c|c|c|}
\hline \multicolumn{3}{|c|}{ atomic \% } \\
\hline $\mathrm{O}$ & $\mathrm{Co}$ & $\mathrm{Ni}$ \\
\hline 49.63 & 31.56 & 18.81 \\
\hline
\end{tabular}

Figure 33 presents the measured $S, \rho$ and power factor of the $\mathrm{CCO} / \mathrm{NiFe}_{2} \mathrm{O}_{4}$ alloy compared with the respective baseline data from the pure $\mathrm{CCO}$ ceramic. It can be appreciated that the $S-T$ curve of the $\mathrm{CCO} / \mathrm{NiFe}_{2} \mathrm{O}_{4}$ composite behaves identically to the baseline, increasing monotonically throughout the entire temperature range with equivalent magnitude values. Both curves indicate that the two ceramics are p-type oxides.

For the case of the electrical resistivity, the addition of $\mathrm{NiFe}_{2} \mathrm{O}_{4}$ resulted in a significant magnitude increment respect to the baseline, meaning that the electrical conductivity of the $\mathrm{CCO}$ 
was severely deteriorated. The resistivity of the composite sample increased $79.0 \%$ in average respect to the baseline and $82.7 \%$ at $800^{\circ} \mathrm{C}$. Despite of the magnitude variation, the $\rho$ - $T$ curves present the same behavior, where $\rho$ increases as temperature does.

As the result of the $\rho$ increment, the power factor of the composite sample is inferior to that of the baseline; an average reduction of $44.3 \%$ for the entire temperature range was obtained and $48.2 \%$ at $800^{\circ} \mathrm{C}$. The previous results clearly expose that the resistivity (and consequently the power factor) of the CCO ceramic is severely affected by the addition of $2 \mathrm{wt} \% \mathrm{NiFe}_{2} \mathrm{O}_{4}$. This effect is negative in terms of thermoelectric performance due to the significant deterioration of the electrical conductivity and power factor of the ceramic system.

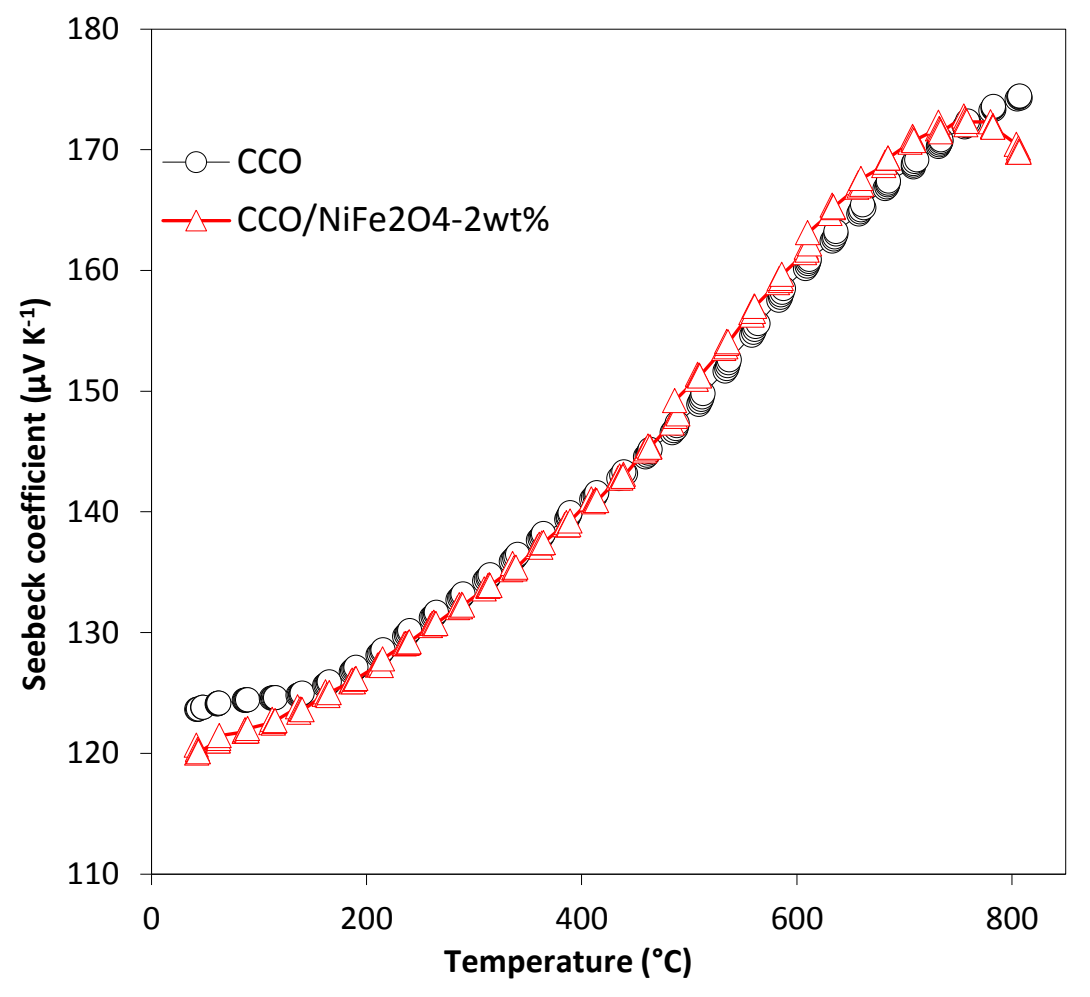



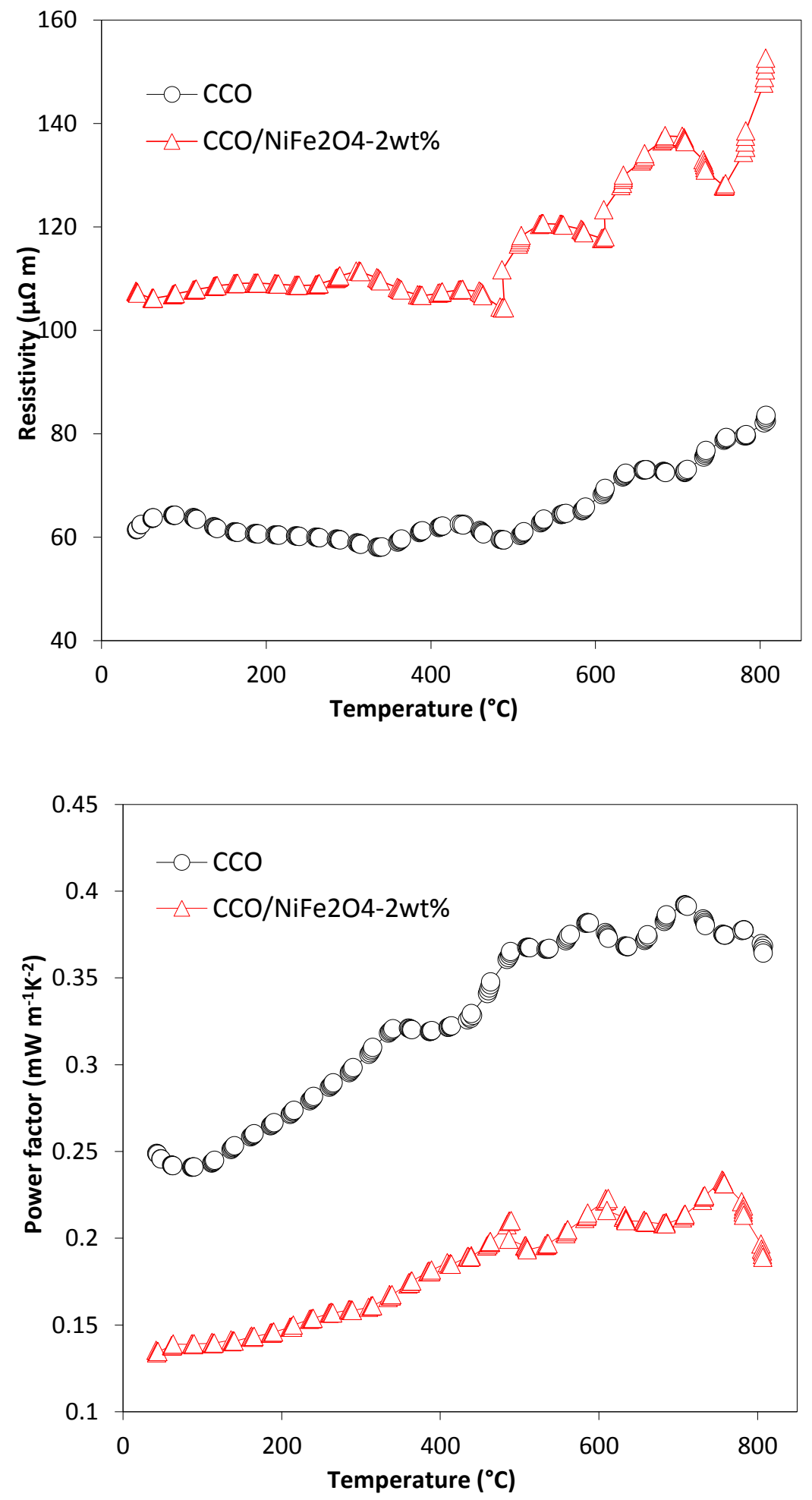

Figure 33. Seebeck coefficient, resistivity and power factor curves of the $\mathrm{Ca}_{3} \mathrm{Co}_{4} \mathrm{O}_{9} / \mathrm{NiFe}_{2} \mathrm{O}_{4}-2$ $\mathrm{wt} \%$ composite compared to the baseline data. 


\title{
CHAPTER 5: EFFECT OF THE ADDITION OF PRECIOUS METALS ON THE THERMOELECTRIC PROPERTIES OF
}

\author{
$\mathrm{Ca}_{3} \mathrm{Co}_{4} \mathrm{O}_{9}$ CERAMICS
}

\subsection{Effect of Pd doping on the thermoelectric properties of $\mathrm{Ca}_{3} \mathrm{Co}_{4} \mathrm{O}_{9}$ \\ Ceramics}

\subsubsection{Experimental procedure}

Precursor powders with nominal composition $\mathrm{Ca}_{3} \mathrm{Co}_{4-\mathrm{x}} \mathrm{Pd}_{\mathrm{x}} \mathrm{O}_{9}(\mathrm{x}=0,0.01$ and 0.05$)$ were prepared by sol-gel route followed by hot pressing and sintering processes. For the synthesis of these ceramic systems, the same process used to prepare the pure CCO powder was employed. The only difference was the addition of palladium chloride $\left(\mathrm{PdCl}_{2}\right)$ during the solution preparation before stirring at $80^{\circ} \mathrm{C}$. Precise amounts of $\mathrm{PdCl}_{2}$ were added for the synthesis of the $\mathrm{x}=0.01$ and $\mathrm{x}=0.05$ systems. The resultant solutions were stirred until gelation and ashed. The products were grounded, calcined, hot-pressed and sintered. The flow diagram in Figure 34 summarizes the fabrication of the samples. An additional pure $\mathrm{CCO}$ ceramic $(\mathrm{x}=0)$ was prepared under the same conditions to serve as baseline for the $S, \rho$ and power factor measurements.

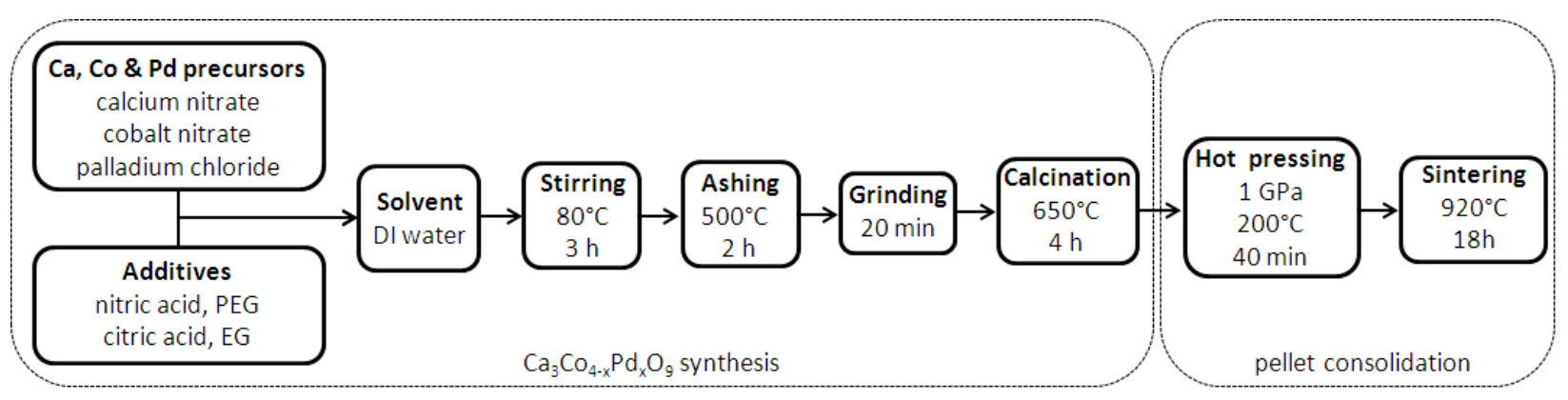

Figure 34. Fabrication route for the $\mathrm{Ca}_{3} \mathrm{Co}_{4-\mathrm{x}} \mathrm{Pd}_{\mathrm{x}} \mathrm{O}_{9}(\mathrm{x}=0,0.01$ and 0.05$)$ ceramics. 
Pressed plane and cross-sectional fractured surfaces of the consolidated $\mathrm{Ca}_{3} \mathrm{Co}_{4-\mathrm{x}} \mathrm{Pd}_{\mathrm{x}} \mathrm{O}_{9}$ $(\mathrm{x}=0.01$ and 0.05$)$ ceramics were imaged by SEM using a JEOL JSM-7600F. TEM analysis of the ceramics was also performed using a JEOL TEM-2100 coupled with EDS analyzer. Indexing of the electron diffraction patterns was assisted by the ICDD PDF-4+ version 4.1203 software. TEM specimens were prepared by conventional polishing of the samples using abrasive films. $S$, $\rho$ and power factor measurements were performed in the direction parallel to the pressed plane from room temperature up to $800^{\circ} \mathrm{C}$ using a Linseis LSR-1100 equipment in a He environment.

\subsubsection{Results and discussion}

SEM micrographs from two different surfaces: pressed plane and fractured cross-section of the $x=0.01$ and 0.05 ceramic systems are presented in Figure 35 and Figure 36 respectively. The pressed plane and cross-sectional images are set side-by-side to show the effect of the uniaxial pressing applied during consolidation. The different morphologies observed for the two surfaces indicate that a textured ceramic was obtained.

The microstructure of the $\mathrm{x}=0.01$ ceramic was observed to be composed by a mixture of two types of grains: short flake-like grains with lengths under $10 \mu \mathrm{m}$, and large and elongated platelet-shaped grains with lengths up to $200 \mu \mathrm{m}$. The large platelet-shaped grains were not observed in the microstructure of the CCO ceramic samples analyzed previously. Both small and large grains tend to be oriented perpendicular to the stress axis. The alignment, however, is not total since several grains diverge from the intended grain direction.

SEM micrographs from the $\mathrm{x}=0.05$ sample show that the ceramic is composed by small platelet-shaped grains ranging in size between 1 to $10 \mu \mathrm{m}$; alongside to larger, elongated and irregular grains with lengths surpassing $50 \mu \mathrm{m}$. Compared with the previous sample, the larger 
grains for the $\mathrm{x}=0.05$ system differ in shape from the elongated layered grains found in the $\mathrm{x}=0.01$ ceramic system.
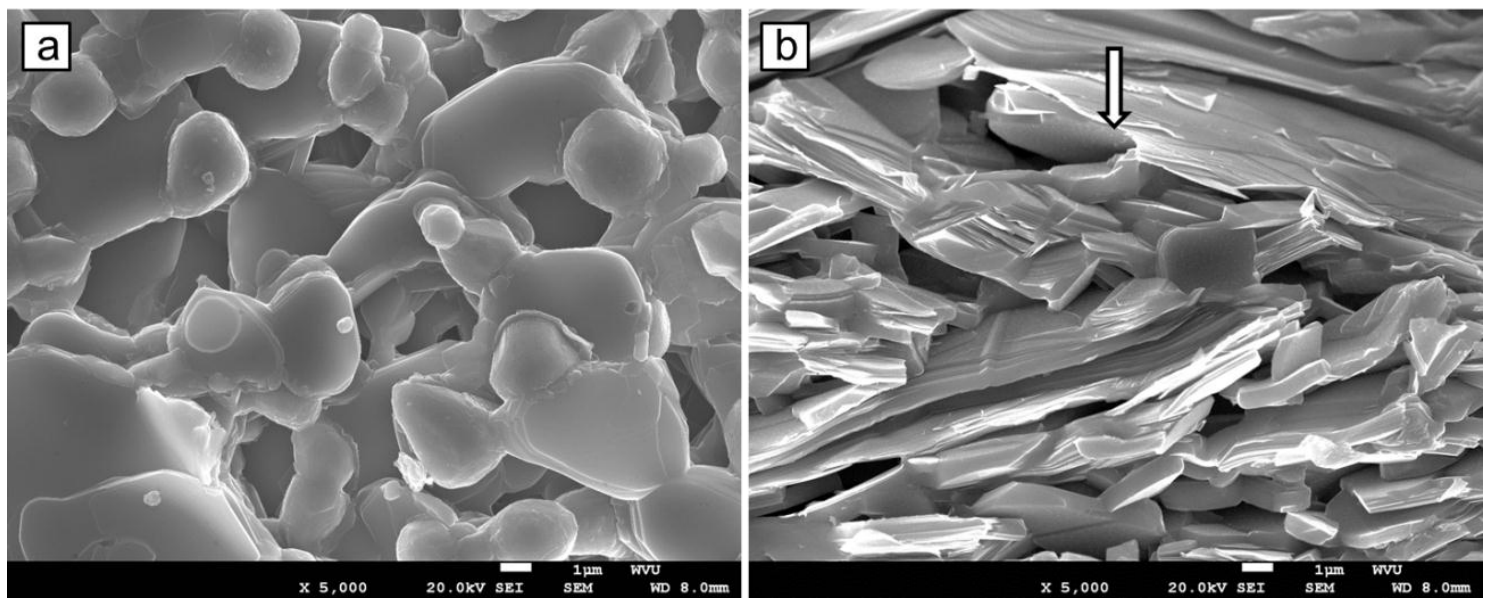

Figure 35. SEM micrographs of the $x=0.01$ ceramic system showing two surfaces: (a) the pressed plane and (b) a fractured cross-section. The arrow indicates the applied stress direction.
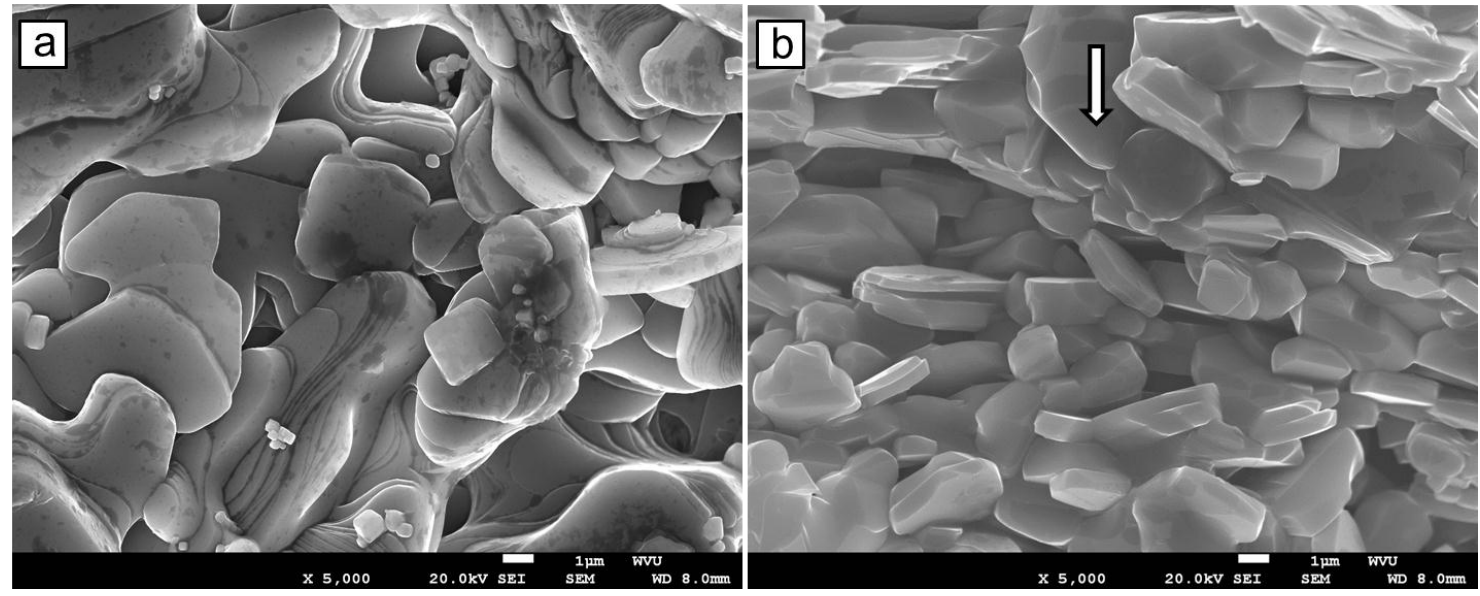

Figure 36. SEM micrographs of the $\mathrm{x}=0.05$ ceramic system showing two surfaces: (a) the pressed plane and (b) a fractured cross-section. The arrow indicates the applied stress direction.

The nanostructures of the $\mathrm{x}=0.01$ and 0.05 ceramic systems were inspected using TEM. Bright field micrographs of the specimens show, not only the typical polycrystalline structure of the ceramic, but the existence nanoparticles embedded within the ceramic grains as can be 
observed in Figure 37 and Figure 38 for the $x=0.01$ and 0.05 systems respectively. The imaged nanoparticles in the $\mathrm{x}=0.01$ ceramic present diameters ranging from 100 to $500 \mathrm{~nm}$. The nanoparticles of the $\mathrm{x}=0.05$ ceramic are larger, with lengths over $200 \mathrm{~nm}$.
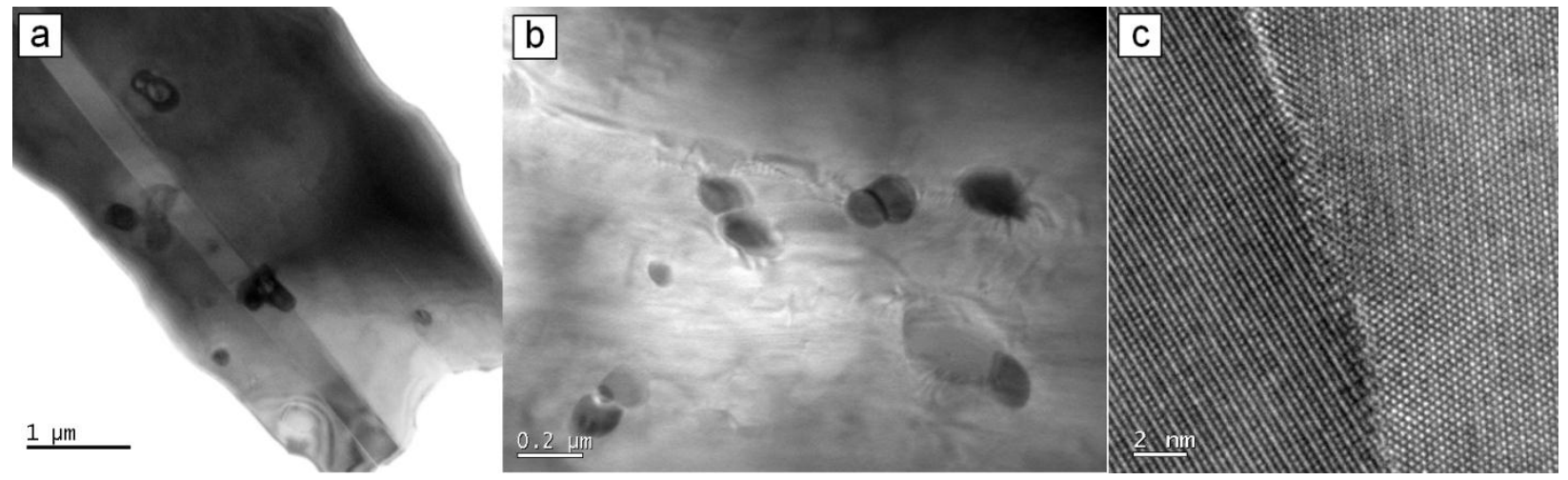

Figure 37. TEM bright field images (a) and (b) of nanoinclusions within ceramic grains of the $\mathrm{x}=0.01$ system. (c) HRTEM image of the grain boundary between a nanoparticle (left) and the CCO substrate (right).

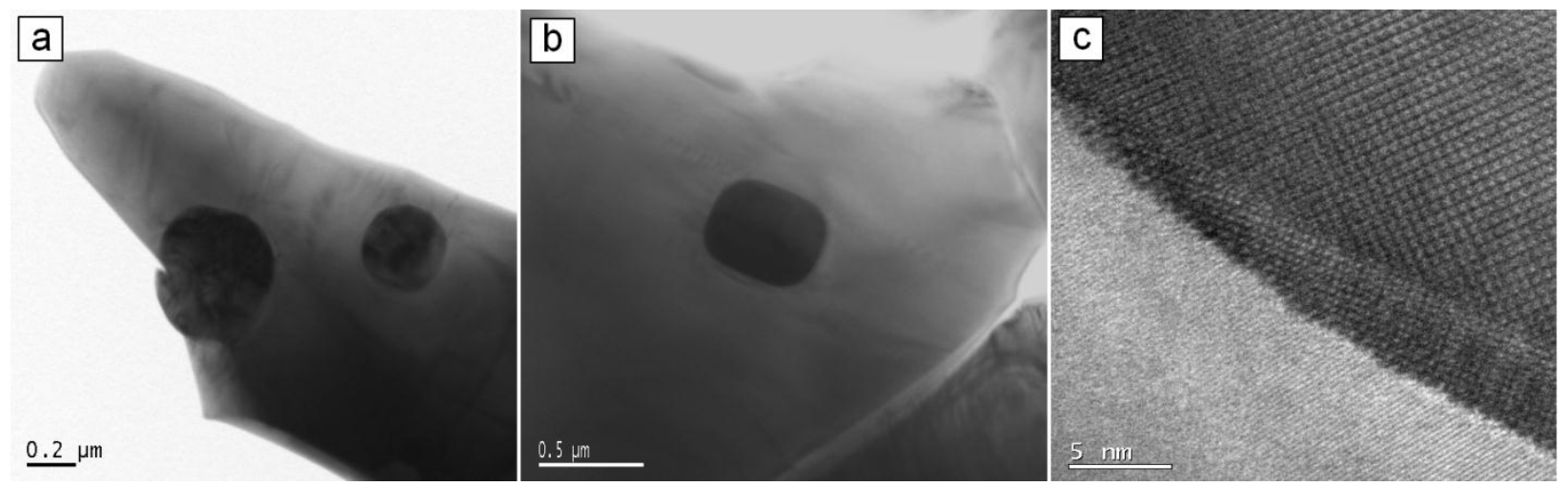

Figure 38. TEM bright field images (a) and (b) of the nanoinclusions within the ceramic grains of the $\mathrm{x}=0.05$ system. (c) HRTEM image of the grain boundary between a nanoparticle (right) and the CCO substrate (left).

EDS chemical analysis of the $\mathrm{x}=0.01$ and 0.05 specimens revealed that the ceramic bulks present $\mathrm{Ca}_{3} \mathrm{Co}_{4} \mathrm{O}_{9}$ nominal composition ( $\mathrm{Ca}$ to $\mathrm{Co}$ atomic ratio of 3:4) with no registration of $\mathrm{Pd}$ 
signal. The nanoparticles, in the other hand, presented high Pd content. Representative EDS data from the ceramic grains and nanoparticles is reported in Table 9.

Table 9. Representative EDS data from the ceramic grains and nanoparticles.

\begin{tabular}{|c|c|c|c|c|c|}
\hline \multirow{2}{*}{$\mathrm{x}$} & & \multicolumn{4}{|c|}{ atomic \% } \\
\hline & & $\mathrm{O}$ & $\mathrm{Ca}$ & Co & $\mathrm{Pd}$ \\
\hline \multirow{2}{*}{0.01} & ceramic grain & 40.12 & 26.12 & 33.76 & - \\
\hline & nanoparticle & 47.15 & 11.86 & 6.05 & 34.94 \\
\hline \multirow{2}{*}{0.05} & ceramic grain & 46.49 & 22.52 & 30.99 & - \\
\hline & nanoparticle & 41.62 & $\mid 12.32$ & 6.62 & 39.44 \\
\hline
\end{tabular}

Electron diffraction patterns of the nanoparticles were obtained in order to precisely determine the composition and crystal structure of the nanoparticles. Indexing of the diffraction patterns (along with the EDS data) allowed identifying the nanoparticles as the crystalline $\mathrm{Pd}_{3.5} \mathrm{O}_{4}$ cubic phase. Figure 39 (a) shows a TEM micrograph from the $\mathrm{x}=0.01$ system where a layered CCO grain containing a nanoinclusion can be appreciated. The electron diffraction in (b) corresponds to the $\mathrm{CCO}$ substrate while the one in (c) corresponds to the nanoparticle. The $c$-axis direction of the $\mathrm{CCO}$ grain can be determined from the diffraction pattern. The diffraction pattern in Figure 39 (c)Figure 39 was indexed as the [010] zone axis of the cubic $\mathrm{Pd}_{3.5} \mathrm{O}_{4}$ phase. Figure 40 details one of the nanoparticles embedded within the $\mathrm{CCO}$, it can be appreciated the structural coherency existing between the $\mathrm{CCO}$ substrate and the $\mathrm{Pd}_{3.5} \mathrm{O}_{4}$ nanoinclusion. 

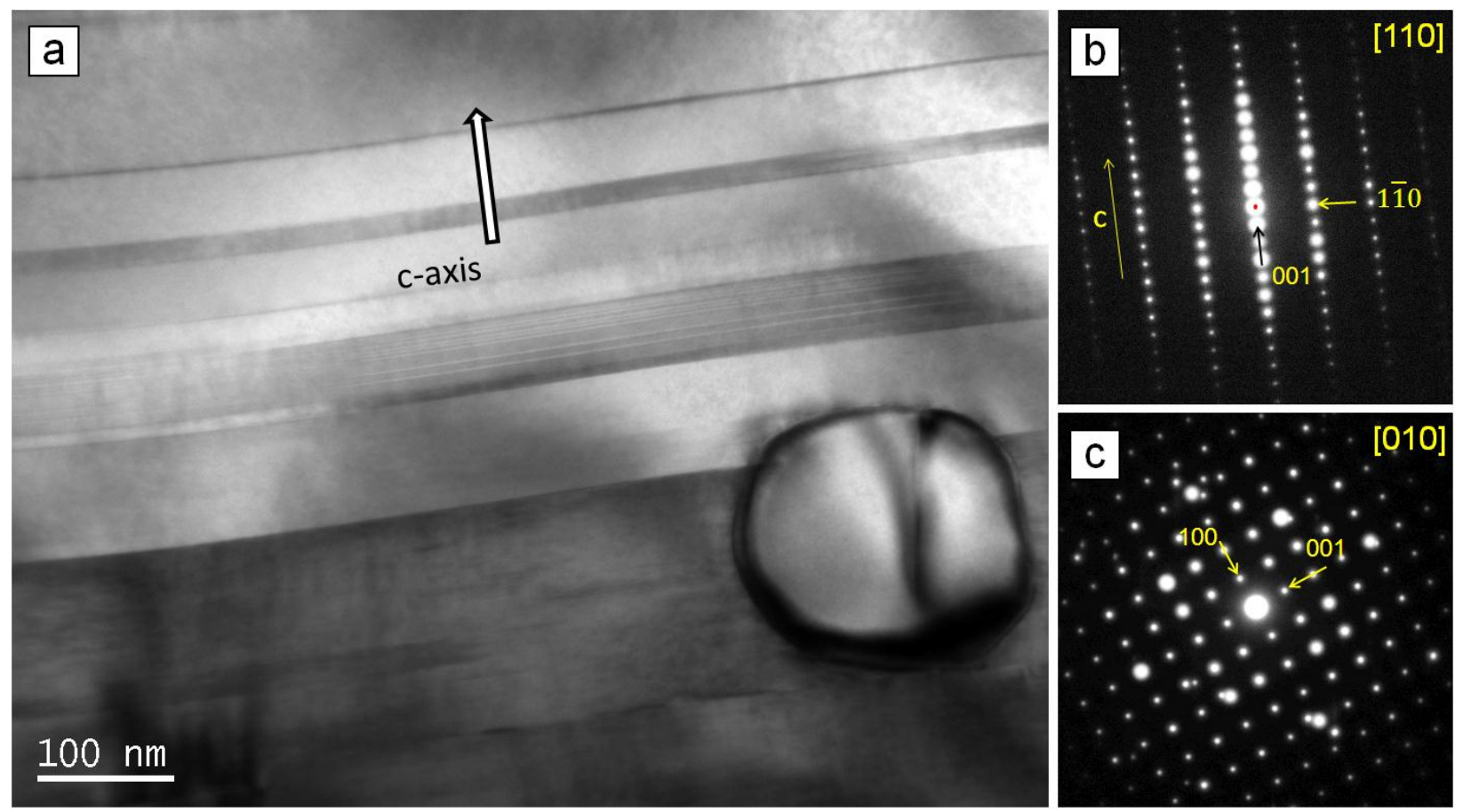

Figure 39. (a) TEM micrograph from the $\mathrm{x}=0.01$ system showing a $\mathrm{Pd}_{3.5} \mathrm{O}_{4}$ nanoparticle on the $\mathrm{CCO}$, (b) and (c) show the electron diffraction patterns from the $\mathrm{CCO}$ substrate and the $\mathrm{Pd}_{3.5} \mathrm{O}_{4}$ nanoinclusion respectively.

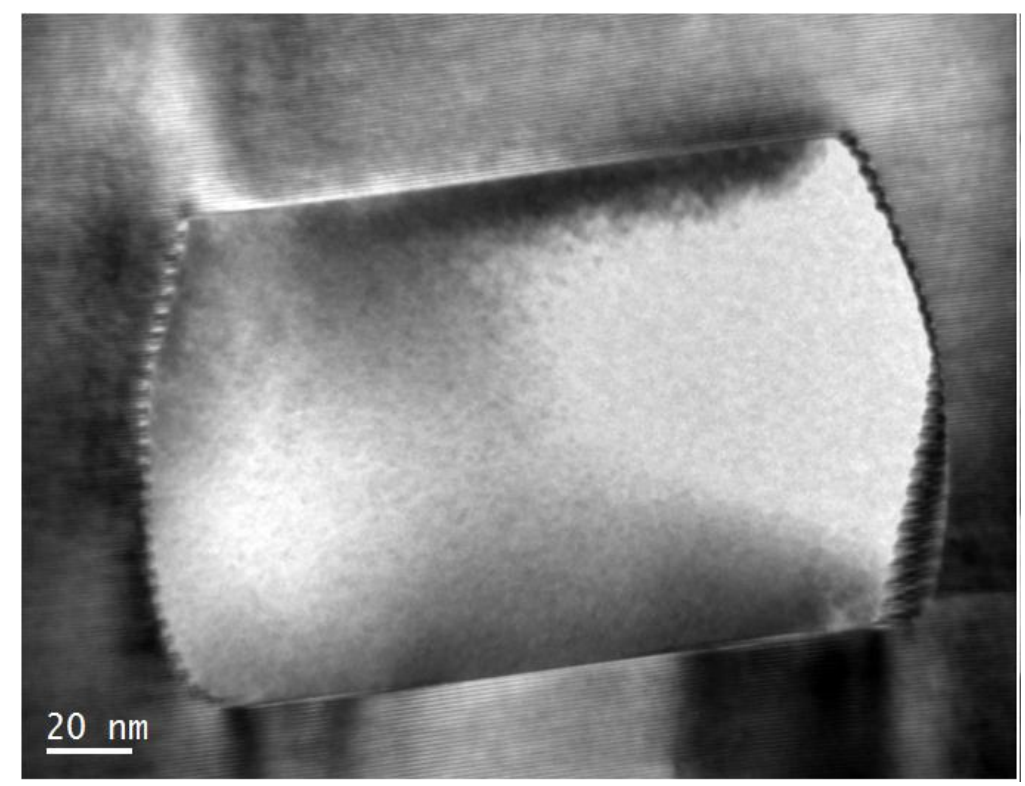

Figure 40. TEM micrograph showing a $\mathrm{Pd}_{3.5} \mathrm{O}_{4}$ nanoinclusion embedded within the $\mathrm{CCO}$ grain the $\mathrm{x}=0.01$ ceramic system. 
Figure 41 shows two electron diffraction patterns from two different zone axes of one of the nanoparticles observed in the $\mathrm{x}=0.05$ composite. As for the previous case, the diffraction patterns correspond to the crystalline $\mathrm{Pd}_{3.5} \mathrm{O}_{4}$ cubic phase.

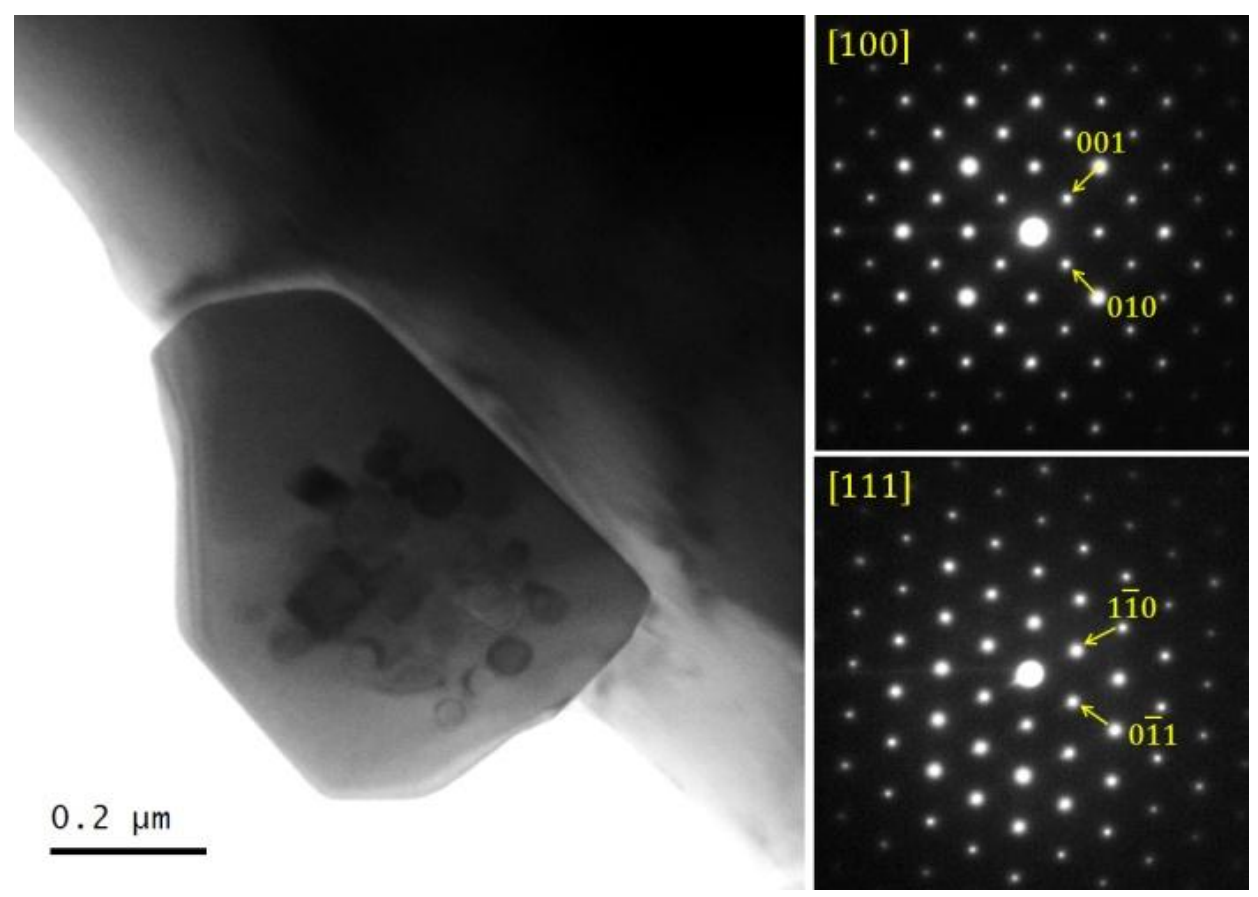

Figure 41. TEM micrograph and two electron diffraction patterns from a nanoparticle of the $\mathrm{x}=0.05$ ceramic. The diffraction patterns were indexed as the [100] and [111] zone axes of the cubic $\mathrm{Pd}_{3.5} \mathrm{O}_{4}$ phase.

$S, \rho$ and power factor of the experimental $\mathrm{CCO} / \mathrm{Pd}$ ceramic systems $(\mathrm{x}=0,0.01$ and 0.05$)$ are presented in Figure 42. The $S$ - $T$ curves increase monotonically with the temperature and present p-type behavior. The $S$ - $T$ curves also show a significant reduction of $S$ for the Pd added samples especially at room temperature. For the $\mathrm{x}=0.01$ system the reduction at room temperature was $10.6 \%$ respect to the baseline and $12.0 \%$ for the $\mathrm{x}=0.05$ system. At $800^{\circ} \mathrm{C}$, however, the $S$ values are almost identical to the baseline. The resistivity of the CCO was notably reduced for the $\mathrm{x}=0.01$ sample while it was increased for the $\mathrm{x}=0.05$ system. As a result, the $\mathrm{x}=0.01$ sample 
presented the highest power factor while the $\mathrm{x}=0.05$ sample showed the poorest performance. The largest power factor improvement of $34 \%$ occurred at $730^{\circ} \mathrm{C}$ for the $\mathrm{x}=0.01$ system while the power factor reduction of the $\mathrm{x}=0.05$ sample was $30.8 \%$ in average for the entire measured temperature interval.
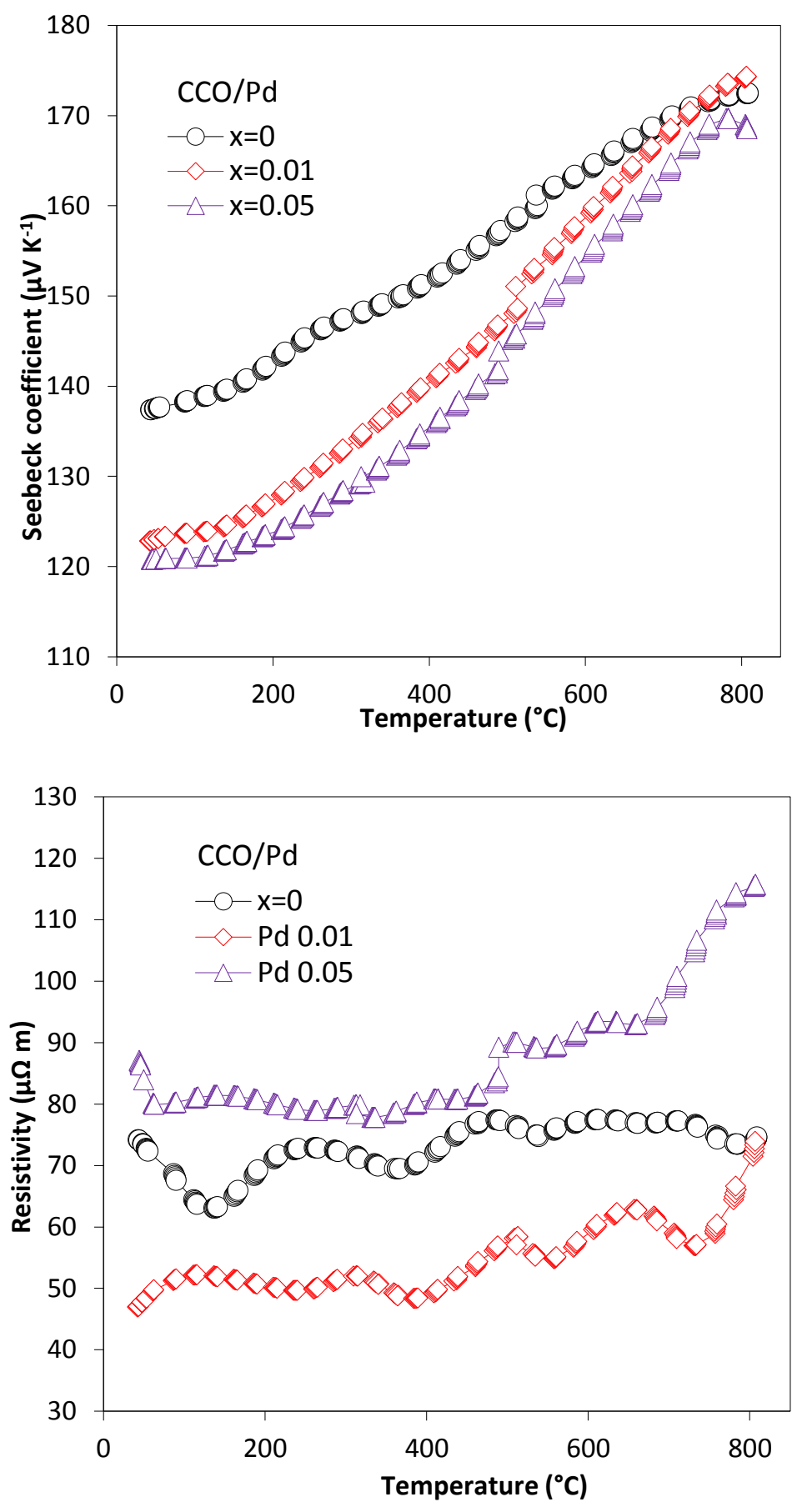


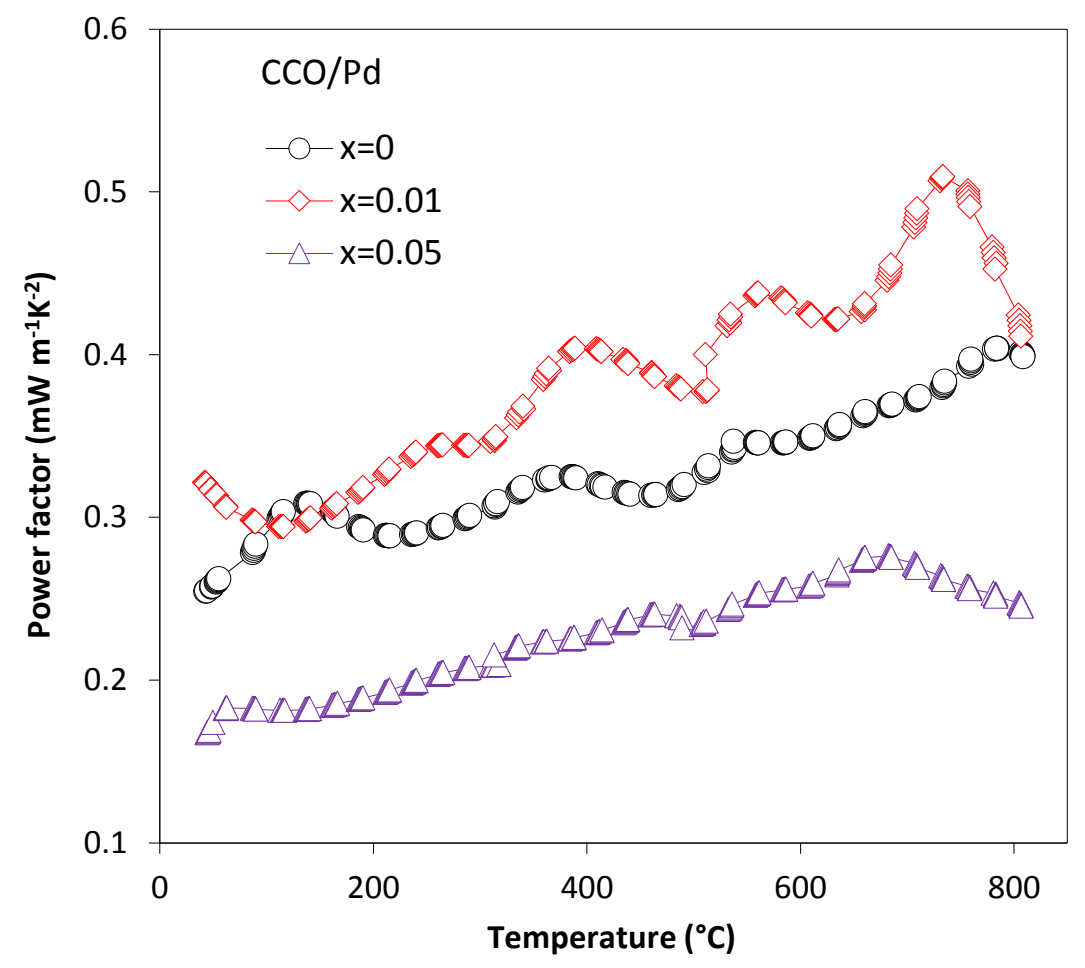

Figure 42. Seebeck coefficient, electrical resistivity and power factor of the experimental $\mathrm{CCO} / \mathrm{Pd}$ systems ( $\mathrm{x}=0,0.01$ and 0.05$)$.

\subsection{Effect of Au Addition on the Thermoelectric Properties of $\mathrm{Ca}_{3} \mathrm{Co}_{4} \mathrm{O}_{9}$ Ceramics}

\subsubsection{Experimental procedure}

The experimental $\mathrm{Ca}_{3} \mathrm{Co}_{4} \mathrm{O}_{9} / \mathrm{Au}-10 \mathrm{wt} \%$ composite ceramic was produced by combining the sol-gel method with hydrothermal deposition. The first step was the synthesis of the CCO powder by sol-gel route; the solution preparation process for the $\mathrm{CCO}$ powder was the same used previously for the synthesis of the pure CCO powder using 50\% EG as solvent. After CCO powder synthesis, Au nanoparticles were added by using hydrothermal deposition. This 
procedure differs significantly from the other ones employed previously in this research for the formation of nanoparticles, where the precursors were directly dissolved into the sol-gel.

The hydrothermal deposition process was performed following the deposition-precipitation with urea method (DP Urea) reported by Zanella et al. that allows obtaining highly dispersed gold nanoparticles (2-3 nm) for high Au loads [47]. The precursor solution was prepared by dissolving and mixing the synthesized $\mathrm{CCO}$ powder with gold chloride $\mathrm{HAuCl}_{4} \cdot 3 \mathrm{H} 20$ and urea $\mathrm{Co}\left(\mathrm{NH}_{2}\right)_{2}$ in deionized water. The concentration of gold chloride was set to obtain a $10 \mathrm{wt} \% \mathrm{Au}$ load and the urea concentration was used to be 100 times that of gold chloride. The role of the urea was to serve as precipitating base, producing a gradual and homogeneous addition of hydroxide ions in the solution. After dissolution, the solution was placed into the hydrothermal reactor where the thermal cycle illustrated in Figure 43 was implemented. A temperature of $90^{\circ} \mathrm{C}$ was applied for a dwell time of $10 \mathrm{~h}$ while stirring the solution at $1000 \mathrm{rpm}$. Reaction of the reagents occurred during this process to produce the deposition of Au nanoparticles over the CCO powder grains. The resultant powder was washed and centrifuged several times. Finally, based in the procedure reported by Delannoy et al., the collected powder was calcined in air at $300^{\circ} \mathrm{C}$ for $4 \mathrm{~h}$ in order to remove any organic residue [48].

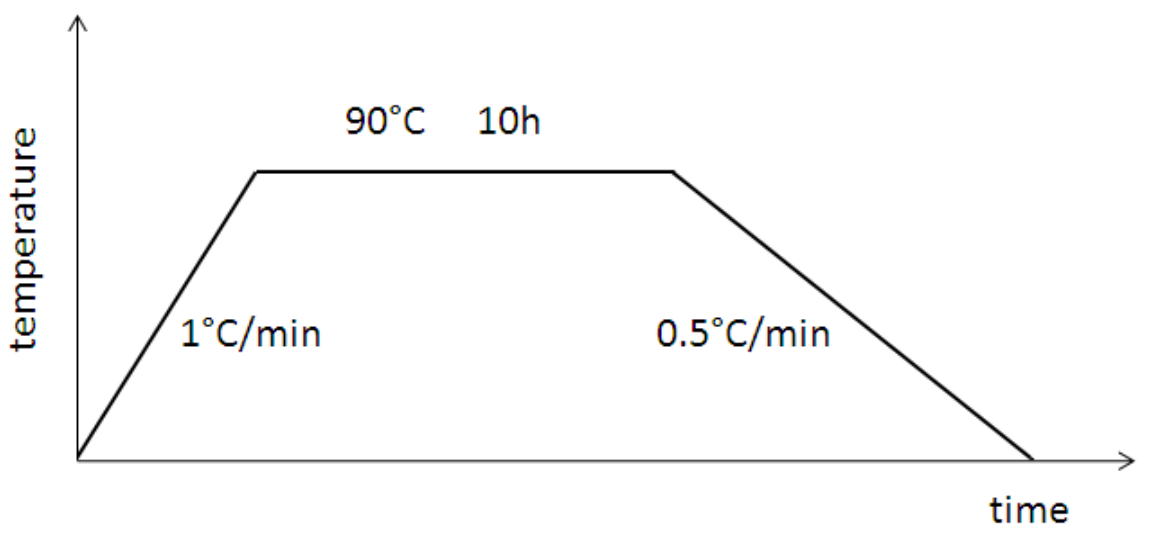

Figure 43. Temperature profile as a function of time applied during hydrothermal Au deposition. 
As mention before, the hydrothermal $\mathrm{Au}$ deposition was performed after calcination of the CCO powder. The resultant power was subsequently consolidated into pellets by hot-pressing under $1 \mathrm{GPa}$ pressure at $150^{\circ} \mathrm{C}$ during 30 minutes followed by sintering in a box furnace during 18 hours at $920^{\circ} \mathrm{C}$. A flow diagram of the entire preparation procedure is presented in Figure 44. An additional pure CCO sample (without $\mathrm{Au}$ addition) was prepared under the same conditions to serve as baseline for the $S, \rho$ and power factor measurements.

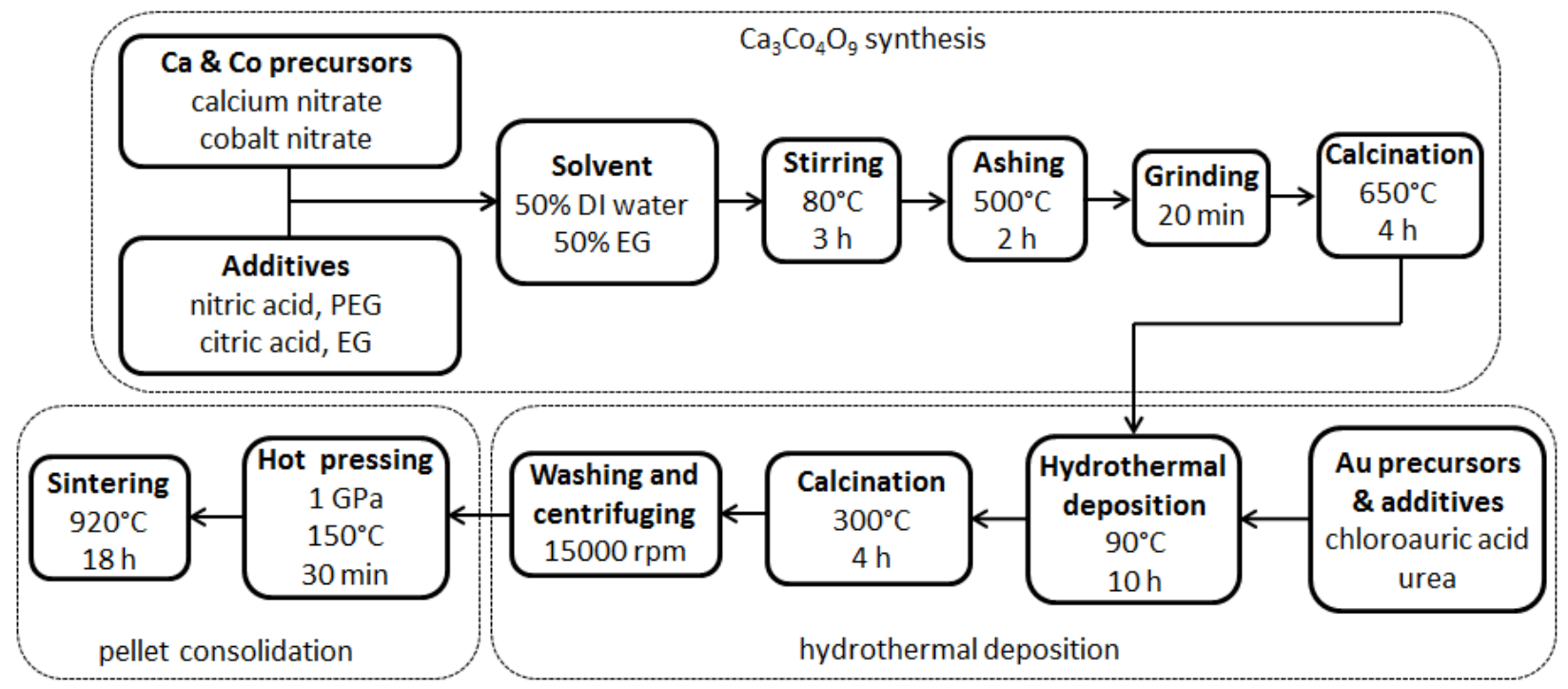

Figure 44. Flow diagram of the fabrication route of the CCO/Au-10 wt $\%$ composite ceramic.

TEM analysis of the powder after hydrothermal deposition of Au was performed using a JEOL TEM-2100 operating at $200 \mathrm{kV}$ equipped with EDS analyzer. TEM samples were prepared by dispersing the powder in alcohol followed by deposition in a carbon film supported by a copper grid. Pressed plane and cross-sectional fractured surface of the consolidated $\mathrm{CCO} / \mathrm{Au}$ ceramic were imaged by SEM using a JEOL JSM-7600F microscope. TEM-EDS analysis of the consolidated ceramic was also performed to specimens prepared by conventional polishing of the samples using abrasive films. Indexing of the electron diffraction patterns was assisted by the ICDD PDF-4+ version 4.1203 software. $S, \rho$ and power factor measurements were performed in 
the direction parallel to the pressed plane from room temperature up to $800^{\circ} \mathrm{C}$ using a Linseis LSR-1100 equipment in a He environment.

\subsubsection{Results and discussion}

TEM analysis of the synthesized $\mathrm{CCO} / \mathrm{Au}$ powder was performed after the hydrothermal deposition of $\mathrm{Au}$ on the CCO. Figure 45 presents the morphology of the synthesized powder at the nano-scale. It can be observed how CCO powder serves as support for diminutive spherical Au nanoparticles with average size of $4 \mathrm{~nm}$ and narrow size distribution. The occurrence of the nanoparticles is homogeneous throughout the $\mathrm{CCO}$ powder.
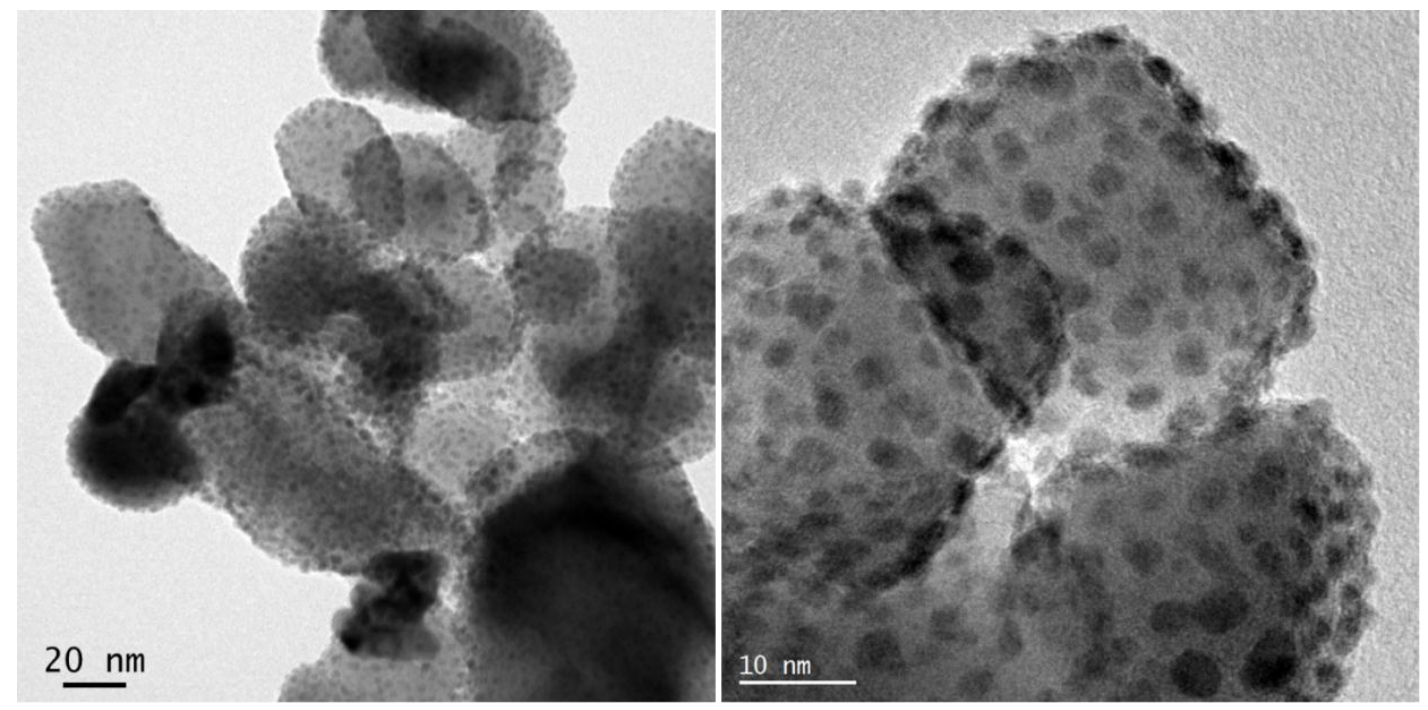

Figure 45. TEM micrograph of the hydrothermally synthesized $\mathrm{CCO} / \mathrm{Au}$ powder.

SEM analysis of the consolidated pellet revealed that the CCO/Au ceramic presents spherical Au nanoparticles uniformly distributed all over the CCO grains as can be observed in Figure 46 and Figure 47. The nanoparticles are much larger than those observed in the powder after hydrothermal deposition (Figure 45), meaning that growth of the Au nanoparticles occurred during the hot-pressing and sintering procedures executed afterwards. The imaged nanoparticles 
present spherical/faceted shapes. Regardless of the nanoparticles, the ceramic morphology is similar to those obtained previously in this study; characteristics such as high porosity and poor alignment can be appreciated.

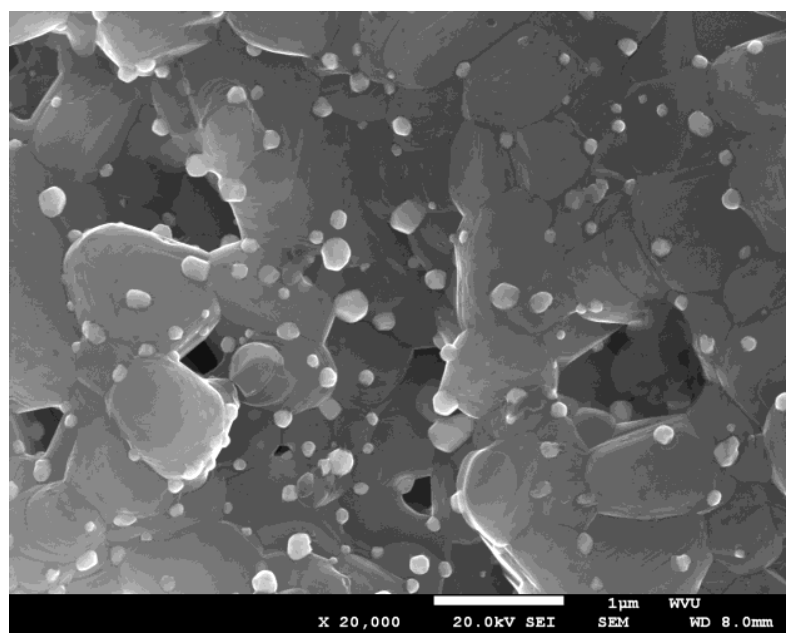

Figure 46. SEM micrograph of the prepared CCO/Au composite ceramic showing Au nanoparticles uniformly distributed all over the CCO.
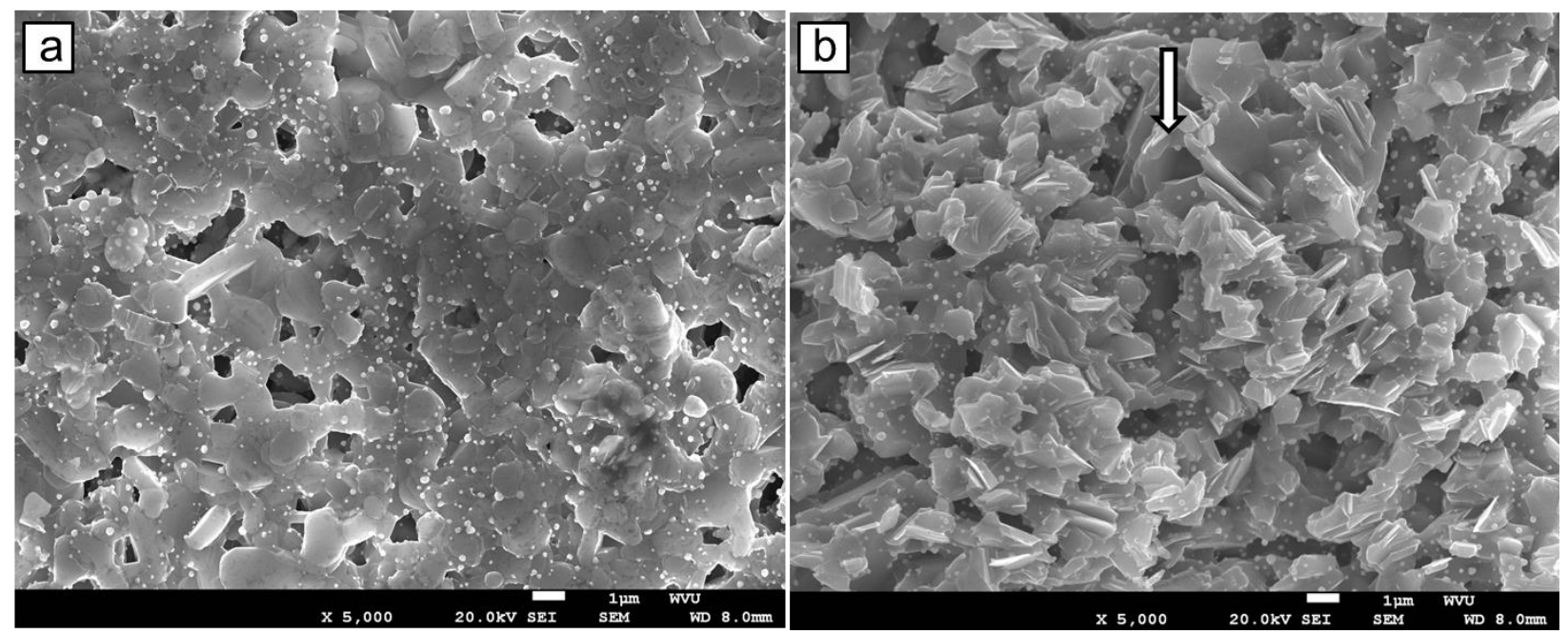

Figure 47. SEM micrographs from: (a) the pressed plane and (b) the fractured cross-section of the $\mathrm{CCO} / \mathrm{Au}$ pellet. The arrow indicates the direction of the applied pressing. 
TEM micrographs from the consolidated $\mathrm{CCO} / \mathrm{Au}$ ceramic system are presented in Figure 48. Au nanoparticles can be observed to be homogeneously distributed all over the CCO grains. It can also be appreciated Au nanoparticles with smaller size than those observed previously in the SEM micrographs (Figure 46), this means that not all the hydrothermally deposited Au grew during the ceramic sintering. The smaller nanoparticles are observed to be located within the layered CCO grains or at the grain boundaries. The size of the nanoparticles can be explained from their location in the ceramic; the $\mathrm{Au}$ nanoparticles embedded within the $\mathrm{CCO}$ grains (nanoinclusions) or confined at the grain boundaries are dimensionally constrained leading to low growth during sintering. The nanoparticles at the CCO surface, instead, have no spatial restrictions and can grow more easily.

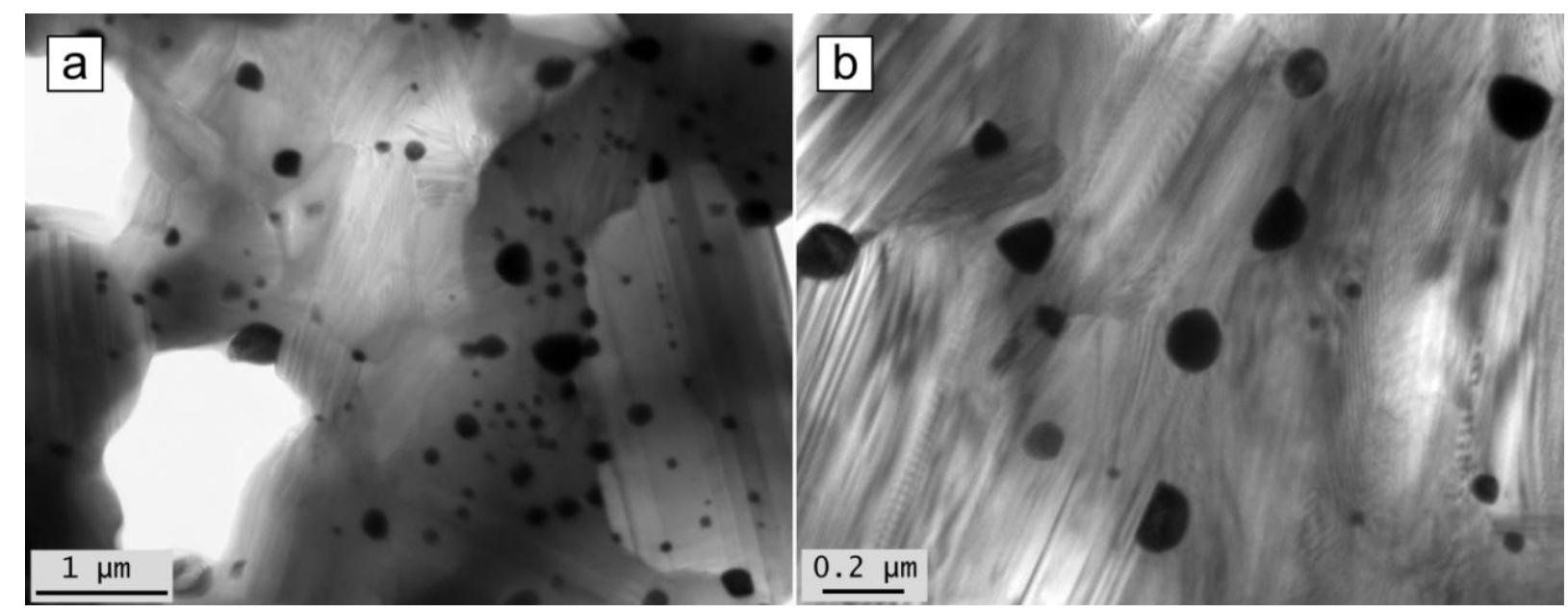

Figure 48. TEM micrographs from the nanostructure of the $\mathrm{CCO} / \mathrm{Au}$ ceramic system. $\mathrm{Au}$ nanoparticles can be observed all over the $\mathrm{CCO}$ grains.

Figure 49 (a) shows a TEM micrograph of an Au nanoinclusion embedded within the layered nanostructure of the $\mathrm{CCO}$; it can be observed the structural coherency existing between the nanoinclusion and the substrate. From the EDS analysis of the CCO substrate it was determined that no doping of the $\mathrm{CCO}$ ceramic occurred (no Au signal was registered) meaning that all the 
added Au contributed to the formation of nanoparticles. The electron diffraction pattern in Figure 49 (b) corresponds to the CCO substrate and matches the [210] zone axis of the monoclinic CCO structure. The one in (c) corresponds to the Au nanoparticle and was indexed as the [211] zone axis of the cubic Au phase.

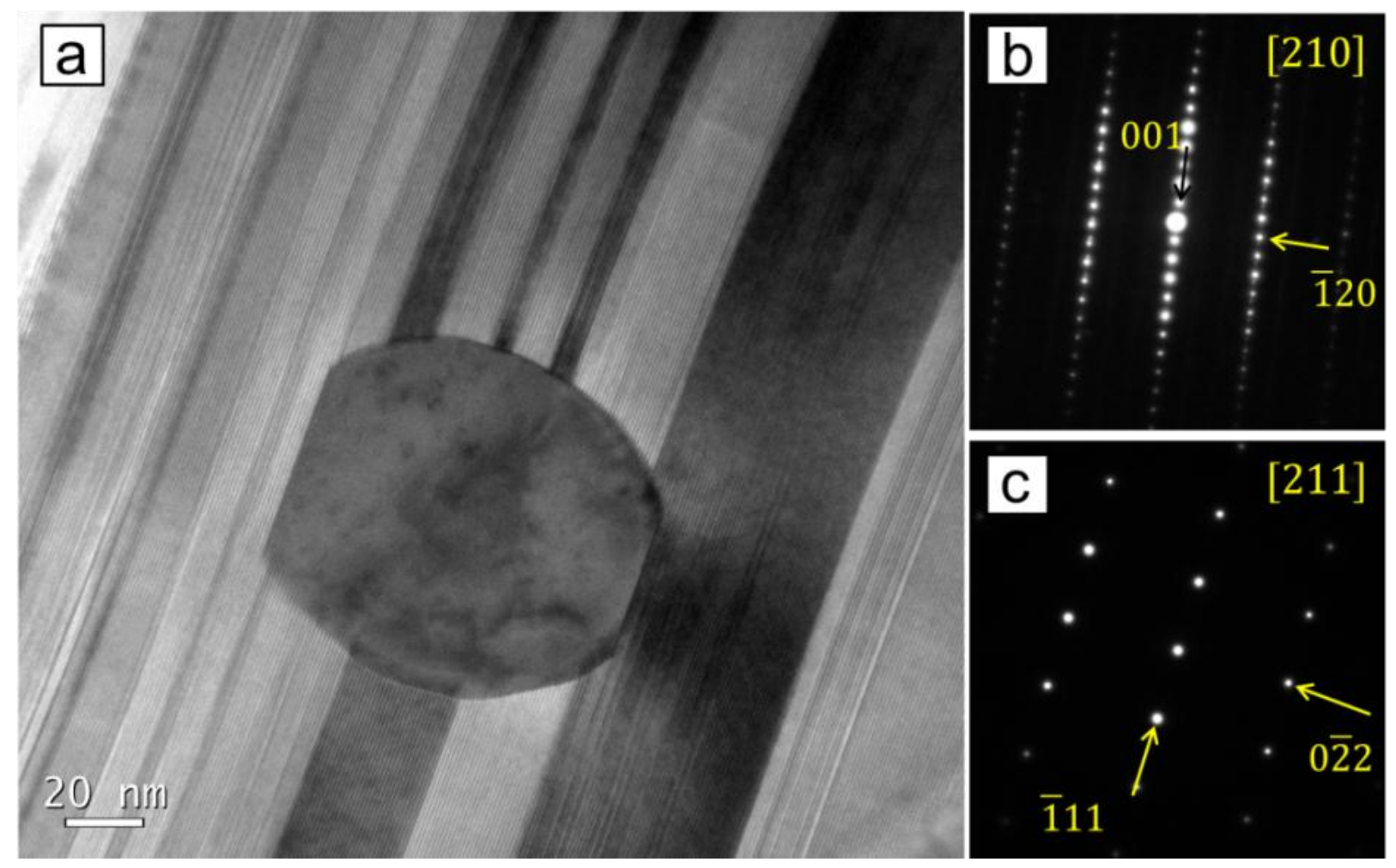

Figure 49. (a) TEM micrograph of an Au nanoinclusion embedded within the layered nanostructure of the $\mathrm{CCO}$, (b) and (c) electron diffraction patterns of the $\mathrm{CCO}$ substrate and the Au nanoparticle respectively.

Figure 50 exhibits two different locations of the Au nanoparticles that are expected to produce an improvement of the TE performance: within the $\mathrm{CCO}$ grains (a) and at the grain boundaries (b). 


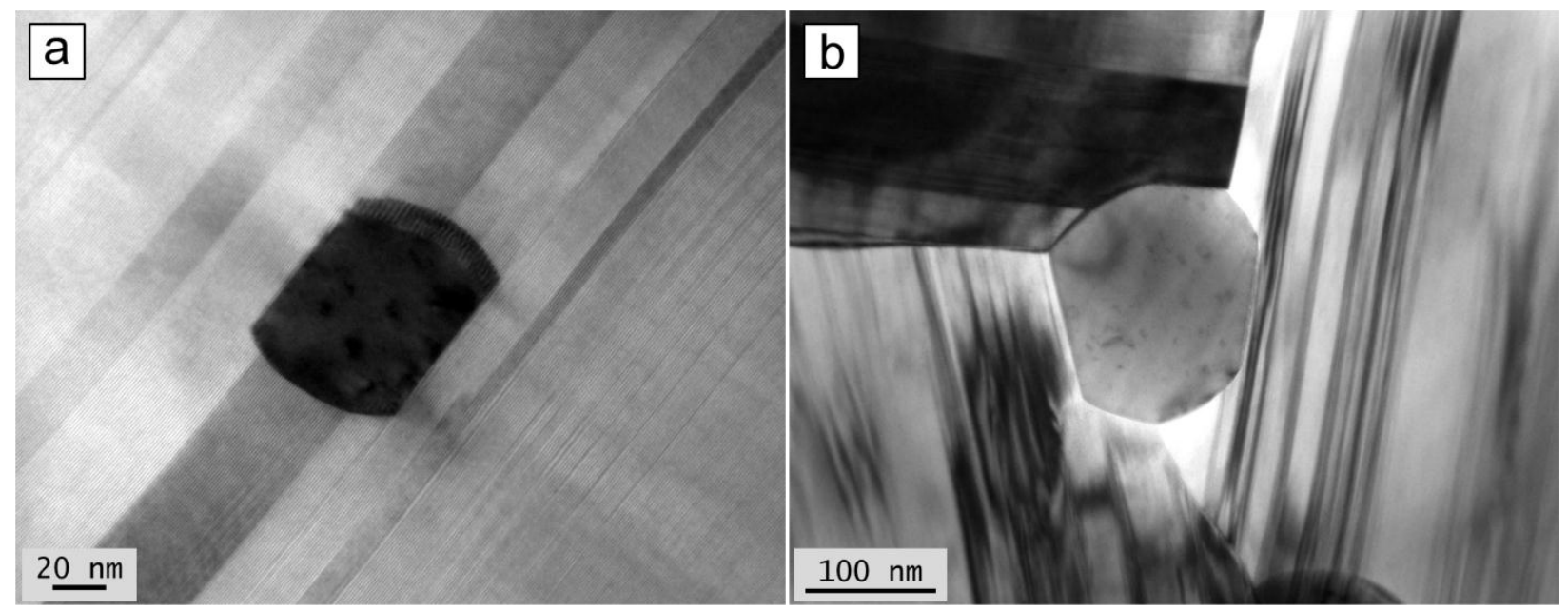

Figure 50. Bright field TEM micrographs of Au nanoparticles located (a) within the CCO and (b) at the grain boundary.

$S, \rho$ and power factor of the prepared $\mathrm{CCO} / \mathrm{Au}$ composite are presented in Figure 51 along with the baseline data. As can be observed, $S$ was substantially reduced for the entire temperature range due to the addition of $\mathrm{Au}$; the reduction was of $10.0 \%$ in average and $13.8 \%$ at $800^{\circ} \mathrm{C}$. Electrical conductivity was also reduced by $\mathrm{Au}$ alloying, especially for temperatures above $400^{\circ} \mathrm{C}$ where the curves branch out. The variation rate of $\rho$ respect to the temperature was notably altered as shown in the graph; the resistivity magnitude goes from $8.3 \%$ above the baseline at $400^{\circ} \mathrm{C}$ to $40.4 \%$ at $800^{\circ} \mathrm{C}$. Consequently, the power factor resulted affected for the variations of both $S$ and $\rho$ as can be observed in the respective graph. An average magnitude reduction of $31.2 \%$ was obtained for the entire temperature range respect to the baseline; at $800^{\circ} \mathrm{C}$ the difference was of $47 \%$ below. From this data, it is evident that thermoelectric performance of CCO was deteriorated by the action of the deposited Au nanoparticles. Neither $S$ nor $\rho$ was favored by the action of Au alloying, contributing both to reduce the power factor. 

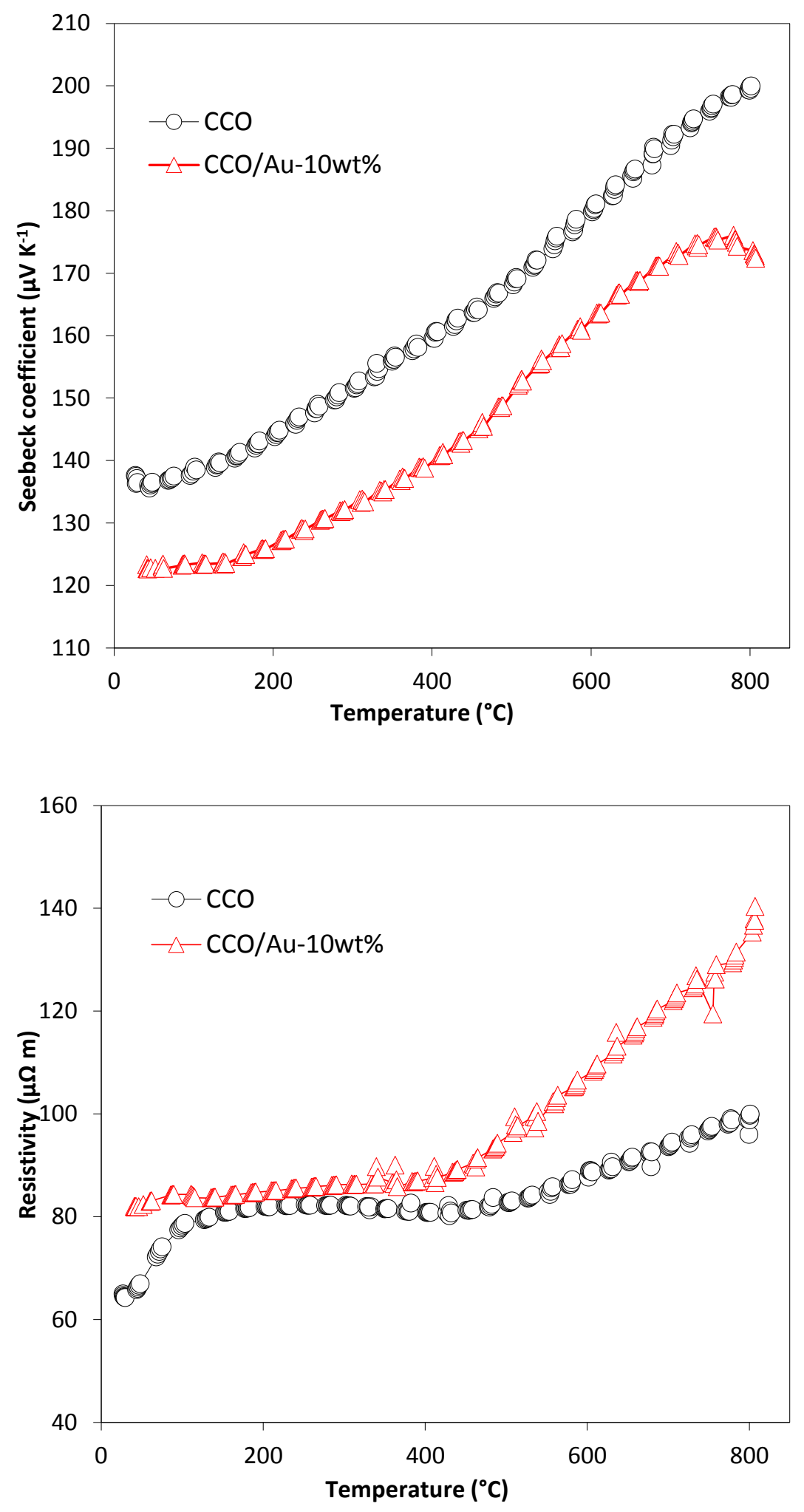


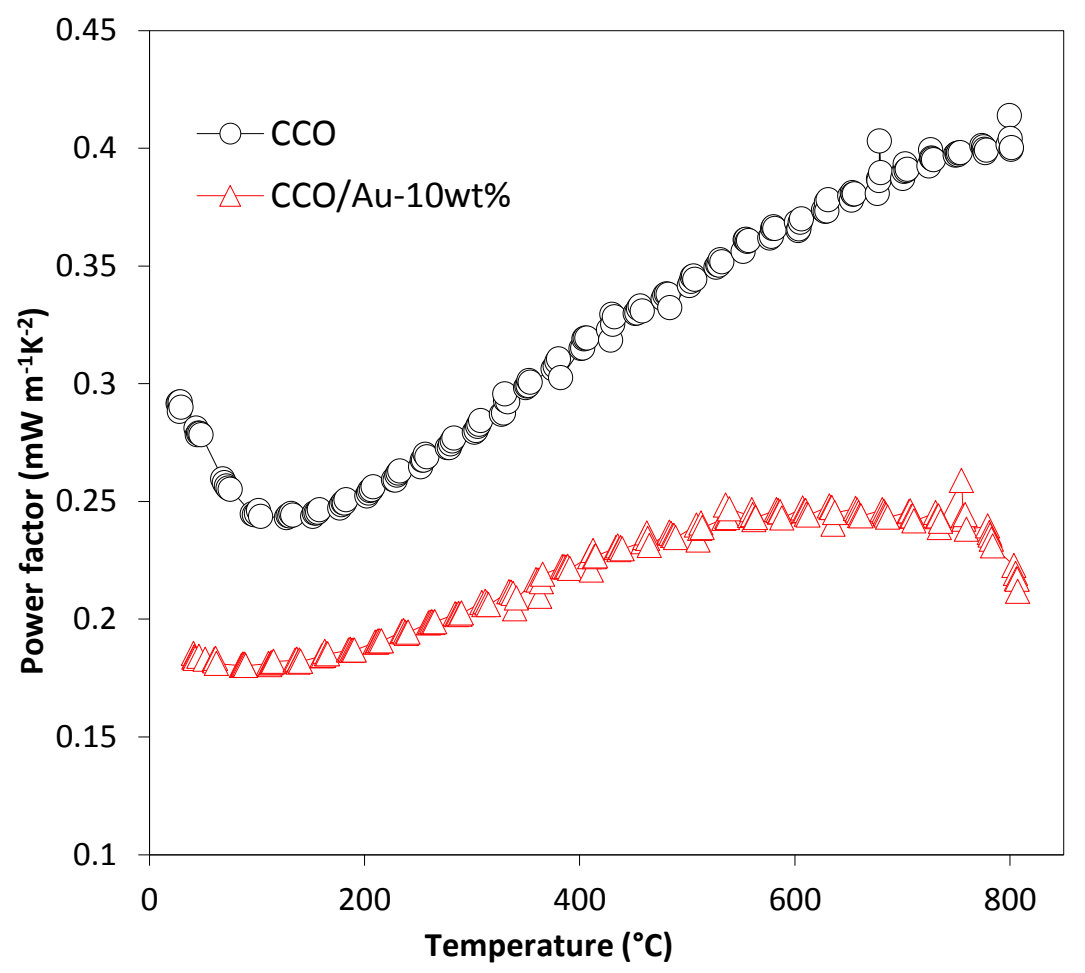

Figure 51. Seebeck coefficient, resistivity and power factor of the CCO/Au-10 wt\% system compared with the baseline data. 


\section{CHAPTER 6: CONCLUSIONS AND FUTURE WORK}

Differences in the thermoelectric performance of the CCO samples prepared using different solvents (Chapter 3) were observed. From to behavioral changes registered, the resistivity appears to be particularly sensitive to the type of solvent. The CCO sample prepared using 50\% EG as solvent showed the largest $S$ values at high temperature and also the largest power factor for the measured temperature range. The sample prepared using deionized water showed the lowest power factor while the DEG sample showed intermediated values. The experimental data obtained from the microstructure and nanostructure of the samples, however, resulted insufficient to explain the changes in the thermoelectric performance since the different samples present similar characteristics in terms of chemical composition, alignment, porosity and layered structure. This experiment, therefore, needs to be adjusted in order to obtain more conclusive results.

The addition of YSZ (8 mole \%) to the CCO for the formation of the CCO/YSZ-2 wt\% system resulted in doping of the CCO by Zr. Not all the Zr from the YSZ, however, doped the $\mathrm{CCO}$; large clusters formed by nonstoichiometric CCO and YSZ were found throughout the ceramic. According to the thermoelectric measurements performed it can be stated that the addition of YSZ does not affect the power factor of the CCO under the synthesis and consolidation methods employed.

Several outcomes resulted in the chemistry and microstructure of the CCO from the addition of hydrothermal $\mathrm{CoAl}_{2} \mathrm{O}_{4}$ nanopowder for the preparation of the $\mathrm{CCO} / \mathrm{CoAl}{ }_{2} \mathrm{O}_{4}-10 \mathrm{wt} \%$ ceramic alloy. The $\mathrm{CoAl}_{2} \mathrm{O}_{4}$ phase completely dissociated during the synthesis-consolidation process of the ceramic composite since no evidence of this crystal system was found in the consolidated ceramic. Aluminum ions from the added $\mathrm{CoAl}_{2} \mathrm{O}_{4}$ were found in two conditions: doping the 
$\mathrm{CCO}$ and combined with oxygen in an amorphous phase distributed all through the ceramic. The cobalt ions, on the other hand, produced the $\mathrm{Co}_{3} \mathrm{O}_{4}$ phase that was found in the form of irregular grains distributed along with the $\mathrm{Al}$ doped $\mathrm{CCO}$ grains. The addition of the $\mathrm{CoAl}_{2} \mathrm{O}_{4}$ deteriorated the power factor of the $\mathrm{CCO}$, especially at high temperature where a reduction close to $50 \%$ occurred. The Seebeck coefficient was reduced at low temperature while the resistivity severely increased at high temperature.

For the case of the $\mathrm{CCO} / \mathrm{NiFe}_{2} \mathrm{O}_{4}-2 \mathrm{wt} \%$ composite ceramic; the addition of $\mathrm{NiFe}_{2} \mathrm{O}_{4}$ nanopowder resulted in dissociation of the $\mathrm{NiFe}_{2} \mathrm{O}_{4}$ phase during the synthesis-consolidation process of the ceramic alloy. Fe ions doped the CCO structure while the Ni ions formed two different cobalt nickel oxide crystal phases: $\mathrm{Co}_{0.5} \mathrm{Ni}_{0.5} \mathrm{O}$ and $\mathrm{Co}_{2} \mathrm{NiO}_{4}$. These phases can be observed distributed throughout the ceramic microstructure with size ranging from 0.1 to $1 \mu \mathrm{m}$. The $\mathrm{Co}_{3} \mathrm{O}_{4}$ crystal phase was also observed in this ceramic in the form of irregular grains coexisting along with the Fe-doped CCO grains. Although the Seebeck coefficient was not affected by the $\mathrm{NiFe}_{2} \mathrm{O}_{4}$ addition, the conductivity was severely deteriorated: the resistivity increased $79 \%$ (in average) respect to the resistivity of the pure CCO.

From the addition of Pd metal into the $\mathrm{CCO}$ ceramic during the sol-gel synthesis process, it was expected to produce the $\mathrm{Ca}_{3} \mathrm{Co}_{4-\mathrm{x}} \mathrm{Pd}_{\mathrm{x}} \mathrm{O}_{9}(\mathrm{x}=0.01,0.05)$ systems by doping the $\mathrm{Pd}$ ions into the CCO. Results, however, revealed that no Pd ions entered the CCO lattice for any of the two concentrations tested; meaning that no chemistry modification of the CCO system occurred. The $\mathrm{Pd}$ instead, reacted with oxygen to produce the cubic $\mathrm{Pd}_{3.5} \mathrm{O}_{4}$ crystal system leading to the formation of nanoinclusions distributed all through the ceramic composite. The nanoparticles observed in the two prepared $\mathrm{CCO} / \mathrm{Pd}$ systems present different sizes, being larger for the $\mathrm{x}=0.05$ Pd concentration. The Seebeck coefficient was notably deteriorated towards room temperature 
by the addition of Pd for the two different concentrations. The, resistivity on the other hand, was reduced for the $\mathrm{x}=0.01 \mathrm{Pd}$ concentration and increased for the $\mathrm{x}=0.05$ one. As a consequence, an improvement of the power factor was obtained for the $\mathrm{x}=0.01 \mathrm{Pd}$ concentration while it was deteriorated for the $\mathrm{x}=0.05$ case.

The addition of $\mathrm{Au}$ into the $\mathrm{CCO}$ ceramic powder by hydrothermal deposition produced a large quantity of $\mathrm{Au}$ nanoparticles homogeneously distributed throughout the $\mathrm{CCO}$ ceramic; nanoparticles were found within the $\mathrm{CCO}$ grains, at the grain boundaries and grain surfaces. Particles present a wide size distribution (50 to $500 \mathrm{~nm}$ ), with the larger grains located at the surfaces of the $\mathrm{CCO}$ grains and the smaller ones at the grain boundaries and within the CCO grains. No evidence of Au doping into the $\mathrm{CCO}$ was registered and no reaction phases between $\mathrm{Au}$ with the $\mathrm{CCO}$ or any of its constituent elements were found. Addition of Au drastically deteriorated the power factor of the $\mathrm{CCO}$ ceramic due to negative effects over both the Seebeck coefficient and the resistivity.

Although the power factor of the $\mathrm{CCO}$ got reduced for most of the performed experiments it doesn't mean that the addition of the different oxides and metals unavoidably deteriorate the thermoelectric performance of the $\mathrm{CCO}$. At this point no measurement of the thermal conductivity of the different ceramic systems has been performed so their figures-of-merit $Z$ (conversion efficiencies) are still unknown. It is possible the event of a substantial reduction of the thermal conductivity after alloying (especially for the systems with induced nanoinclusions as the $\mathrm{CCO} / \mathrm{Au}, \mathrm{CCO} / \mathrm{Pd}$ and $\mathrm{CCO} / \mathrm{NiFe}_{2} \mathrm{O}_{4}$ ) leading to the improvement of $Z$ despite the reduction of the power factor. In view of that, the next step of this research work is to determine the figure-of- merit of the tested systems by measurement of the thermal conductivities. 
Moreover, it could be tested the combined effect of doping with the incorporation of nanoinclusions based on the obtained results. For example, elements known to dope the CCO such as $\mathrm{Fe}$ and $\mathrm{Zr}$ could be used along with $\mathrm{Au}, \mathrm{Pd}$ or $\mathrm{Ni}$ in order to simultaneously dope the $\mathrm{CCO}$ and induce the formation of nanoinclusions which could result in $\mathrm{CCO}$ composites with superior conversion efficiencies.

Respect to the synthesis procedures, it was observed that the hydrothermal route is an effective technique to induce the formation of $\mathrm{Au}$ nanoinclusions within the $\mathrm{CCO}$ by hydrothermal deposition. The experimental conditions, however, need to be adjusted to avoid excessive growth of the Au nanoparticles. This deposition method could be also tested for the incorporation of other type of nanoparticles (for example $\mathrm{Pd}_{3.5} \mathrm{O}_{4}$ and $\mathrm{Co}_{2} \mathrm{NiO}_{4}$ ).

Finally, in terms of the fabrication of the $\mathrm{CCO}$ composites, it was observed that the alignment and density of the hot-pressed samples are still poor, possibly due to grain growth and stresses relief occurring during the sintering operation. It is recommended, therefore, to employ alternative densification methods such as spark plasma sintering or simultaneous pressingsintering operations with the aim of improving the texture of the ceramics. 


\section{REFERENCES}

[1] Panwar, N.L., S.C. Kaushik, and S. Kothari," Role of renewable energy sources in environmental protection: A review", Renewable \& Sustainable Energy Reviews Vol. 15, No. 3, 2011.

[2] Funahashi, R., and S. Urata," Fabrication and application of an oxide thermoelectric system", International Journal of Applied Ceramic Technology Vol. 4, No. 4, 2007.

[3] Riffat, S.B., and X.L. Ma," Thermoelectrics: a review of present and potential applications", Applied Thermal Engineering Vol. 23, No. 8, 2003.

[4] Ohtaki, M.," Recent aspects of oxide thermoelectric materials for power generation from mid-to-high temperature heat source", Journal of the Ceramic Society of Japan Vol. 119, No. 1395, 2011.

[5] Sorrell, C.C., J. Nowotny, S. Sugihara, and ebrary Inc., "Materials for Energy Conversion Devices", Sawston: Woodhead Publishing Limited, 2005.

[6] Shikano, M., and R. Funahashi," Electrical and thermal properties of single-crystalline (Ca2CoO3)(0.7)CoO2 with a Ca3Co4O9 structure", Applied Physics Letters Vol. 82, No. $12,2003$.

[7] Prevel, M., E.S. Reddy, O. Perez, W. Kobayashi, I. Terasaki, C. Goupil, and J.G. Noudem," Thermoelectric properties of sintered and textured Nd-substituted Ca3CO4O9 ceramics", Japanese Journal of Applied Physics Part 1-Regular Papers Brief Communications \& Review Papers Vol. 46, No. 10A, 2007.

[8] Wang, Y., L. Xu, Y. Sui, X. Wang, J. Cheng, and W. Su," Enhanced electron correlation in rare-earth doped Ca3Co4O9", Applied Physics Letters Vol. 97, No. 6, 2010. 
[9] Song, X., Y. Chen, S. Chen, E. Barbero, E.L. Thomas, and P. Barnes," Significant enhancement of electrical transport properties of thermoelectric Ca3Co4O9+delta through Yb doping", Solid State Communications Vol. 152, No. 16, 2012.

[10] Liu, H.Q., X.B. Zhao, T.J. Zhu, Y. Song, and F.P. Wang," Thermoelectric properties of Gd, Y co-doped Ca(3)Co(4)O(9+delta)", Current Applied Physics Vol. 9, No. 2, 2009.

[11] Liu, C.-J., L.-C. Huang, and J.-S. Wang," Improvement of the thermoelectric characteristics of Fe-doped misfit-layered Ca3Co4-xFexO9+delta $(\mathrm{x}=0,0.05,0.1$, and 0.2)", Applied Physics Letters Vol. 89, No. 20, 2006.

[12] Nong, N.V., C.-J. Liu, and M. Ohtaki," High-temperature thermoelectric properties of late rare earth-doped Ca3Co4O9+delta", Journal of Alloys and Compounds Vol. 509, No. 3, 2011.

[13] Wang, Y., Y. Sui, X. Wang, W. Su, and X. Liu," Enhanced high temperature thermoelectric characteristics of transition metals doped Ca3Co4O9+delta by cold highpressure fabrication", Journal of Applied Physics Vol. 107, No. 3, 2010.

[14] Delorme, F., C.F. Martin, P. Marudhachalam, D.O. Ovono, and G. Guzman," Effect of Ca substitution by $\mathrm{Sr}$ on the thermoelectric properties of Ca3Co4O9 ceramics", Journal of Alloys and Compounds Vol. 509, No. 5, 2011.

[15] Wang, Y., Y. Sui, J. Cheng, X. Wang, W. Su, and H. Fan," Influence of Y3+ doping on the high-temperature transport mechanism and thermoelectric response of misfit-layered Ca3Co4O9", Applied Physics a-Materials Science \& Processing Vol. 99, No. 2, 2010.

[16] Sun, T., H.H. Hng, Q.Y. Yan, and J. Ma," Effect of Ag-doping on crystal structure and high temperature thermoelectric properties of c-axis oriented Ca3Co4O9 thin films by pulsed laser deposition", Journal of Alloys and Compounds Vol. 511, No. 1, 2012. 
[17] Wang, Y., Y. Sui, P. Ren, L. Wang, X. Wang, W. Su, and H. Fan," Strongly Correlated Properties and Enhanced Thermoelectric Response in $\mathrm{Ca} 3 \mathrm{CO} 4-\mathrm{xMxO} 9(\mathrm{M}=\mathrm{Fe}, \mathrm{Mn}$, and Cu)", Chemistry of Materials Vol. 22, No. 3, 2010.

[18] Wang, Y., Y. Sui, J. Cheng, X. Wang, and W. Su," Comparison of the high temperature thermoelectric properties for Ag-doped and Ag-added $\mathrm{Ca}(3) \mathrm{Co}(4) \mathrm{O}(9) "$, Journal of Alloys and Compounds Vol. 477, No. 1-2, 2009.

[19] Liu, H.Q., Y. Song, S.N. Zhang, X.B. Zhao, and F.R. Wang," Thermoelectric properties of Ca3-xYxCo4O9+delta ceramics", Journal of Physics and Chemistry of Solids Vol. 70, No. 3-4, 2009.

[20] Pinitsoontorn, S., N. Lerssongkram, N. Keawprak, and V. Amornkitbamrung," Thermoelectric properties of transition metals-doped Ca3Co3.8M0.2O9+delta $(\mathrm{M}=\mathrm{Co}$, $\mathrm{Cr}, \mathrm{Fe}, \mathrm{Ni}, \mathrm{Cu}$ and $\mathrm{Zn}) "$, Journal of Materials Science-Materials in Electronics Vol. 23, No. 5, 2012.

[21] Mikami, M., N. Ando, and R. Funahashi," The effect of Ag addition on electrical properties of the thermoelectric compound Ca3Co4O9", Journal of Solid State Chemistry Vol. 178, No. 7, 2005.

[22] Nong, N.V., N. Pryds, S. Linderoth, and M. Ohtaki," Enhancement of the Thermoelectric Performance of p-Type Layered Oxide Ca3Co4O9+delta Through Heavy Doping and Metallic Nanoinclusions", Advanced Materials Vol. 23, No. 21, 2011, pp. 2484-+.

[23] Xiang, P.-H., Y. Kinernuchi, H. Kaga, and K. Watari," Fabrication and thermoelectric properties of Ca3Co4O9/Ag composites", Journal of Alloys and Compounds Vol. 454, No. 1-2, 2008. 
[24] Song, Y., Q. Sun, L. Zhao, F. Wang, and Z. Jiang," Synthesis and thermoelectric power factor of (Ca0.95Bi0.05)(3)Co4O9/Ag composites", Materials Chemistry and Physics Vol. 113, No. 2-3, 2009.

[25] Faleev, S.V., and F. Leonard," Theory of enhancement of thermoelectric properties of materials with nanoinclusions", Physical Review B Vol. 77, No. 21, 2008.

[26] Kim, W., J. Zide, A. Gossard, D. Klenov, S. Stemmer, A. Shakouri, and A. Majumdar," Thermal conductivity reduction and thermoelectric figure of merit increase by embedding nanoparticles in crystalline semiconductors", Physical Review Letters Vol. 96, No. 4, 2006.

[27] Noudem, J.G.," A new process for lamellar texturing of thermoelectric Ca3Co4O9 oxides by spark plasma sintering", Journal of the European Ceramic Society Vol. 29, No. 12, 2009.

[28] Noudem, J.G., M. Prevel, A. Veres, D. Chateigner, and J. Galy," Thermoelectric Ca3Co4O9 ceramics consolidated by Spark Plasma sintering", Journal of Electroceramics Vol. 22, No. 1-3, 2009.

[29] Noudem, J.G., D. Kenfaui, D. Chateigner, and M. Gomina," Toward the enhancement of thermoelectric properties of lamellar $\mathrm{Ca} 3 \mathrm{Co} 4 \mathrm{O} 9$ by edge-free spark plasma texturing", Scripta Materialia Vol. 66, No. 5, 2012.

[30] Kenfaui, D., G. Bonnefont, D. Chateigner, G. Fantozzi, M. Gomina, and J.G. Noudem," Ca3Co4O9 ceramics consolidated by SPS process: Optimisation of mechanical and thermoelectric properties", Materials Research Bulletin Vol. 45, No. 9, 2010. 
[31] Kenfaui, D., D. Chateigner, M. Gomina, and J.G. Noudem," Texture, mechanical and thermoelectric properties of $\mathrm{Ca} 3 \mathrm{Co} 4 \mathrm{O} 9$ ceramics", Journal of Alloys and Compounds Vol. 490, No. 1-2, 2010.

[32] Kenfaui, D., B. Lenoir, D. Chateigner, B. Ouladdiaf, M. Gomina, and J.G. Noudem," Development of multilayer textured $\mathrm{Ca} 3 \mathrm{Co} 4 \mathrm{O} 9$ materials for thermoelectric generators: Influence of the anisotropy on the transport properties", Journal of the European Ceramic Society Vol. 32, No. 10, 2012.

[33] Mikami, M., E. Guilmeau, R. Funahashi, K.J. Chong, and D. Chateigner," Enhancement of electrical properties of the thermoelectric compound $\mathrm{Ca} 3 \mathrm{Co} 4 \mathrm{O} 9$ through use of largegrained powder", Journal of Materials Research Vol. 20, No. 9, 2005.

[34] Guilmeau, E., R. Funahashi, M. Mikami, K. Chong, and D. Chateigner," Thermoelectric properties-texture relationship in highly oriented Ca3Co4O9 composites", Applied Physics Letters Vol. 85, No. 9, 2004.

[35] Prevel, M., S. Lemonnier, Y. Klein, S. Hebert, D. Chateigner, B. Ouladdiaf, and J.G. Noudem," Textured $\mathrm{Ca} 3 \mathrm{Co} 4 \mathrm{O} 9$ thermoelectric oxides by thermoforging process", Journal of Applied Physics Vol. 98, No. 9, 2005.

[36] François, L., and G. Eric, "Temperature Measurements", Thermal Measurements and Inverse Techniques: CRC Press, 2011, pp. 95-142.

[37] Yakabe, H., K. Kikuchi, I. Terasaki, Y. Sasago, K. Uchinokura, and Ieee," Thermoelectric properties of transition-metal oxide $\mathrm{NaCo} 2 \mathrm{O} 4$ system", Proceedings Ict'97 - Xvi International Conference on Thermoelectrics, 1997.

[38] Terasaki, I., Y. Sasago, and K. Uchinokura," Large thermoelectric power in NaCo2O4 single crystals", Physical Review B Vol. 56, No. 20, 1997. 
[39] Sotelo, A., G. Constantinescu, S. Rasekh, M.A. Torres, J.C. Diez, and M.A. Madre," Improvement of thermoelectric properties of $\mathrm{Ca} 3 \mathrm{Co} 4 \mathrm{O} 9$ using soft chemistry synthetic methods", Journal of the European Ceramic Society Vol. 32, No. 10, 2012.

[40] Interrante, L.V., and M.J. Hampden-Smith, Chemistry of advanced materials : an overview, New York: Wiley-VCH, 1998.

[41] Sakka, S., Handbook of sol-gel science and technology : processing, characterization, and applications, Boston: Kluwer Academic Publishers, 2005.

[42] Schwartz, M.M., "Smart materials", Boca Raton: CRC Press, 2009.

[43] Lee, B.I., and S. Komarneni, "Chemical processing of ceramics", Materials engineering 27, Boca Raton: Taylor \& Francis, 2005.

[44] Groza, J.R., "Materials processing handbook", Boca Raton, FL: CRC Press, 2007.

[45] Munir, Z.A., U. Anselmi-Tamburini, and M. Ohyanagi," The effect of electric field and pressure on the synthesis and consolidation of materials: A review of the spark plasma sintering method", Journal of Materials Science Vol. 41, No. 3, 2006.

[46] Zhou, Y.Q., I. Matsubara, S. Horii, T. Takeuchi, R. Funahashi, M. Shikano, J. Shimoyama, K. Kishio, W. Shin, N. Izu, and N. Murayama," Thermoelectric properties of highly grain-aligned and densified Co-based oxide ceramics", Journal of Applied Physics Vol. 93, No. 5, 2003.

[47] Zanella, R., S. Giorgio, C.R. Henry, and C. Louis," Alternative methods for the preparation of gold nanoparticles supported on TiO2", Journal of Physical Chemistry B Vol. 106, No. 31, 2002. 
[48] Delannoy, L., N. El Hassan, A. Musi, N.N. Le To, J.-M. Krafft, and C. Louis," Preparation of supported gold nanoparticles by a modified incipient wetness impregnation method", Journal of Physical Chemistry B Vol. 110, No. 45, 2006. 